\title{
The Journal of Organic Chemistry
}

Subscriber access provided by The Bodleian Libraries of The University of Oxford

\section{Article}

\section{Asymmetric Synthesis of the Tetraponerine Alkaloids}

Stephen G. Davies, Ai M. Fletcher, Ian T. T. Houlsby, Paul M. Roberts, and James E. Thomson

J. Org. Chem., Just Accepted Manuscript • DOI: 10.1021/acs.joc.7b00837 • Publication Date (Web): 24 May 2017

Downloaded from http://pubs.acs.org on June 6, 2017

\section{Just Accepted}

"Just Accepted" manuscripts have been peer-reviewed and accepted for publication. They are posted online prior to technical editing, formatting for publication and author proofing. The American Chemical Society provides "Just Accepted" as a free service to the research community to expedite the dissemination of scientific material as soon as possible after acceptance. "Just Accepted" manuscripts appear in full in PDF format accompanied by an HTML abstract. "Just Accepted" manuscripts have been fully peer reviewed, but should not be considered the official version of record. They are accessible to all readers and citable by the Digital Object Identifier (DOI®). "Just Accepted" is an optional service offered to authors. Therefore, the "Just Accepted" Web site may not include all articles that will be published in the journal. After a manuscript is technically edited and formatted, it will be removed from the "Just Accepted" Web site and published as an ASAP article. Note that technical editing may introduce minor changes to the manuscript text and/or graphics which could affect content, and all legal disclaimers and ethical guidelines that apply to the journal pertain. ACS cannot be held responsible for errors or consequences arising from the use of information contained in these "Just Accepted" manuscripts. 


\title{
Asymmetric Synthesis of the Tetraponerine Alkaloids
}

\author{
Stephen G. Davies, * Ai M. Fletcher, Ian T. T. Houlsby \\ Paul M. Roberts, and James E. Thomson \\ Department of Chemistry, Chemistry Research Laboratory, University of Oxford, \\ Mansfield Road, Oxford, OX1 3TA, U.K. \\ steve.davies@chem.ox.ac.uk
}
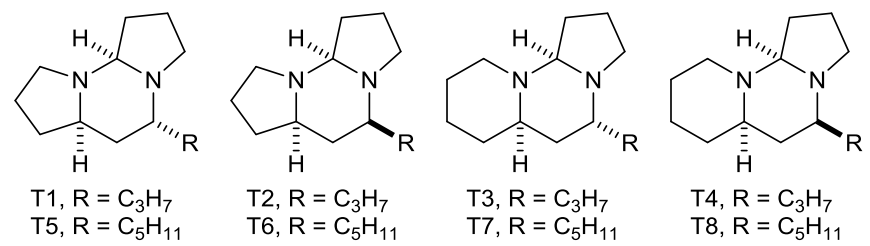

The asymmetric syntheses of all eight tetraponerine alkaloids (T1-T8) were achieved using the diastereoselective conjugate additions of lithium amide reagents in the key stereodefining steps. Conjugate addition of either lithium $(R)-N$-allyl- $N$-( $\alpha$-methylbenzyl)amide or lithium $(R)-N$-(but-3-en-1-yl)- $N$ - $(\alpha$ methylbenzyl)amide to tert-butyl sorbate was followed by ring-closing metathesis of the resultant $N$-alkenyl $\beta$-amino esters, reduction to the corresponding aldehydes, and reaction with tert-butyl (triphenylphosphoranylidene)acetate. Subsequent conjugate addition of the requisite antipode of lithium $N$ benzyl- $N$-( $\alpha$-methylbenzyl)amide to the resultant $\alpha, \beta$-unsaturated esters gave a range of diamines for elaboration to $\mathrm{T} 1-\mathrm{T} 8$ via a sequence involving reduction of the ester moiety to give the corresponding aldehyde, olefination, tandem hydrogenation/hydrogenolysis, and cyclisation upon reaction with 4bromobutanal to give the tricyclic skeleton.

\section{Introduction}

Tetraponerines 1-8 (which are also known as T1-T8, respectively) are a series of tricyclic aminals first isolated from the paralyzing venom of the New Guinean pseudomyrmecine ant Tetraponera $s p .{ }^{1}$ These alkaloids represent the major constituents of the contact poison secreted by host ants as a defence against enemy colonies: when smeared with the contact poison enemies display signs of paralysis and ultimately death, presumably by inhibition of nicotinic acetylcholine receptors. ${ }^{2}$ The structures of the tetraponerine alkaloids are based on either a decahydrodipyrrolo[1,2-a:1',2'-c]pyrimidine skeleton 1 (for tetraponerines 1 , 2, 5 and 6) or a decahydro-5H-pyrido[1,2-c]pyrrolo[1,2-a]pyrimidine skeleton 2 (for tetraponerines 3, 4, 7 
and 8), with a $\mathrm{C}(5)$-alkyl substituent being present in all cases. Within each heterocyclic series, the alkaloids differ in their configurations at $\mathrm{C}(5)$ and also in the identity of the $\mathrm{C}(5)$-alkyl substituent (Fig. 1). (+)Tetraponerine 8 is the only member of this family for which an X-ray crystal structure has been obtained to confirm both its gross structure and relative configuration. ${ }^{1}$ The absolute configurations of tetraponerines $3-$ 8 have been established by separate chemical syntheses and comparison of the specific rotations for the natural and synthetic material. ${ }^{3-5}$ Synthetic samples of (+)-tetraponerine 1 and (+)-tetraponerine 2 have also been prepared, ${ }^{5,6}$ although the absolute configurations of the natural materials have not been unambiguously established as only very small amounts were obtained from natural sources. ${ }^{4,7}$ The unusual tricyclic aminal structure of the tetraponerine alkaloids, as well as their potent insecticidal activity, has meant that they have become popular targets for total synthesis. Since their isolation in 1987, there have been several syntheses of these alkaloids in both racemic ${ }^{8}$ and enantiopure ${ }^{3-6,9}$ form. ${ }^{10,11}$ Herein, we report total syntheses of all eight tetraponerine alkaloids using our diastereoselective lithium amide conjugate addition methodology ${ }^{12}$ in the key stereodefining steps.

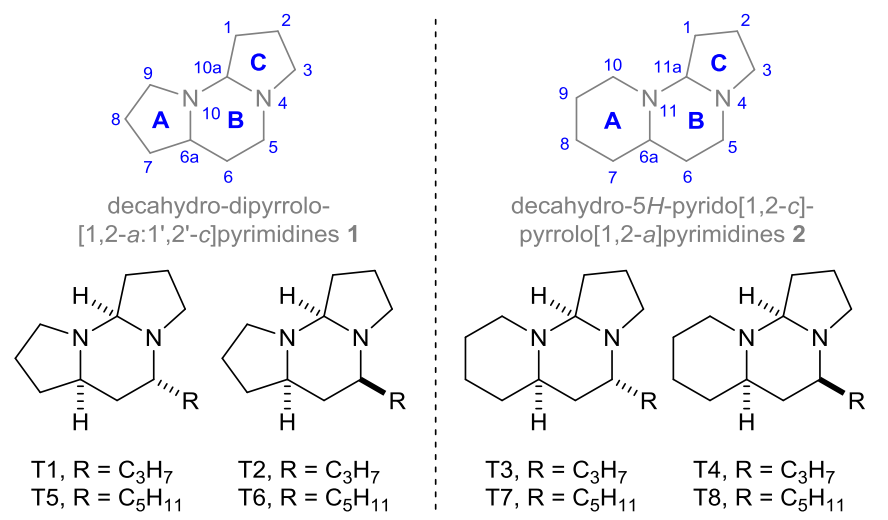

FIGURE 1. The structures of the tetraponerine alkaloids T1-T8.

Our divergent synthetic strategy towards the tetraponerine alkaloids involved two iterative lithium amide conjugate additions to install the requisite stereochemistry. In the first instance, conjugate addition of either lithium $(R)-N$-allyl- $N$-( $(\alpha$-methylbenzyl)amide $(R)-4(\mathrm{n}=1)$ or lithium $(R)-N$-(but-3-en-1-yl)- $N-(\alpha-$ methylbenzyl)amide $(R)-\mathbf{5}(\mathrm{n}=2)$ to tert-butyl sorbate $3,{ }^{13-15}$ followed by ring-closing metathesis of the resultant $N$-alkenyl $\beta$-amino ester $6(\mathrm{n}=1$ or 2$)$ and homologation would give the key $\alpha, \beta$-unsaturated ester 7 ( $\mathrm{n}=1$ or 2$)$. Subsequent conjugate addition of the requisite antipode of lithium $N$-benzyl- $N-(\alpha-$ methylbenzyl)amide 8 to $\alpha, \beta$-unsaturated ester $7(n=1$ or 2$)$ would then provide a range of diamines 9 and 10 ( $\mathrm{n}=1$ or 2 in both cases) for elaboration. In each case, a sequence involving reduction of the ester moiety to the corresponding aldehyde, olefination, and tandem hydrogenation/hydrogenolysis would produce the corresponding diamines 11 or 12 ( $\mathrm{n}=1$ or 2 in both cases), which have previously been converted into the 
tetraponerine alkaloids by Gonzalez-Gomez et al. ${ }^{6,9 \mathrm{~d}}$ upon reaction with 4-bromobutanal (Fig. 2). As part of their study, Gonzalez-Gomez et al. proposed that the configuration at the aminal functionality within each of the tetraponerine alkaloids is susceptible to equilibration via the corresponding iminium ion and that the natural products may therefore possess the most thermodynamically favourable relative configurations with respect to epimerisation at the aminal centre.

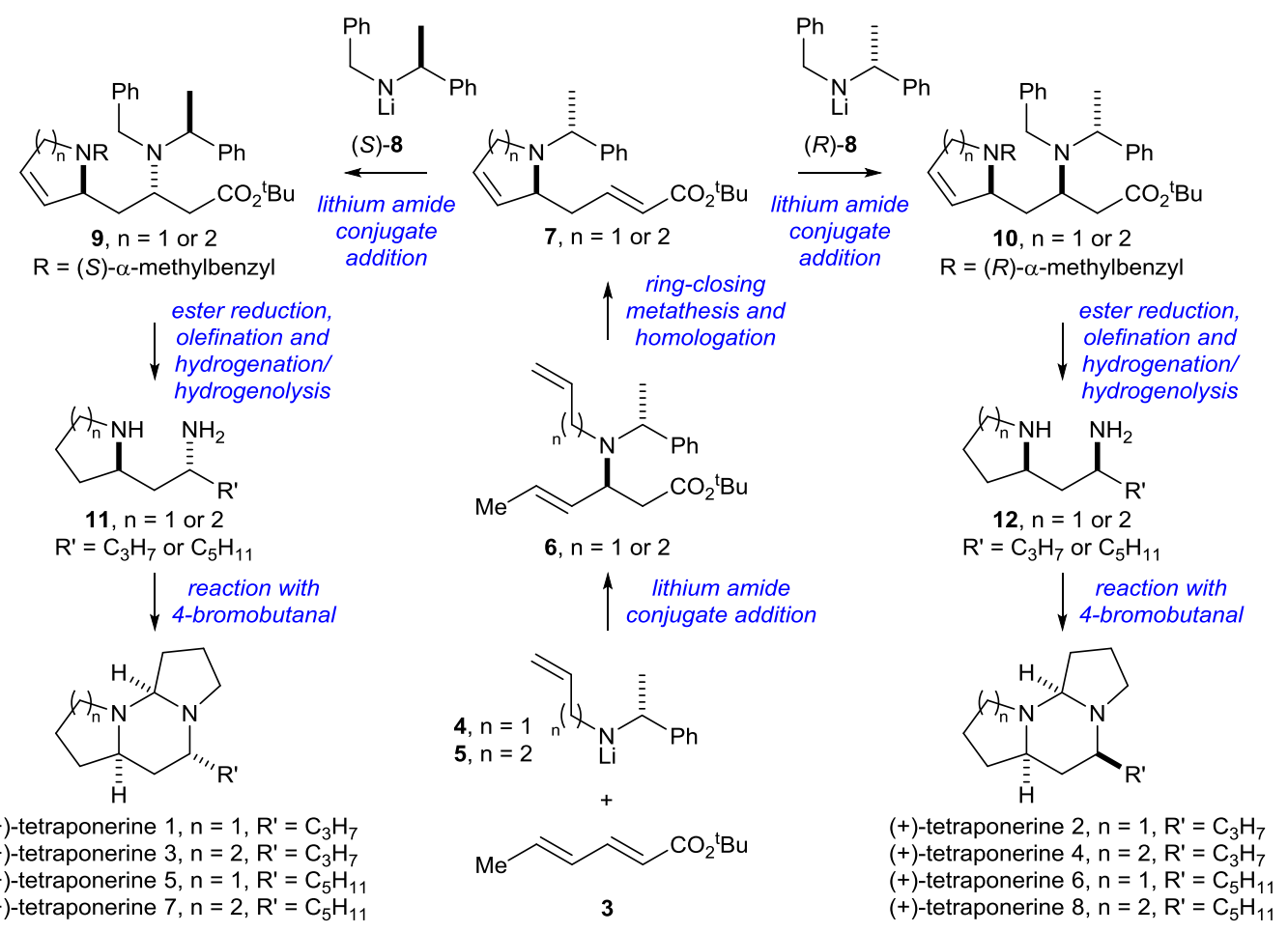

FIGURE 2. Proposed synthetic strategy to access all eight tetraponerine alkaloids.

\section{Results and Discussion}

The tetraponerine alkaloids based upon a decahydro-5H-pyrido[1,2-c]pyrrolo[1,2- $a$ ]pyrimidine skeleton 2 (i.e., tetraponerines 3, 4, 7 and 8) were targeted initially. Tetrahydropyridine $\mathbf{1 4}$ was prepared via a known sequence of reactions, ${ }^{13,14}$ involving conjugate addition of lithium $(R)-N$-(but-3-en-1-yl)- $N-(\alpha$ methylbenzyl)amide $(R)-\mathbf{5}$ to tert-butyl sorbate $3,{ }^{16}$ which gave $\beta$-amino ester $\mathbf{1 3}$ as a single diastereoisomer (>99:1 dr) in 69\% yield. Subsequent ring-closing metathesis of $\mathbf{1 3}$ gave tetrahydropyridine $\mathbf{1 4}$ in $83 \%$ yield. ${ }^{13,14}$ A superior overall yield of $\mathbf{1 4}$ from tert-butyl sorbate $\mathbf{3}$ was obtained by omitting the purification of the intermediate $\beta$-amino ester 13; following this sequence, tetrahydropyridine 14 was isolated in $73 \%$ yield (from 3) and >99:1 dr. Reduction of $\mathbf{1 4}$ with $\mathrm{LiAlH}_{4}$ gave the corresponding alcohol $\mathbf{1 5}$ in $94 \%$ yield as a single diastereoisomer. Swern oxidation of primary alcohol $\mathbf{1 5}$ followed by Wittig reaction of the resultant aldehyde with $\mathrm{Ph}_{3} \mathrm{P}=\mathrm{CHCO}_{2}{ }^{\mathrm{t}} \mathrm{Bu}$ gave a 93:7 mixture of diastereoisomeric products $\mathbf{1 6}$ and $\mathbf{1 7}$, respectively. Purification of the crude reaction mixture gave the major product 16 in $46 \%$ yield and $>99: 1 \mathrm{dr}[(E):(Z)]$, and the minor product $\mathbf{1 7}$ in 3\% yield and >99:1 dr [(Z):(E)]. However, a superior yield of $\mathbf{1 6}$ was obtained from 
the reduction of $\beta$-amino ester 14 with DIBAL-H followed by in situ Wittig reaction of the intermediate aldehyde with $\mathrm{Ph}_{3} \mathrm{P}=\mathrm{CHCO}_{2}{ }^{\mathrm{t}} \mathrm{Bu}$, which gave an 80:20 mixture of $\mathbf{1 6}$ and $\mathbf{1 7}$, respectively, from which $\mathbf{1 6}$ was isolated in $72 \%$ yield and >99:1 dr $[(E):(Z)]$, and 17 was isolated in 14\% yield and >99:1 $\mathrm{dr}[(Z):(E)]$ (Scheme 1).

\section{SCHEME 1. Preparation of tetrahydropyridine 16}

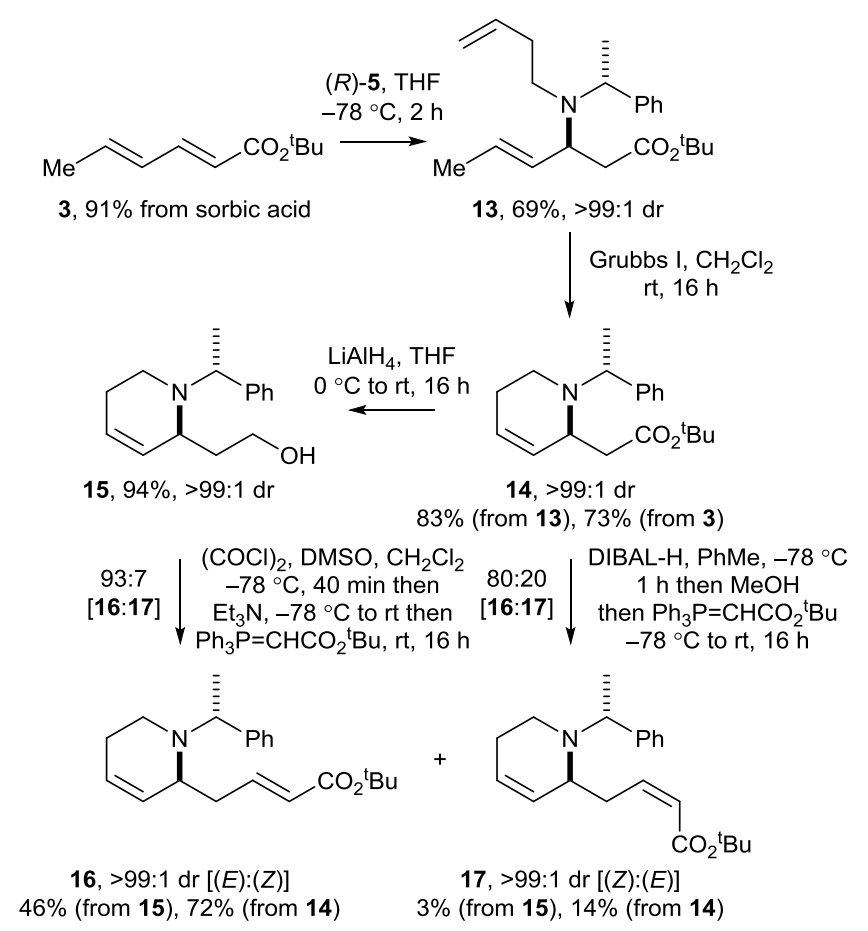

The inherent level of substrate control offered by chiral $\alpha, \beta$-unsaturated ester $\mathbf{1 6}$ upon reaction with a lithium amide reagent was probed upon conjugate addition of lithium $N$-isopropyl- $N$-benzylamide 18, which we have previously employed as an achiral model for the conjugate addition of either antipode of enantiopure lithium $N$-benzyl- $N$-( $\alpha$-methylbenzyl)amide $8 .^{17}$ Reaction of $\mathbf{1 6}$ with the achiral lithium amide reagent $\mathbf{1 8}$ gave a 50:50 mixture of the diastereoisomeric $\beta$-amino ester adducts $\mathbf{1 9}$ and $\mathbf{2 0}$, which were isolated in $96 \%$ combined yield. This result suggests that $\alpha, \beta$-unsaturated ester 16 elicits essentially no diastereoselectivity in the conjugate addition reaction, and is consistent with the low levels of substrate control observed upon conjugate addition of lithium amides to substrates bearing remote stereogenic centres. ${ }^{18}$ The conjugate addition of lithium $(R)-N$-benzyl- $N$-( $\alpha$-methylbenzyl)amide $(R)-\mathbf{8}$ to $\alpha, \beta$-unsaturated ester 16 gave $\beta$-amino ester 21 in $>95: 5 \mathrm{dr}$, and conjugate addition of lithium $(S)$ - $N$-benzyl- $N$ - $(\alpha-$ methylbenzyl)amide $(S)-\mathbf{8}$ to $\mathbf{1 6}$ gave $\beta$-amino ester 22 in >95:5 dr. Following purification of the crude reaction mixtures, 21 was isolated in 97\% yield and >99:1 dr, and 22 was isolated in 94\% yield and >99:1 $\mathrm{dr}$ (Scheme 2). The configurations of the newly formed stereogenic centres within 21 and 22 were assigned on the assumption that conjugate addition proceeds under the dominant stereocontrol of the lithium amide 
reagent 8 (given the total lack of substrate control observed upon conjugate addition of lithium $N$-isopropyl$N$-benzylamide 18 to $\alpha, \beta$-unsaturated ester 16), and is consistent with the formation of 21 and 22 as single diastereoisomers in both cases. These stereochemical assignments were later confirmed upon derivatisation to the target tetraponerine alkaloids.

\section{SCHEME 2. Conjugate additions to 16}
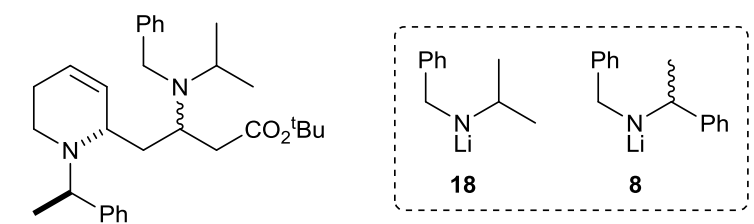

19/20, 96\%, 50:50 dr

$\begin{gathered}\text { 18, THF } \\ -78{ }^{\circ} \mathrm{C}, 2 \mathrm{~h}\end{gathered} \mid 50: 50 \mathrm{dr}$

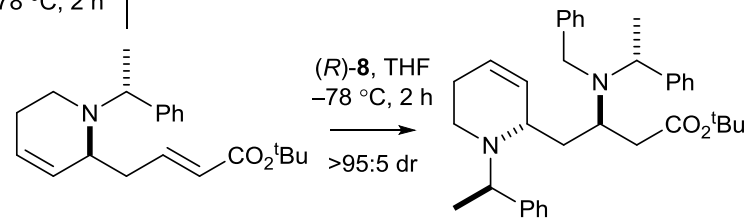

16, >99:1 dr [(E):(Z)]

21, $97 \%,>99: 1 d r$

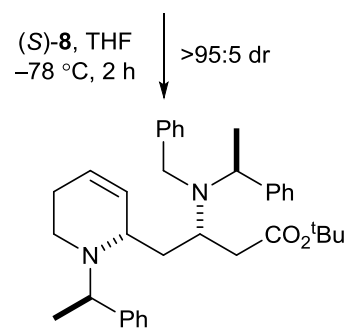

22, $94 \%,>99: 1 d r$

The elaboration of 21 to (+)-tetraponerine 4 (27) was achieved via reduction of the ester moiety within 21 with DIBAL-H followed by Wittig reaction of the resultant aldehyde with $\mathrm{Ph}_{3} \mathrm{P}=\mathrm{CH}_{2}$, which gave olefin 23 in $62 \%$ and >99:1 dr. Tandem Pd-mediated hydrogenation/hydrogenolysis of $\mathbf{2 3}$ in a mixture of $\mathrm{MeOH}, \mathrm{H}_{2} \mathrm{O}$ and $\mathrm{AcOH}$ gave diamine 25 in 73\% isolated yield and >99:1 dr. Finally, reaction of 25 with 4bromobutanal and $\mathrm{K}_{2} \mathrm{CO}_{3}$ in $\mathrm{CH}_{2} \mathrm{Cl}_{2}$, according to the literature protocol, ${ }^{6,9 \mathrm{~d}}$ gave (+)-tetraponerine 4 (27) in $68 \%$ yield and >99:1 dr. The spectroscopic data and specific rotation for this sample of (+)-tetraponerine 4 (27) were in good agreement with literature values $\left\{[\alpha]_{\mathrm{D}}^{25}+90.8\left(c 1.0\right.\right.$ in $\left.\mathrm{CHCl}_{3}\right)$; lit. ${ }^{4}$ for a sample isolated from the natural source $[\alpha]_{\mathrm{D}}^{20}+94\left(c 0.2\right.$ in $\left.\mathrm{CHCl}_{3}\right)$; lit. ${ }^{9 \mathrm{~b}}[\alpha]_{\mathrm{D}}^{20}+96\left(c 2.0\right.$ in $\left.\left.\mathrm{CHCl}_{3}\right)\right\}$, thereby confirming the assigned configurations of the synthetic precursors 21, 23 and $\mathbf{2 5}$. Repetition of this hydrogenation/hydrogenolysis and ring-closure reaction sequence, but omitting the purification of the intermediate diamine 25, gave (+)-tetraponerine 4 (27) in 54\% overall yield (from 23), representing a marginal improvement over the stepwise protocol (i.e., $50 \%$ overall yield from $\mathbf{2 3})$. (+)-Tetraponerine $8(\mathbf{2 8})$ was also prepared from 21 in a directly analogous manner: one-pot reduction/Wittig reaction of 21 gave 
olefin 24 in $62 \%$ yield and >95:5 dr $[(Z):(E)] .{ }^{19}$ Subsequent tandem hydrogenation/hydrogenolysis of 24 gave diamine $\mathbf{2 6}$ (although in this case only it was necessary to re-subject the hydrogenation/hydrogenolysis reaction using $\mathrm{Pd}(\mathrm{OH})_{2}$ as the catalyst to effect complete $N$-debenzylation). Diamine 26 was then immediately converted into (+)-tetraponerine 8 (28) upon treatment with 4-bromobutanal. ${ }^{6,9 d}$ Following purification of the crude reaction mixture, (+)-tetraponerine 8 (28) was isolated as a single diastereoisomer (>99:1 dr) in 67\% yield from 24 (Scheme 3). The spectroscopic data, melting point and specific rotation for this sample of (+)-tetraponerine 8 (28) were all in good agreement with literature values $\left\{\mathrm{mp} 38-40{ }^{\circ} \mathrm{C}\right.$; lit. ${ }^{5}$ mp $40{ }^{\circ} \mathrm{C} ;[\alpha]_{\mathrm{D}}^{25}+93.0\left(c 1.0\right.$ in $\left.\mathrm{CHCl}_{3}\right)$; lit. ${ }^{4}$ for a sample isolated from the natural source $[\alpha]_{\mathrm{D}}^{20}+102(c 0.2$ in $\mathrm{CHCl}_{3}$ ); lit. $^{9 \mathrm{~b}}[\alpha]_{\mathrm{D}}^{20}+101$ ( $c 2.0$ in $\left.\left.\mathrm{CHCl}_{3}\right)\right\}$, thereby confirming the assigned configurations of the synthetic precursors 21, 24 and 26. The asymmetric syntheses of (+)-tetraponerine 4 (27) and (+)tetraponerine 8 (28) were therefore achieved in 15.5 and $19.3 \%$ overall yield, respectively, in eight steps from commercially available starting materials.

\section{SCHEME 3. Preparation of (+)-tetraponerine 4 and (+)-tetraponerine 8}

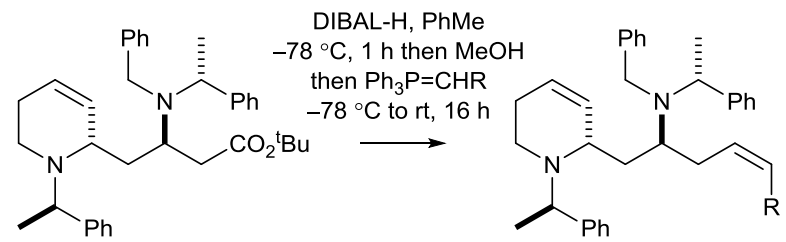

21, $>99: 1 \mathrm{dr} \quad$ 23, $\mathrm{R}=\mathrm{H}, 62 \%,>99: 1 \mathrm{dr}$

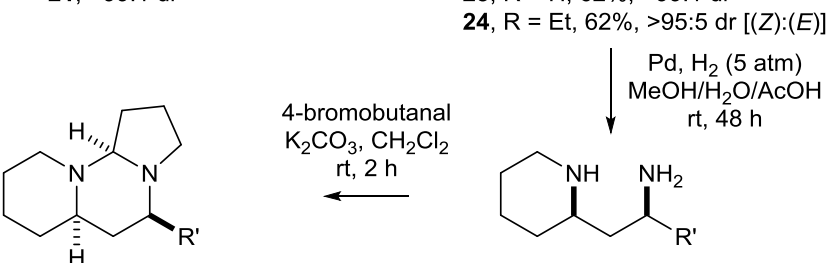

27, $\mathrm{R}=\mathrm{C}_{3} \mathrm{H}_{7},(+)$-tetraponerine 4 $68 \%$ (from 25), $>99: 1 \mathrm{dr}$ $54 \%$ (from 23), >99:1 dr

25, $\mathrm{R}^{\prime}=\mathrm{C}_{3} \mathrm{H}_{7}, 73 \%,>99: 1 \mathrm{dr}$

$\mathrm{R}=\mathrm{C}_{5} \mathrm{H}_{11},(+)$-tetraponerine 8 26, $\mathrm{R}^{\prime}=\mathrm{C}_{5} \mathrm{H}_{11}$, not isolated

$67 \%$ (from 24), >99:1 dr

Elaboration of the diastereoisomeric substrate 22 to (+)-tetraponerine $3(\mathbf{3 3})$ and (+)-tetraponerine 7 (34) was also evaluated. Reduction of 22 with DIBAL-H followed by Wittig reaction of the resultant aldehyde with the requisite ylid reagent $\mathrm{Ph}_{3} \mathrm{P}=\mathrm{CHR}$ gave either $29(\mathrm{R}=\mathrm{H})$ or $30(\mathrm{R}=\mathrm{Et})$; after chromatographic purification of the crude reaction mixtures, 29 was isolated in $54 \%$ yield as a single diastereoisomer and $\mathbf{3 0}$ was isolated in $47 \%$ yield and $>95: 5 \mathrm{dr} \quad[(Z):(E)] .^{20}$ Tandem hydrogenation/hydrogenolysis of $\mathbf{2 9}$ and $\mathbf{3 0}$ gave the corresponding diamines $\mathbf{3 1}$ and $\mathbf{3 2}$, which were immediately reacted with 4-bromobutanal ${ }^{6,9 \mathrm{~d}}$ to give (+)-tetraponerine $3(\mathbf{3 3})$ and (+)-tetraponerine $7(\mathbf{3 4})$ in 60 and 44\% yield, respectively, as single diastereoisomers (>99:1 dr) in each case (Scheme 4). The 
spectroscopic data and specific rotations for these samples of (+)-tetraponerine $3(\mathbf{3 3})$ and (+)-tetraponerine 7 (34) were in good agreement with literature values $\left\{\right.$ for (+)-tetraponerine $3(\mathbf{3 3}):[\alpha]_{\mathrm{D}}^{25}+30.9(c) 1.0$ in $\left.\mathrm{CHCl}_{3}\right)$; lit. ${ }^{4}$ for a sample isolated from the natural source $[\alpha]_{\mathrm{D}}^{20}+27\left(c 0.07\right.$ in $\left.\mathrm{CHCl}_{3}\right) ;$ lit. ${ }^{5}[\alpha]_{\mathrm{D}}^{20}+31(c 3.1$ in $\left.\mathrm{CHCl}_{3}\right)$; lit. ${ }^{9 \mathrm{~d}}[\alpha]_{\mathrm{D}}^{20}+35\left(c 0.49\right.$ in $\left.\mathrm{CHCl}_{3}\right)$; for (+)-tetraponerine $7(\mathbf{3 4})$ : $[\alpha]_{\mathrm{D}}^{25}+30.4\left(c 0.8\right.$ in $\left.\mathrm{CHCl}_{3}\right)$; lit. $^{4}$ for a sample isolated from the natural source $[\alpha]_{\mathrm{D}}^{20}+30\left(c 0.22\right.$ in $\left.\mathrm{CHCl}_{3}\right) ;$ lit. $^{9 \mathrm{~b}}[\alpha]_{\mathrm{D}}^{20}+29.5\left(c 2.2\right.$ in $\left.\left.\mathrm{CHCl}_{3}\right)\right\}$, thereby confirming the assigned configurations of the synthetic precursors 22 and 29-32. The asymmetric syntheses of (+)-tetraponerine $3(\mathbf{3 3})$ and (+)-tetraponerine $7(\mathbf{3 4})$ were therefore achieved in 14.6 and $9.3 \%$ overall yield, respectively, in eight steps from commercially available starting materials.

\section{SCHEME 4. Preparation of (+)-tetraponerine 3 and (+)-tetraponerine 7}

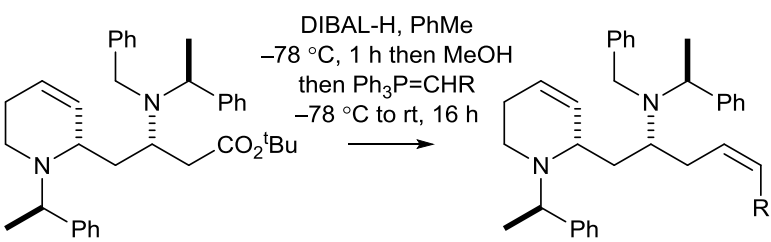

22, $>99: 1 \mathrm{dr}$

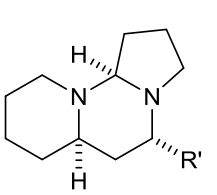

33, $\mathrm{R}^{\prime}=\mathrm{C}_{3} \mathrm{H}_{7},(+)$-tetraponerine 3 $60 \%$ (from 29), $>99: 1 \mathrm{dr}$ 34, $\mathrm{R}^{\prime}=\mathrm{C}_{5} \mathrm{H}_{11},(+)$-tetraponerine 7

$44 \%$ (from 30 ), $>99: 1 \mathrm{dr}$
29, $\mathrm{R}=\mathrm{H}, 54 \%,>99: 1 \mathrm{dr}$ 30, $\mathrm{R}=\mathrm{Et}, 47 \%,>95: 5 \mathrm{dr}[(Z):(E)]$

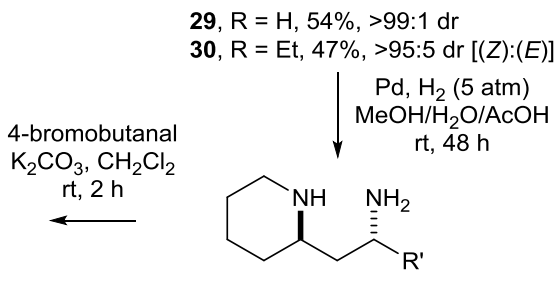

31, $\mathrm{R}^{\prime}=\mathrm{C}_{3} \mathrm{H}_{7}$, not isolated 32, $\mathrm{R}^{\prime}=\mathrm{C}_{5} \mathrm{H}_{11}$, not isolated

The remaining tetraponerine alkaloids in the series, based on a decahydrodipyrrolo[1,2-a:1',2'c]pyrimidine scaffold 1 (i.e., tetraponerines 1, 2, 5 and 6), were targeted next. Dihydropyrrole 36 was prepared via a known sequence of reactions, ${ }^{13,15}$ involving conjugate addition of lithium $(R)-N$-allyl- $N-(\alpha-$ methylbenzyl)amide $(R)-\mathbf{4}$ to tert-butyl sorbate $3,{ }^{16}$ which gave $\beta$-amino ester $\mathbf{3 5}$ in $89 \%$ yield as a single diastereoisomer (>99:1 dr). Subsequent ring-closing metathesis of $\mathbf{3 5}$ gave dihydropyrrole $\mathbf{3 6}$ in $86 \%$ yield and >99:1 dr. ${ }^{13,15}$ In this case, it was found that hydrogenation of $\mathbf{3 6}$ with Wilkinson's catalyst $\left[\mathrm{RhCl}\left(\mathrm{PPh}_{3}\right)_{3}\right]^{13,15 a, b}$ to give pyrrolidine 37 was necessary to avoid oxidative side reactions, leading to various pyrrole containing species, in the subsequent steps. ${ }^{21}$ Reduction of the ester moiety within pyrrolidine 37 with DIBAL-H followed by in situ Wittig reaction of the corresponding aldehyde with $\mathrm{Ph}_{3} \mathrm{P}=\mathrm{CHCO}_{2}{ }^{\mathrm{t}} \mathrm{Bu}$ gave a 75:25 mixture of $\mathbf{3 8}$ and 39, respectively. Following purification of the crude reaction mixture, an 86:14 mixture of $\mathbf{3 8}$ and $\mathbf{3 9}$, respectively, was isolated in $66 \%$ combined yield in addition to a 9:91 mixture of $\mathbf{3 8}$ and $\mathbf{3 9}$ in 13\% combined yield. Upon repetition of the reaction sequence, hydrogenation of dihydropyrrole $\mathbf{3 6}$ followed by immediate reduction of the crude reaction mixture with 
DIBAL-H and Wittig reaction of the resultant aldehyde gave 38 in 50\% yield (from 36) and 98:2 dr (Scheme $5)$.

\section{SCHEME 5. Preparation of pyrrolidine 38}
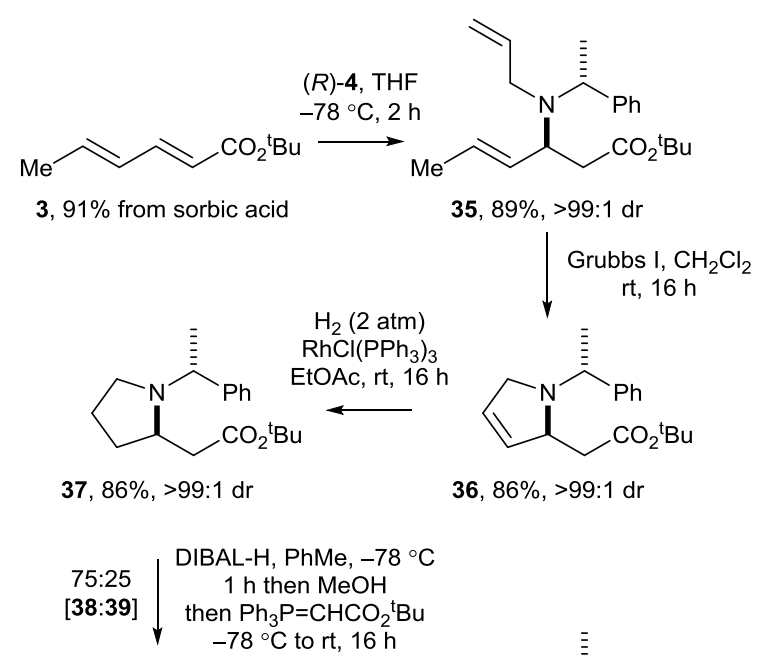

36, $86 \%$, >99:1 dr

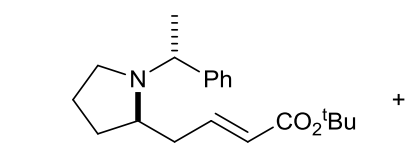

38, $66 \%$ (from 37), 86:14 dr $[(E):(Z)]$ $50 \%$ (from 36), $98: 2 \mathrm{dr}[(E):(Z)]$

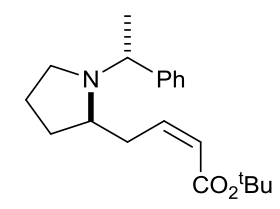

$39,13 \%$ (from 37 ) $9: 91 \mathrm{dr}[(E):(Z)]$

The inherent level of substrate control offered by $\alpha, \beta$-unsaturated ester 38 (98:2 dr [(E):(Z)]) was probed upon conjugate addition of lithium $N$-isopropyl- $N$-benzylamide 18, which gave a 50:50 mixture of the diastereoisomeric $\beta$-amino ester adducts $\mathbf{4 0}$ and $\mathbf{4 1}$. Following purification of the crude reaction mixture, 40 and 41 were isolated as a 50:50 mixture in 70\% combined yield. This result suggests that $\alpha, \beta$-unsaturated ester 38 elicits essentially no diastereoselectivity in the conjugate addition reaction, as was also the case for the homologous tetrahydropyridine containing substrate $\mathbf{1 6}$, and is again consistent with the low levels of substrate control observed upon conjugate addition of lithium amides to other substrates bearing remote stereogenic centres. ${ }^{18}$ The conjugate addition of lithium $(R)-N$-benzyl- $N$ - $(\alpha$-methylbenzyl)amide $(R)-8$ to $\alpha, \beta$-unsaturated ester 38 (98:2 dr $[(E):(Z)])$ gave $\beta$-amino ester 42 in 98\% yield and >99:1 dr, whereas conjugate addition of lithium $(S)-N$-benzyl- $N$-( $\alpha$-methylbenzyl)amide $(S)-\mathbf{8}$ to 38 (98:2 $\mathrm{dr}[(E):(Z)])$ gave $\beta$ amino ester $\mathbf{4 3}$ in $92 \%$ isolated yield and >99:1 dr (Scheme 6). The configurations of the newly formed stereogenic centres within the conjugate addition products 42 and 43 were assigned, as before, on the assumption that conjugate addition proceeds under the dominant stereocontrol of the lithium amide reagent in both cases; these assignments were later confirmed upon derivatisation to the target alkaloids. Conjugate addition of lithium amide $(R)-\mathbf{8}$ to an $86: 14$ mixture of $(E)-38$ and $(Z)-39$, respectively, gave an 86:14 mixture of $\beta$-amino ester $\mathbf{4 2}$ and a species which was tentatively assigned as the corresponding $\beta, \gamma-$ 
unsaturated derivative 44; there was no evidence of either $(E)-38$ or $(Z)-39$ in the ${ }^{1} \mathrm{H}$ NMR spectrum of the crude reaction mixture. As $\beta$-amino ester $\mathbf{4 2}$ and $\beta, \gamma$-unsaturated ester $\mathbf{4 4}$ were only partially separable upon attempted chromatographic purification, this result confirmed that it was necessary to enrich the diastereoisomeric purity of the $\alpha, \beta$-unsaturated ester prior to attempting the conjugate addition reaction.

SCHEME 6. Conjugate additions to 38
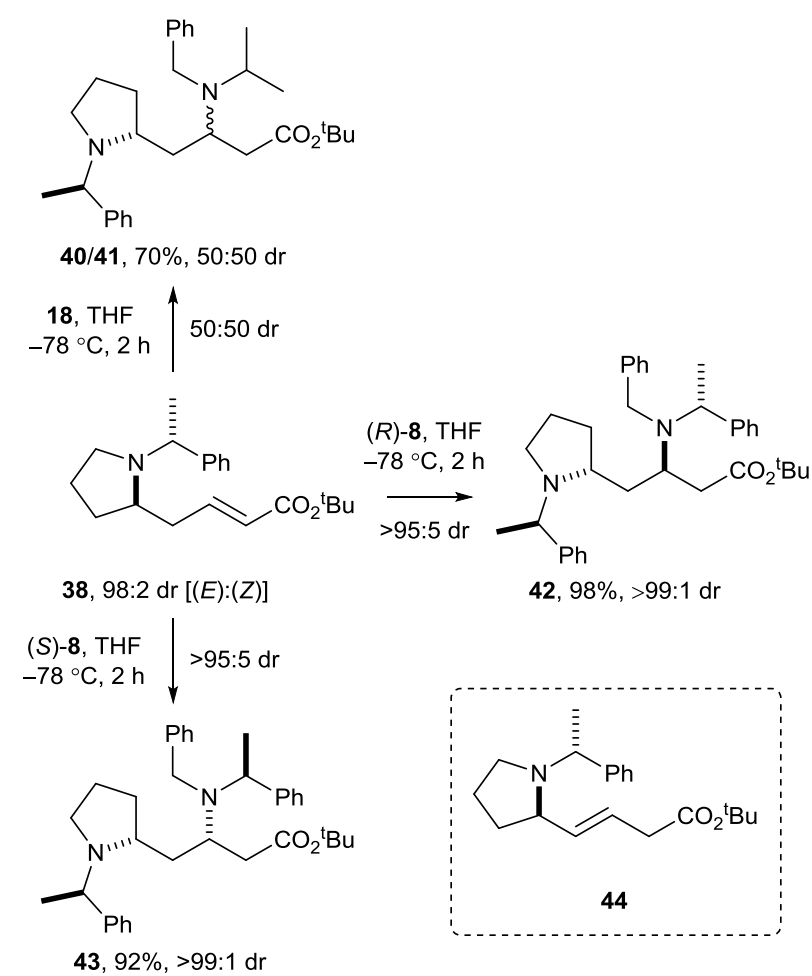

42, $98 \%,>99: 1 \mathrm{dr}$

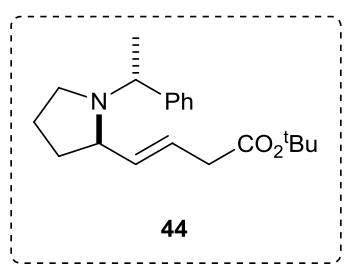

Reduction of 42 with DIBAL-H followed by Wittig reaction of the resultant aldehyde with the requisite ylid reagent $\mathrm{Ph}_{3} \mathrm{P}=\mathrm{CHR}$ gave either $45(\mathrm{R}=\mathrm{H})$ or $46(\mathrm{R}=\mathrm{Et})$; after chromatographic purification of the crude reaction mixtures, $\mathbf{4 5}$ was isolated in 76\% yield and >99:1 dr, and $\mathbf{4 6}$ was isolated in $75 \%$ yield and >95:5 $\mathrm{dr}[(Z):(E)]{ }^{20}$ Tandem hydrogenation/hydrogenolysis of $\mathbf{4 5}$ and $\mathbf{4 6}$ gave the corresponding diamines $\mathbf{4 7}$ and $\mathbf{4 8}$, which were immediately reacted with 4-bromobutanal ${ }^{6,9 \mathrm{~d}}$ to give (+)-tetraponerine 2 (49) and (+)-tetraponerine 6 (50) in 37 and 54\% yield, respectively, as single diastereoisomers (>99:1 dr) in each case (Scheme 7). For (+)-tetraponerine 2 (49), the specific rotation was consistent with literature values $\left\{[\alpha]_{\mathrm{D}}^{25}+44.2\left(c 1.0\right.\right.$ in $\left.\mathrm{CHCl}_{3}\right) ;$ lit. $^{5}[\alpha]_{\mathrm{D}}^{20}+36\left(\right.$ c 1.79 in $\left.\mathrm{CHCl}_{3}\right)$; lit. ${ }^{6}[\alpha]_{\mathrm{D}}^{20}+47\left(c 0.232\right.$ in $\left.\left.\mathrm{CHCl}_{3}\right)\right\}$; however, for $(+)$-tetraponerine $6(\mathbf{5 0})$ the specific rotations of our synthetic samples were consistently larger than literature values $\left\{[\alpha]_{\mathrm{D}}^{25}+66.3\left(c 1.0\right.\right.$ in $\left.\mathrm{CHCl}_{3}\right)$; lit. ${ }^{4}$ for a sample isolated from the natural source $[\alpha]_{\mathrm{D}}^{20}+35$ (c 0.15 in $\left.\mathrm{CHCl}_{3}\right)$; lit. ${ }^{6}[\alpha]_{\mathrm{D}}^{20}+40\left(c 0.75\right.$ in $\left.\left.\mathrm{CHCl}_{3}\right)\right\}$. Despite this discrepancy, ${ }^{22}$ the spectroscopic data for both samples of (+)-tetraponerine $2(\mathbf{4 9})$ and (+)-tetraponerine $6(\mathbf{5 0})$ were in good agreement with literature values, thereby confirming the assigned configurations of the synthetic precursors 42 and $45-48$. The 
asymmetric syntheses of (+)-tetraponerine $2(\mathbf{4 9})$ and (+)-tetraponerine $6(\mathbf{5 0})$ were therefore achieved in 9.6 and $13.8 \%$ overall yield, respectively, in nine steps from commercially available starting materials.

\section{SCHEME 7. Preparation of (+)-tetraponerine 2 and (+)-tetraponerine 6}

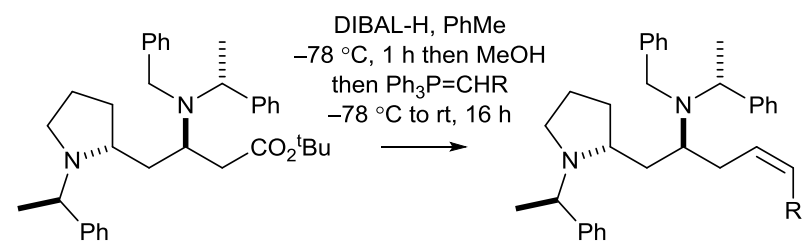

42, $>99: 1 \mathrm{dr}$

45, $\mathrm{R}=\mathrm{H}, 76 \%,>99: 1 \mathrm{dr}$

46, $\mathrm{R}=\mathrm{Et}, 75 \%,>95: 5 \mathrm{dr}[(Z):(E)]$

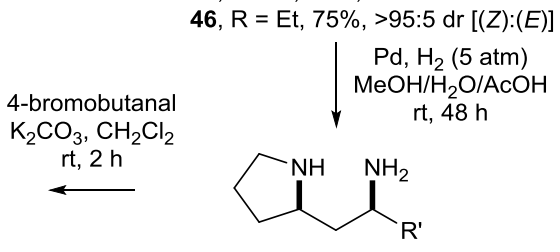

49, $\mathrm{R}^{\prime}=\mathrm{C}_{3} \mathrm{H}_{7},(+)$-tetraponerine 2

$37 \%$ (from 45), >99:1 dr

47, $\mathrm{R}^{\prime}=\mathrm{C}_{3} \mathrm{H}_{7}$, not isolated

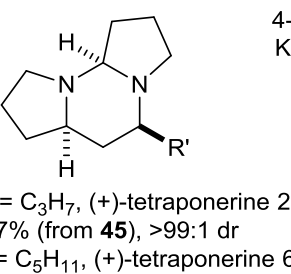

$\mathrm{Pd}, \mathrm{H}_{2}(5 \mathrm{~atm})$
$\mathrm{MeOH} / \mathrm{H}_{2} \mathrm{O} / \mathrm{AcOH}$

$54 \%$ (from 46), $>99: 1 \mathrm{dr}$

48, $\mathrm{R}^{\prime}=\mathrm{C}_{5} \mathrm{H}_{11}$, not isolated

Reduction of the diastereoisomeric substrate $\mathbf{4 3}$ with DIBAL-H followed by Wittig reaction of the resultant aldehyde with the requisite ylid reagent $\mathrm{Ph}_{3} \mathrm{P}=\mathrm{CHR}$ gave either $51(\mathrm{R}=\mathrm{H})$ or $52(\mathrm{R}=\mathrm{Et})$; after chromatographic purification of the crude reaction mixtures, $\mathbf{5 1}$ was isolated in $40 \%$ yield as a single diastereoisomer and $\mathbf{5 2}$ was isolated in $48 \%$ yield and $>95: 5 \mathrm{dr} \quad[(Z):(E)] .^{20}$ Tandem hydrogenation/hydrogenolysis of $\mathbf{5 1}$ and $\mathbf{5 2}$ gave the corresponding diamines $\mathbf{5 3}$ and 54, which were immediately reacted with 4-bromobutanal ${ }^{6,9 d}$ to give (+)-tetraponerine $1(\mathbf{5 5})$ and (+)-tetraponerine $5(\mathbf{5 6})$ in 15 and 14\% yield, respectively, as single diastereoisomers (>99:1 dr) in each case (Scheme 8). The spectroscopic data and specific rotations for these samples of (+)-tetraponerine $1(\mathbf{5 5})$ and (+)-tetraponerine $5(\mathbf{5 6})$ were in good agreement with literature values f for (+)-tetraponerine $1(\mathbf{5 5}):[\alpha]_{\mathrm{D}}^{25}+14.4(c 0.13$ in $\left.\mathrm{CHCl}_{3}\right)$; lit. ${ }^{5}[\alpha]_{\mathrm{D}}^{20}+11\left(\right.$ c 0.14 in $\left.\mathrm{CHCl}_{3}\right)$; lit. ${ }^{6}[\alpha]_{\mathrm{D}}^{20}+14$ ( 0.498 in $\left.\mathrm{CHCl}_{3}\right)$; for (+)-tetraponerine 5 (56): $[\alpha]_{\mathrm{D}}^{25}+12.4\left(c 0.13\right.$ in $\left.\mathrm{CHCl}_{3}\right)$; lit. ${ }^{4}$ for a sample isolated from the natural source $[\alpha]_{\mathrm{D}}^{20}+10\left(c 0.2\right.$ in $\left.\mathrm{CHCl}_{3}\right)$; lit. ${ }^{5}[\alpha]_{\mathrm{D}}^{20}+10(c) 0.24$ in $\left.\mathrm{CHCl}_{3}\right)$; lit. $^{6}[\alpha]_{\mathrm{D}}^{20}+14(c) 1.6$ in $\left.\mathrm{CHCl}_{3}\right)$ ), thereby confirming the assigned configurations of the synthetic precursors 43 and 51-54. The asymmetric syntheses of (+)-tetraponerine 1 (55) and (+)-tetraponerine 5 (56) were therefore achieved in 1.9 and 2.2\% overall yield, respectively, in nine steps from commercially available starting materials. 
55, $\mathrm{R}^{\prime}=\mathrm{C}_{3} \mathrm{H}_{7},(+)$-tetraponerine 1 $15 \%$ (from 51), >99:1 dr 56, $\mathrm{R}^{\prime}=\mathrm{C}_{5} \mathrm{H}_{11},(+)$-tetraponerine 5 $14 \%$ (from 52), >99:1 dr

SCHEME 8. Preparation of (+)-tetraponerine 1 and (+)-tetraponerine 5

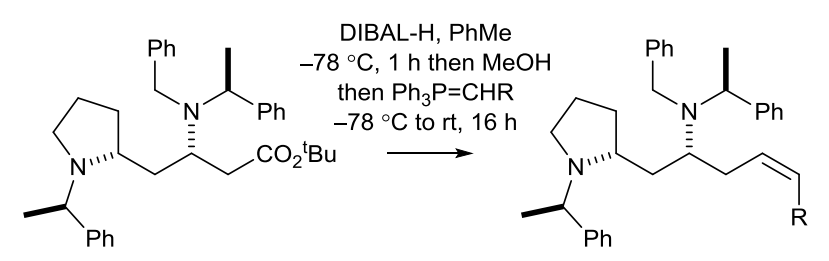

43, >99:1 dr

51, $\mathrm{R}=\mathrm{H}, 40 \%,>99: 1 \mathrm{dr}$ 52, $\mathrm{R}=\mathrm{Et}, 48 \%,>95: 5 \mathrm{dr}[(Z):(E)]$

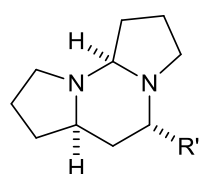

4-bromobutanal $\mathrm{K}_{2} \mathrm{CO}_{3}, \mathrm{CH}_{2} \mathrm{Cl}_{2}$ $\mathrm{rt}, 2 \mathrm{~h}$ $\mathrm{Pd}, \mathrm{H}_{2}(5 \mathrm{~atm})$ $\mathrm{MeOH} / \mathrm{H}_{2} \mathrm{O} / \mathrm{AcOH}$

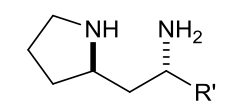

53, $\mathrm{R}^{\prime}=\mathrm{C}_{3} \mathrm{H}_{7}$, not isolated 54, $\mathrm{R}^{\prime}=\mathrm{C}_{5} \mathrm{H}_{11}$, not isolated

\section{Conclusion}

In conclusion, the conjugate addition of either lithium $(R)-N$-allyl- $N$-( $\alpha$-methylbenzyl)amide or lithium $(R)-N$-(but-3-en-1-yl)- $N$-( $\alpha$-methylbenzyl)amide to tert-butyl sorbate was followed by ring-closing metathesis of the resultant $N$-alkenyl $\beta$-amino esters and homologation. Subsequent conjugate addition of the requisite antipode of lithium $N$-benzyl- $N$-( $\alpha$-methylbenzyl)amide to the resultant $\alpha, \beta$-unsaturated esters gave a range of diamines for elaboration to all eight tetraponerine alkaloids (T1-T8) via a sequence involving reduction of the ester moiety to the corresponding aldehyde, olefination, tandem hydrogenation/hydrogenolysis, and reaction with 4-bromobutanal to give the tricyclic skeleton. In each case, the target alkaloids were isolated enantiomerically pure as single diastereoisomers in nine steps or fewer from commercially available sorbic acid.

\section{Experimental Section}

General experimental details. All reactions involving organometallic or other moisture-sensitive reagents were carried out under a nitrogen atmosphere using standard vacuum line techniques and glassware that was flame dried and cooled under nitrogen before use. Solvents were dried according to the procedure outlined by Grubbs and co-workers. ${ }^{23} \mathrm{BuLi}$ was purchased as a solution in hexanes and titrated against diphenylacetic acid before use. All other reagents were used as supplied without prior purification. Organic layers were dried over $\mathrm{Na}_{2} \mathrm{SO}_{4}$. Thin layer chromatography was performed on aluminium plates coated with $60 \mathrm{~F}_{254}$ silica. Plates were visualised using UV light $(254 \mathrm{~nm}), 1 \%$ aq $\mathrm{KMnO}_{4}$ or Dragendorff's reagent. Flash column chromatography was performed on Kieselgel 60 silica. Melting points are uncorrected. Specific rotations are reported in $10^{-1} \mathrm{deg}^{2} \mathrm{~cm}^{-1}$ and concentrations in $\mathrm{g} / 100 \mathrm{~mL}$. IR spectra were recorded 
using an ATR module. Selected characteristic peaks are reported in $\mathrm{cm}^{-1}$. NMR spectra were recorded in the deuterated solvent stated. Spectra were recorded at $\mathrm{rt}$ unless otherwise stated. The field was locked by external referencing to the relevant deuteron resonance. ${ }^{1} \mathrm{H}-{ }^{1} \mathrm{H} \mathrm{COSY},{ }^{1} \mathrm{H}-{ }^{13} \mathrm{C} \mathrm{HMQC}$, and ${ }^{1} \mathrm{H}-{ }^{13} \mathrm{C}$ HMBC analyses were used to establish atom connectivity. Accurate mass measurements were run on a TOF spectrometer internally calibrated with polyalanine.

tert-Butyl $(\boldsymbol{E}, \boldsymbol{E})$-hexa-2,4-dienoate [tert-butyl sorbate] 3. Condensed isobutylene $(180 \mathrm{~mL})$ at -78 ${ }^{\circ} \mathrm{C}$ was added to a stirred solution of sorbic acid $(30.0 \mathrm{~g}, 268 \mathrm{mmol})$ and conc aq $\mathrm{H}_{2} \mathrm{SO}_{4}(3.00 \mathrm{~mL})$ in $\mathrm{CH}_{2} \mathrm{Cl}_{2}(600 \mathrm{~mL})$ at $0{ }^{\circ} \mathrm{C}$, and the resultant mixture was allowed to warm to $\mathrm{rt}$ and stirred at $\mathrm{rt}$ for $48 \mathrm{~h}$. The reaction mixture was washed with satd aq $\mathrm{NaHCO}_{3}(5 \times 300 \mathrm{~mL})$ and the combined aqueous washings were extracted with $\mathrm{CH}_{2} \mathrm{Cl}_{2}(2 \times 300 \mathrm{~mL})$. The combined organic extracts were washed with brine $(500 \mathrm{~mL})$, then dried and concentrated in vacuo to give 3 as a pale yellow oil $(41.1 \mathrm{~g}, 91 \%,>99: 1 \mathrm{dr}) ;{ }^{13} \delta_{\mathrm{H}}(400 \mathrm{MHz}$, $\left.\mathrm{CDCl}_{3}\right) 1.48(9 \mathrm{H}, \mathrm{s}, \mathrm{CMe} 3), 1.84\left(3 \mathrm{H}, \mathrm{d}, J 5.9, \mathrm{C}(6) H_{3}\right), 5.70(1 \mathrm{H}, \mathrm{d}, J 15.4, \mathrm{C}(2) H), 6.04-6.21(2 \mathrm{H}, \mathrm{m}$, $\mathrm{C}(4) H, \mathrm{C}(5) H), 7.15(1 \mathrm{H}, \mathrm{dd}, J 15.4,10.0, \mathrm{C}(3) H)$.

tert-Butyl (3S, $\alpha R, E)-3-[N$-(but-3'-en-1'-yl)- $N$-( $\alpha$-methylbenzyl)amino]hex-4-enoate 13. BuLi (2.3 $\mathrm{M}$ in hexanes, $2.00 \mathrm{~mL}, 4.61 \mathrm{mmol})$ was added dropwise to a stirred solution of $(R)-N$-(but-3-en-1-yl)- $N-(\alpha-$ methlbenzyl)amine (833 mg, $4.76 \mathrm{mmol}$, >99:1 er) in $\mathrm{THF}(5 \mathrm{~mL})$ at $-78{ }^{\circ} \mathrm{C}$ and the resultant mixture was stirred at $-78{ }^{\circ} \mathrm{C}$ for $30 \mathrm{~min}$. A solution of $3(500 \mathrm{mg}, 2.97 \mathrm{mmol}$, >99:1 dr) in $\mathrm{THF}(2 \mathrm{~mL})$ at $-78{ }^{\circ} \mathrm{C}$ was added via cannula and the resultant mixture was stirred at $-78{ }^{\circ} \mathrm{C}$ for $2 \mathrm{~h}$. Satd aq $\mathrm{NH}_{4} \mathrm{Cl}(2 \mathrm{~mL})$ was added and the reaction mixture was allowed to warm to $\mathrm{rt}$, then concentrated in vacuo. The residue was partitioned between $\mathrm{CH}_{2} \mathrm{Cl}_{2}(10 \mathrm{~mL})$ and $10 \%$ citric acid $(10 \mathrm{~mL})$, and the organic layer was washed with satd aq $\mathrm{NaHCO}_{3}(10 \mathrm{~mL})$ and brine $(10 \mathrm{~mL})$, then dried and concentrated in vacuo to give $\mathbf{1 3}$ in $>95: 5 \mathrm{dr}$. Purification via flash column chromatography (eluent $30-40{ }^{\circ} \mathrm{C}$ petrol/Et $2 \mathrm{O}, 20: 1$ ) gave $\mathbf{1 3}$ as a pale yellow oil $(708 \mathrm{mg}, 69 \%,>99: 1 \mathrm{dr}) ;^{13}[\alpha]_{\mathrm{D}}^{25}-15.4$ (c 2.0 in $\left.\mathrm{CHCl}_{3}\right) ;\left\{\right.$ lit. $^{14}[\alpha]_{\mathrm{D}}^{20}-14.6$ (c 1.0 in $\left.\left.\mathrm{CHCl}_{3}\right)\right\} ; \delta_{\mathrm{H}}(400$ $\left.\mathrm{MHz}, \mathrm{CDCl}_{3}\right) 1.36(3 \mathrm{H}, \mathrm{d}, J$ 6.7, C( $\left.\alpha) M e\right), 1.40(9 \mathrm{H}, \mathrm{s}, \mathrm{CMe} 3), 1.69\left(3 \mathrm{H}, \mathrm{d}, J\right.$ 5.0, C(6)H $\left.H_{3}\right), 1.96-2.08(2 \mathrm{H}$, m, C(2') $\left.H_{2}\right), 2.28\left(1 \mathrm{H}, \mathrm{dd}, J 14.1,8.2, \mathrm{C}(2) H_{\mathrm{A}}\right), 2.40\left(1 \mathrm{H}, \mathrm{dd}, J 14.1,6.6, \mathrm{C}(2) H_{\mathrm{B}}\right), 2.44-2.58(2 \mathrm{H}, \mathrm{m}$, $\left.\mathrm{C}\left(1^{\prime}\right) H_{2}\right), 3.75-3.81(1 \mathrm{H}, \mathrm{m}, \mathrm{C}(3) H), 3.93(1 \mathrm{H}, \mathrm{q}, J 6.7, \mathrm{C}(\alpha) H), 4.86-4.94\left(2 \mathrm{H}, \mathrm{m}, \mathrm{C}\left(4^{\prime}\right) H_{2}\right), 5.45-5.58(2 \mathrm{H}$, m, C(4)H, C(5)H), $5.64\left(1 \mathrm{H}, \mathrm{ddt}, J 17.0,10.3,6.8, \mathrm{C}\left(3^{\prime}\right) H\right), 7.17-7.23(1 \mathrm{H}, \mathrm{m}, P h), 7.25-7.30(2 \mathrm{H}, \mathrm{m}, P h)$, 7.32-7.37 (2H, m, Ph).

tert-Butyl $\quad\left(2^{\prime} S, \alpha R\right)-2-\left[N\left(1^{\prime}\right)-(\alpha-m e t h y l b e n z y l)-1^{\prime}, 2^{\prime}, 5^{\prime}, 6^{\prime}\right.$-tetrahydropyridin-2'-yl]acetate 14. Method A (from 13): Grubbs I catalyst (301 mg, $0.366 \mathrm{mmol}$ ) was added to a stirred, degassed solution of 13 (3.14 g, $9.14 \mathrm{mmol},>99: 1 \mathrm{dr})$ in anhydrous $\mathrm{CH}_{2} \mathrm{Cl}_{2}(\mathrm{EtOH}$ stabilised, $300 \mathrm{~mL})$ at rt. The resultant mixture 
was stirred at $\mathrm{rt}$ for $16 \mathrm{~h}$, then concentrated in vacuo. Purification via flash column chromatography (eluent 30-40 ${ }^{\circ} \mathrm{C}$ petrol/ $\left.\mathrm{Et}_{2} \mathrm{O} / \mathrm{NH}_{4} \mathrm{OH}, 100: 10: 1\right)$ gave 14 as a pale yellow oil (2.30 g, 83\% from 13, >99:1 dr); ${ }^{13}$ $[\alpha]_{\mathrm{D}}^{25}+41.5\left(c 1.0\right.$ in $\left.\mathrm{CHCl}_{3}\right) ;\left\{\right.$ lit. $^{13}[\alpha]_{\mathrm{D}}^{25}+41.8\left(c 1.5\right.$ in $\left.\left.\mathrm{CHCl}_{3}\right)\right\} ; \delta_{\mathrm{H}}\left(400 \mathrm{MHz}, \mathrm{CDCl}_{3}\right) 1.38(3 \mathrm{H}, \mathrm{d}, J 6.6$, $\mathrm{C}(\alpha) M e), 1.48\left(9 \mathrm{H}, \mathrm{s}, \mathrm{C} M e_{3}\right), 1.66-1.75\left(1 \mathrm{H}, \mathrm{m}, \mathrm{C}\left(5^{\prime}\right) H_{\mathrm{A}}\right), 2.04-2.16\left(1 \mathrm{H}, \mathrm{m}, \mathrm{C}\left(5^{\prime}\right) H_{\mathrm{B}}\right), 2.37(1 \mathrm{H}, \mathrm{dd}, J 14.2$, 6.8, C(2) $\left.H_{\mathrm{A}}\right), 2.43-2.50\left(1 \mathrm{H}, \mathrm{m}, \mathrm{C}\left(6^{\prime}\right) H_{\mathrm{A}}\right), 2.59\left(1 \mathrm{H}, \mathrm{dd}, J 14.2,7.3, \mathrm{C}(2) H_{\mathrm{B}}\right), 2.84(1 \mathrm{H}, \mathrm{ddd}, J 13.9,9.5,4.7$, $\left.\mathrm{C}\left(6^{\prime}\right) H_{\mathrm{B}}\right), 3.70-3.77\left(1 \mathrm{H}, \mathrm{m}, \mathrm{C}\left(2^{\prime}\right) H\right), 3.89(1 \mathrm{H}, \mathrm{q}, J 6.6, \mathrm{C}(\alpha) H), 5.62-5.67\left(1 \mathrm{H}, \mathrm{m}, \mathrm{C}\left(3^{\prime}\right) H\right), 5.78-5.85(1 \mathrm{H}$, $\left.\mathrm{m}, \mathrm{C}\left(4^{\prime}\right) H\right), 7.20-7.34(5 \mathrm{H}, \mathrm{m}, P h)$.

Method B (from 3) - Step 1: BuLi (2.3 M in hexanes, $20.0 \mathrm{~mL}, 46.1 \mathrm{mmol})$ was added dropwise to a stirred solution of $(R)-N$-(but-3-en-1-yl)- $N$-( $\alpha$-methylbenzyl)amine ( $8.33 \mathrm{~g}, 47.6 \mathrm{mmol},>99: 1 \mathrm{er})$ in THF (70 mL) at $-78{ }^{\circ} \mathrm{C}$ and the resultant mixture was stirred at $-78{ }^{\circ} \mathrm{C}$ for $30 \mathrm{~min}$. A solution of $\mathbf{3}(5.00 \mathrm{~g}, 29.7 \mathrm{mmol}$, $>99: 1 \mathrm{dr})$ in THF $(10 \mathrm{~mL})$ at $-78{ }^{\circ} \mathrm{C}$ was then added and the resultant mixture was stirred at $-78{ }^{\circ} \mathrm{C}$ for $2 \mathrm{~h}$. Satd aq $\mathrm{NH}_{4} \mathrm{Cl}(20 \mathrm{~mL})$ was added and the reaction mixture was allowed to warm to $\mathrm{rt}$, then concentrated in vacuo. The residue was partitioned between $\mathrm{CH}_{2} \mathrm{Cl}_{2}(50 \mathrm{~mL})$ and $10 \%$ citric acid $(50 \mathrm{~mL})$, and the organic layer was washed with satd aq $\mathrm{NaHCO}_{3}(50 \mathrm{~mL})$ and brine $(50 \mathrm{~mL})$, then dried and concentrated in vacuo to give 13 in >95:5 dr.

Method B (from 3) - Step 2: Grubbs I catalyst (854 mg, $1.04 \mathrm{mmol}$ ) was added to a stirred, degassed solution of the residue of $\mathbf{1 3}$ from the previous step in anhydrous $\mathrm{CH}_{2} \mathrm{Cl}_{2}(\mathrm{EtOH}$ stabilised, $900 \mathrm{~mL})$ at $\mathrm{rt}$. The resultant solution was stirred at $\mathrm{rt}$ for $16 \mathrm{~h}$, then concentrated in vacuo. Purification via flash column chromatography (eluent $30-40{ }^{\circ} \mathrm{C}$ petrol/ $\left./ \mathrm{Et}_{2} \mathrm{O} / \mathrm{NH}_{4} \mathrm{OH}, 100: 10: 1\right)$ gave 14 as a pale yellow oil $(6.55 \mathrm{~g}, 73 \%$ from 3, >99.1 dr); $[\alpha]_{\mathrm{D}}^{25}+44.8\left(c 0.8\right.$ in $\left.\mathrm{CHCl}_{3}\right)$.

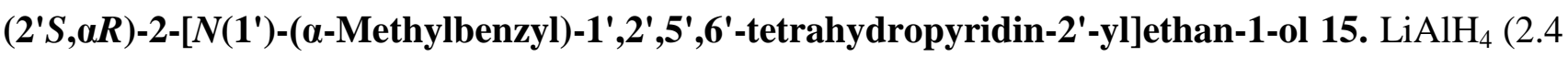
$\mathrm{M}$ in THF, $3.18 \mathrm{~mL}, 7.63 \mathrm{mmol})$ was added to a stirred solution of $\mathbf{1 4}(2.30 \mathrm{~g}, 7.63 \mathrm{mmol},>99: 1 \mathrm{dr})$ in THF $(70 \mathrm{~mL})$ at $0{ }^{\circ} \mathrm{C}$. The resultant mixture was allowed to warm to $\mathrm{rt}$ and stirred at $\mathrm{rt}$ for $16 \mathrm{~h} .2 .0 \mathrm{M}$ aq $\mathrm{NaOH}$ $(7 \mathrm{~mL})$ was then added and the resultant mixture was stirred at $\mathrm{rt}$ for $3 \mathrm{~h}$. The reaction mixture was filtered through Celite ${ }^{\circledR}$ (eluent $\mathrm{CH}_{2} \mathrm{Cl}_{2}$ ), then concentrated in vacuo to give 15 as a pale yellow oil (1.66 g, 94\%, >99:1 dr); $[\alpha]_{\mathrm{D}}^{25}-3.9$ (c 1.0 in $\left.\mathrm{CHCl}_{3}\right) ; v_{\max }(\mathrm{ATR}) 3393(\mathrm{O}-\mathrm{H}), 3026,2970,2839,(\mathrm{C}-\mathrm{H}), 1454(\mathrm{C}=\mathrm{C}) ; \delta_{\mathrm{H}}$ $\left(400 \mathrm{MHz}, \mathrm{CDCl}_{3}\right) 1.49(3 \mathrm{H}, \mathrm{d}, J$ 6.6, C( $\left.\alpha) M e\right), 1.73-1.88\left(2 \mathrm{H}, \mathrm{m}, \mathrm{C}(2) H_{\mathrm{A}}, \mathrm{C}\left(5^{\prime}\right) H_{\mathrm{A}}\right), 1.88-1.97(1 \mathrm{H}, \mathrm{m}$, $\left.\mathrm{C}(2) H_{\mathrm{B}}\right), 2.04-2.14\left(1 \mathrm{H}, \mathrm{m}, \mathrm{C}\left(5^{\prime}\right) H_{\mathrm{B}}\right), 2.34\left(1 \mathrm{H}\right.$, app dt, $\left.J 13.4,4.5, \mathrm{C}\left(6^{\prime}\right) H_{\mathrm{A}}\right), 3.02(1 \mathrm{H}, \mathrm{ddd}, J 13.4,8.9,4.7$, $\left.\mathrm{C}\left(6^{\prime}\right) H_{\mathrm{B}}\right), 3.59-3.66\left(1 \mathrm{H}, \mathrm{m}, \mathrm{C}\left(2^{\prime}\right) H\right), 3.85-3.96\left(2 \mathrm{H}, \mathrm{m}, \mathrm{C}(1) H_{2}\right), 4.05(1 \mathrm{H}, \mathrm{q}, J 6.6, \mathrm{C}(\alpha) H), 5.50-5.56(1 \mathrm{H}$, m, C(3')H), 5.84-5.97 (1H, m, C(4')H), $6.36\left(1 \mathrm{H}\right.$, br s, OH), 7.24-7.39 (5H, m, Ph); $\delta_{\mathrm{C}}\left(100 \mathrm{MHz}, \mathrm{CDCl}_{3}\right)$ 21.3, $21.4\left(C\left(5^{\prime}\right), \mathrm{C}(\alpha) M e\right), 32.9(C(2)), 40.0\left(C\left(6^{\prime}\right)\right), 56.2\left(C\left(2^{\prime}\right)\right), 57.6(C(\alpha)), 63.0(C(1)), 126.4\left(C\left(4^{\prime}\right)\right)$, 
$127.4(p-P h), 127.8,128.5(o, m-P h), 128.5\left(C\left(3^{\prime}\right)\right), 142.6(i-P h) ; m / z\left(\mathrm{ESI}^{+}\right) 232\left([\mathrm{M}+\mathrm{H}]^{+}, 100 \%\right)$; HRMS $\left(\mathrm{ESI}^{+}\right) m / z:[\mathrm{M}+\mathrm{H}]^{+}$Calcd for $\mathrm{C}_{15} \mathrm{H}_{22} \mathrm{NO}^{+} 232.1696$; Found 232.1696.

tert-Butyl $(2 ' S, \alpha R, E)-4-\left[N\left(1^{\prime}\right)-(\alpha-m e t h y l b e n z y l)-1 ', 2 ', 5^{\prime}, 6^{\prime}\right.$-tetrahydropyridin-2'-yl]but-2-enoate 16 and tert-Butyl $\left(2^{\prime} S, \alpha R, Z\right)-4-\left[N\left(1^{\prime}\right)-(\alpha-m e t h y l b e n z y l)-1^{\prime}, 2^{\prime}, 5^{\prime}, 6^{\prime}\right.$-tetrahydropyridin-2'-yl]but-2-enoate 17. Method A (from 15): DMSO $(0.61 \mathrm{~mL}, 8.65 \mathrm{mmol})$ was added to a stirred solution of $(\mathrm{COCl})_{2}(0.37 \mathrm{~mL}$, $4.32 \mathrm{mmol})$ in $\mathrm{CH}_{2} \mathrm{Cl}_{2}(50 \mathrm{~mL})$ at $-78{ }^{\circ} \mathrm{C}$. After $20 \mathrm{~min}, 15(500 \mathrm{mg}, 2.16 \mathrm{mmol},>99: 1 \mathrm{dr})$ was added and the resultant mixture was stirred at $-78{ }^{\circ} \mathrm{C}$ for $40 \mathrm{~min}$, then $\mathrm{Et}_{3} \mathrm{~N}(1.81 \mathrm{~mL}, 13.0 \mathrm{mmol})$ was added. The reaction mixture was allowed to warm to $\mathrm{rt}, \mathrm{Ph}_{3} \mathrm{P}=\mathrm{CHCO}_{2}{ }^{\mathrm{t}} \mathrm{Bu}(814 \mathrm{mg}, 2.16 \mathrm{mmol})$ was added, and the resultant mixture was stirred at $\mathrm{rt}$ for $16 \mathrm{~h}$. Satd aq $\mathrm{K}_{2} \mathrm{CO}_{3}(300 \mathrm{~mL})$ was then added and the reaction mixture was extracted with $\mathrm{CH}_{2} \mathrm{Cl}_{2}(3 \times 300 \mathrm{~mL})$. The combined organic extracts were washed with brine $(500 \mathrm{~mL})$, then dried and concentrated in vacuo to give a 93:7 mixture of $\mathbf{1 6}$ and 17, respectively. Purification via flash column chromatography (eluent $30-40{ }^{\circ} \mathrm{C}$ petrol/Et $\left.2 \mathrm{O} / \mathrm{NH}_{4} \mathrm{OH}, 100: 10: 1\right)$ gave 17 as a pale yellow oil (19 mg, 3\%, >99:1 dr); $[\alpha]_{\mathrm{D}}^{25}+57.4\left(\right.$ c 1.0 in $\left.\mathrm{CHCl}_{3}\right) ; v_{\max }(\mathrm{ATR})$ 2975, $2931(\mathrm{C}-\mathrm{H}), 1712(\mathrm{C}=\mathrm{O}), 1639(\mathrm{C}=\mathrm{C})$; $\delta_{\mathrm{H}}\left(400 \mathrm{MHz}, \mathrm{CDCl}_{3}\right) 1.34(3 \mathrm{H}, \mathrm{d}, J$ 6.8, C( $\left.\alpha) M e\right), 1.47(9 \mathrm{H}, \mathrm{s}, \mathrm{CMe} 3), 1.84-1.94\left(1 \mathrm{H}, \mathrm{m}, \mathrm{C}\left(5^{\prime}\right) H_{\mathrm{A}}\right), 1.96-$ $2.05\left(1 \mathrm{H}, \mathrm{m}, \mathrm{C}\left(5^{\prime}\right) H_{\mathrm{B}}\right), 2.23\left(1 \mathrm{H}, \mathrm{ddd}, J 12.1,7.2,5.7, \mathrm{C}\left(6^{\prime}\right) H_{\mathrm{A}}\right), 2.65-2.73\left(1 \mathrm{H}, \mathrm{m}, \mathrm{C}(4) H_{\mathrm{A}}\right), 2.92(1 \mathrm{H}$, app $\left.\mathrm{dt}, J 12.1,5.6, \mathrm{C}\left(6^{\prime}\right) H_{\mathrm{B}}\right), 3.16-3.26\left(2 \mathrm{H}, \mathrm{m}, \mathrm{C}(4) H_{\mathrm{B}}, \mathrm{C}\left(2^{\prime}\right) H\right), 4.00(1 \mathrm{H}, \mathrm{q}, J 6.8, \mathrm{C}(\alpha) H), 5.46-5.51(1 \mathrm{H}, \mathrm{m}$, $\left.\mathrm{C}\left(3^{\prime}\right) H\right), 5.73(1 \mathrm{H}, \mathrm{d}, J 11.4, \mathrm{C}(2) H), 5.73-5.78\left(1 \mathrm{H}, \mathrm{m}, \mathrm{C}\left(4^{\prime}\right) H\right), 6.30(1 \mathrm{H}, \mathrm{app} \mathrm{dt}, J 11.4,7.5, \mathrm{C}(3) H), 7.17-$ $7.34(5 \mathrm{H}, \mathrm{m}, P h) ; \delta_{\mathrm{C}}\left(100 \mathrm{MHz}, \mathrm{CDCl}_{3}\right) 20.9(\mathrm{C}(\alpha) M e), 24.3\left(C\left(5^{\prime}\right)\right), 28.4\left(\mathrm{CMe}_{3}\right), 32.1(C(4)), 40.8\left(C\left(6^{\prime}\right)\right)$, $54.9\left(C\left(2^{\prime}\right)\right), 57.1(C(\alpha)), 80.1\left(C \mathrm{Me}_{3}\right), 122.0(C(2)), 126.5\left(C\left(4^{\prime}\right)\right), 126.9(p-P h), 128.1,128.1(o, m-P h)$, $130.1\left(C\left(3^{\prime}\right)\right), 142.6(i-P h), 146.7(C(3)), 166.4(C(1)) ; m / z\left(\mathrm{ESI}^{+}\right) 328\left([\mathrm{M}+\mathrm{H}]^{+}, 100 \%\right) ; \mathrm{HRMS}\left(\mathrm{ESI}^{+}\right) \mathrm{m} / z$ : $[\mathrm{M}+\mathrm{H}]^{+}$Calcd for $\mathrm{C}_{21} \mathrm{H}_{30} \mathrm{NO}_{2}{ }^{+}$328.2271; Found 328.2273. Further elution gave 16 as a yellow oil (322 mg, 46\%, >99:1 dr); $[\alpha]_{\mathrm{D}}^{25}+65.9\left(\right.$ c 1.0 in $\left.\mathrm{CHCl}_{3}\right) ; v_{\max }(\mathrm{ATR}) 2979,2932(\mathrm{C}-\mathrm{H}), 1713(\mathrm{C}=\mathrm{O}), 1651(\mathrm{C}=\mathrm{C}) ; \delta_{\mathrm{H}}$ $\left(400 \mathrm{MHz}, \mathrm{CDCl}_{3}\right) 1.38(3 \mathrm{H}, \mathrm{d}, J$ 6.7, C( $\left.\left.\alpha) M e\right), 1.49(9 \mathrm{H}, \mathrm{s}, \mathrm{CMe})_{3}\right), 1.82-1.93\left(1 \mathrm{H}, \mathrm{m}, \mathrm{C}\left(5^{\prime}\right) H_{\mathrm{A}}\right), 2.03-2.15$ $\left(1 \mathrm{H}, \mathrm{m}, \mathrm{C}\left(5^{\prime}\right) H_{\mathrm{B}}\right), 2.36-2.55\left(3 \mathrm{H}, \mathrm{m}, \mathrm{C}(4) H_{2}, \mathrm{C}\left(6^{\prime}\right) H_{\mathrm{A}}\right), 2.88\left(1 \mathrm{H}, \mathrm{ddd}, J 12.7,7.8,4.8, \mathrm{C}\left(6^{\prime}\right) H_{\mathrm{B}}\right), 3.23-3.31$ $\left(1 \mathrm{H}, \mathrm{m}, \mathrm{C}\left(2^{\prime}\right) H\right), 3.95(1 \mathrm{H}, \mathrm{q}, J$ 6.7, C( $(\alpha) H), 5.53-5.58\left(1 \mathrm{H}, \mathrm{m}, \mathrm{C}\left(3^{\prime}\right) H\right), 5.77(1 \mathrm{H}, \mathrm{d}, J 15.5, \mathrm{C}(2) H), 5.77-$ $5.83\left(1 \mathrm{H}, \mathrm{m}, \mathrm{C}\left(4^{\prime}\right) H\right), 6.90(1 \mathrm{H}, \mathrm{ddd}, J 15.5,8.0,6.7, \mathrm{C}(3) H), 7.21-7.35(5 \mathrm{H}, \mathrm{m}, P h) ; \delta_{\mathrm{C}}\left(100 \mathrm{MHz}, \mathrm{CDCl}_{3}\right)$ $21.4(\mathrm{C}(\alpha) M e), 23.9\left(C\left(5^{\prime}\right)\right), 28.3\left(\mathrm{CMe}_{3}\right), 35.9(C(4)), 40.7\left(C\left(6^{\prime}\right)\right), 54.6\left(C\left(2^{\prime}\right)\right), 57.9(C(\alpha)), 80.1\left(C \mathrm{Me}_{3}\right)$, $124.3(C(2)), 126.5\left(C\left(4^{\prime}\right)\right), 127.0(p-P h), 127.9,128.3(o, m-P h), 129.5\left(C\left(3^{\prime}\right)\right), 143.4(i-P h), 145.9(C(3))$, $166.1(C(1)) ; m / z\left(\mathrm{ESI}^{+}\right) 328\left([\mathrm{M}+\mathrm{H}]^{+}, 100 \%\right) ; \operatorname{HRMS}\left(\mathrm{ESI}^{+}\right) \mathrm{m} / z:[\mathrm{M}+\mathrm{H}]^{+} \mathrm{Calcd}$ for $\mathrm{C}_{21} \mathrm{H}_{30} \mathrm{NO}_{2}{ }^{+}$ 328.2271; Found 328.2271. 
Method B (from 14): DIBAL-H (1.0 M in PhMe, $7.30 \mathrm{~mL}, 7.30 \mathrm{mmol}$ ) was added dropwise to a stirred solution of $14(2.00 \mathrm{~g}, 6.64 \mathrm{mmol},>99: 1 \mathrm{dr})$ in $\mathrm{PhMe}(20 \mathrm{~mL})$ at $-78{ }^{\circ} \mathrm{C}$ and the resultant mixture was stirred at $-78{ }^{\circ} \mathrm{C}$ for $1 \mathrm{~h}$. $\mathrm{MeOH}(1.34 \mathrm{~mL}, 33.2 \mathrm{mmol})$ and $\mathrm{Ph}_{3} \mathrm{P}=\mathrm{CHCO}_{2}{ }^{\mathrm{B}} \mathrm{Bu}(2.50 \mathrm{~g}, 6.64 \mathrm{mmol})$ were added sequentially, and the resultant mixture was allowed to warm to $\mathrm{rt}$ and stirred at $\mathrm{rt}$ for $16 \mathrm{~h}$. The reaction mixture was then concentrated in vacuo to give an 80:20 mixture of $\mathbf{1 6}$ and 17, respectively. Purification via flash column chromatography (eluent $30-40{ }^{\circ} \mathrm{C}$ petrol/ $\mathrm{Et}_{2} \mathrm{O} / \mathrm{NH}_{4} \mathrm{OH}, 100: 10: 1$ ) gave 17 as a pale yellow oil $(313 \mathrm{mg}, 14 \%,>99: 1 \mathrm{dr}) ;[\alpha]_{\mathrm{D}}^{25}+60.2\left(c 0.5\right.$ in $\left.\mathrm{CHCl}_{3}\right)$. Further elution gave 16 as a yellow oil $(1.57 \mathrm{~g}, 72 \%,>99: 1 \mathrm{dr}) ;[\alpha]_{\mathrm{D}}^{25}+63.4\left(c 1.0\right.$ in $\left.\mathrm{CHCl}_{3}\right)$.

tert-Butyl $\quad\left(3 R, 2^{\prime} S, \alpha R\right)-3-[N-$ benzyl- $N$-isopropylamino]-4-[N(1')-( $\alpha$-methylbenzyl)-1',2',5',6'tetrahydropyridin-2'-yl]butanoate and tert-butyl $\left(3 S, 2^{\prime} S, \alpha R\right)-3-[N$-benzyl- $N$-isopropylamino]-4-[N(1')(a-methylbenzyl)-1',2',5',6'-tetrahydropyridin-2'-yl]butanoate 19 and 20. BuLi (2.3 M in hexanes, 0.44 $\mathrm{mL}, 1.00 \mathrm{mmol}$ ) was added dropwise to a stirred solution of $N$-benzyl- $N$-isopropylamine (155 mg, 1.04 $\mathrm{mmol})$ in THF $(2 \mathrm{~mL})$ at $-78{ }^{\circ} \mathrm{C}$ and the resultant mixture was stirred at $-78{ }^{\circ} \mathrm{C}$ for $30 \mathrm{~min}$. A solution of 16 (212 mg, $0.647 \mathrm{mmol},>99: 1 \mathrm{dr})$ in $\mathrm{THF}(1 \mathrm{~mL})$ at $-78^{\circ} \mathrm{C}$ was added via cannula and the resultant mixture was stirred at $-78{ }^{\circ} \mathrm{C}$ for $2 \mathrm{~h}$. Satd aq $\mathrm{NH}_{4} \mathrm{Cl}(0.5 \mathrm{~mL})$ was added and the reaction mixture was allowed to warm to rt, then concentrated in vacuo. The residue was partitioned between $\mathrm{CH}_{2} \mathrm{Cl}_{2}(10 \mathrm{~mL})$ and $10 \%$ citric acid $(10 \mathrm{~mL})$, and the organic layer was washed with satd aq $\mathrm{NaHCO}_{3}(10 \mathrm{~mL})$ and brine $(10 \mathrm{~mL})$, then dried and concentrated in vacuo to give a 50:50 mixture of $\mathbf{1 9}$ and $\mathbf{2 0}$ as a colourless oil (296 mg, 96\%). Data for 19: $\delta_{\mathrm{H}}\left(400 \mathrm{MHz}, \mathrm{CDCl}_{3}\right) 1.05-1.11(6 \mathrm{H}, \mathrm{m}, \mathrm{NCHMe}), 1.35(3 \mathrm{H}, \mathrm{d}, J$ 6.5, $\mathrm{C}(\alpha) M e), 1.36-1.63(2 \mathrm{H}, \mathrm{m}$, $\left.\mathrm{C}(4) H_{\mathrm{A}}, \mathrm{C}\left(5^{\prime}\right) H_{\mathrm{A}}\right), 1.50\left(9 \mathrm{H}, \mathrm{s}, \mathrm{C} M e_{3}\right), 1.84-1.98\left(1 \mathrm{H}, \mathrm{m}, \mathrm{C}(4) H_{\mathrm{B}}\right), 2.04-2.17\left(1 \mathrm{H}, \mathrm{m}, \mathrm{C}\left(5^{\prime}\right) H_{\mathrm{B}}\right), 2.42-2.57$ $\left(3 \mathrm{H}, \mathrm{m}, \mathrm{C}(2) H_{2}, \mathrm{C}\left(6^{\prime}\right) H_{\mathrm{A}}\right), 2.72-2.84\left(1 \mathrm{H}, \mathrm{m}, \mathrm{C}\left(6^{\prime}\right) H_{\mathrm{B}}\right), 2.91-3.02(1 \mathrm{H}, \mathrm{m}, \mathrm{NCHMe}), 3.32-3.43(1 \mathrm{H}, \mathrm{m}$, $\left.\mathrm{C}\left(2^{\prime}\right) H\right), 3.52-3.61(1 \mathrm{H}, \mathrm{m}, \mathrm{C}(3) H), 3.65\left(1 \mathrm{H}, \mathrm{d}, J 14.5, \mathrm{NCH}_{\mathrm{A}} \mathrm{H}_{\mathrm{B}} \mathrm{Ph}\right), 3.73\left(1 \mathrm{H}, \mathrm{d}, J 14.5, \mathrm{NCH}_{\mathrm{A}} H_{\mathrm{B}} \mathrm{Ph}\right), 3.84$ $(1 \mathrm{H}, \mathrm{q}, J$ 6.5, C $(\alpha) H), 5.33-5.43\left(1 \mathrm{H}, \mathrm{m}, \mathrm{C}\left(3^{\prime}\right) H\right), 5.69-5.79\left(1 \mathrm{H}, \mathrm{m}, \mathrm{C}\left(4^{\prime}\right) H\right), 7.19-7.43(10 \mathrm{H}, \mathrm{m}, P h)$. Data for 20: $\delta_{\mathrm{H}}\left(400 \mathrm{MHz}, \mathrm{CDCl}_{3}\right) 1.05-1.11(6 \mathrm{H}, \mathrm{m}, \mathrm{NCHMe}), 1.35(3 \mathrm{H}, \mathrm{d}, J$ 6.5, $\mathrm{C}(\alpha) M e), 1.36-1.63(2 \mathrm{H}, \mathrm{m}$, $\left.\mathrm{C}(4) H_{\mathrm{A}}, \mathrm{C}\left(5^{\prime}\right) H_{\mathrm{A}}\right), 1.46\left(9 \mathrm{H}, \mathrm{s}, \mathrm{C} M e_{3}\right), 1.68\left(1 \mathrm{H}\right.$, app dt, $\left.J 14.2,5.2, \mathrm{C}(4) H_{\mathrm{B}}\right), 2.04-2.17\left(1 \mathrm{H}, \mathrm{m}, \mathrm{C}\left(5^{\prime}\right) H_{\mathrm{B}}\right)$, $2.29\left(1 \mathrm{H}, \mathrm{dd}, J 13.7,6.2, \mathrm{C}(2) H_{\mathrm{A}}\right), 2.40-2.57\left(2 \mathrm{H}, \mathrm{m}, \mathrm{C}(2) H_{\mathrm{B}}, \mathrm{C}\left(6^{\prime}\right) H_{\mathrm{A}}\right), 2.72-2.84\left(1 \mathrm{H}, \mathrm{m}, \mathrm{C}\left(6^{\prime}\right) H_{\mathrm{B}}\right), 2.91-$ $3.02(1 \mathrm{H}, \mathrm{m}, \mathrm{NCHMe}), 3.32-3.43\left(1 \mathrm{H}, \mathrm{m}, \mathrm{C}\left(2^{\prime}\right) H\right), 3.52-3.61(1 \mathrm{H}, \mathrm{m}, \mathrm{C}(3) H), 3.66\left(2 \mathrm{H}, \mathrm{s}, \mathrm{NCH} \mathrm{H}_{2} \mathrm{Ph}\right), 3.84$ $(1 \mathrm{H}, \mathrm{q}, J$ 6.5, C( $(\alpha) H), 5.49-5.55\left(1 \mathrm{H}, \mathrm{m}, \mathrm{C}\left(3^{\prime}\right) H\right), 5.69-5.79\left(1 \mathrm{H}, \mathrm{m}, \mathrm{C}\left(4^{\prime}\right) H\right), 7.19-7.43(10 \mathrm{H}, \mathrm{m}, P h)$. Data for mixture of 19 and 20: $v_{\max }(\mathrm{ATR}) 3024,2967,2931,2837(\mathrm{C}-\mathrm{H}), 1724(\mathrm{C}=\mathrm{O}), 1602(\mathrm{C}=\mathrm{C})$; $\delta_{\mathrm{C}}(100$ $\left.\mathrm{MHz}, \mathrm{CDCl}_{3}\right)$ 20.2, 20.4, 20.8, 20.9, 21.1, 21.8, 22.7, 23.0, 28.3, 28.3, 36.5, 37.4, 39.8, 39.9, 40.2, 40.4, $47.8,49.3,49.4,50.0,51.2,51.9,52.4,53.9,58.0,58.2,80.0,80.1,125.4,125.7,126.6,126.6,126.8,126.8$, 
$127.5,127.6,128.1,128.1,128.3,128.3,128.5,129.0,129.8,130.2,141.8,142.3,145.9,146.2,172.3$, 172.6; $m / z\left(\mathrm{ESI}^{+}\right) 477\left([\mathrm{M}+\mathrm{H}]^{+}, 100 \%\right)$; HRMS $\left(\mathrm{ESI}^{+}\right) \mathrm{m} / z:[\mathrm{M}+\mathrm{H}]^{+}$Calcd for $\mathrm{C}_{31} \mathrm{H}_{45} \mathrm{~N}_{2} \mathrm{O}_{2}{ }^{+} 477.3476$; Found 477.3471.

tert-Butyl $\quad\left(3 R, 2^{\prime} S, \alpha R, \alpha^{\prime} R\right)-3-\left[N\right.$-benzyl- $N$-( $\alpha$-methylbenzyl)amino]-4-[ $N\left(1^{\prime}\right)$-( $\alpha^{\prime}$-methylbenzyl)1',2',5',6'-tetrahydropyridin-2'-yl]butanoate 21. BuLi (2.3 M in hexanes, $0.58 \mathrm{~mL}, 1.33 \mathrm{mmol})$ was added dropwise to a stirred solution of $(R)-N$-benzyl- $N$-( $\alpha$-methylbenzyl)amine (289 mg, $1.37 \mathrm{mmol}$, >99:1 er) in THF $(2 \mathrm{~mL})$ at $-78{ }^{\circ} \mathrm{C}$ and the resultant mixture was stirred at $-78{ }^{\circ} \mathrm{C}$ for $30 \mathrm{~min}$. A solution of $\mathbf{1 6}(280 \mathrm{mg}$, $0.855 \mathrm{mmol},>99: 1 \mathrm{dr})$ in THF $(1 \mathrm{~mL})$ at $-78{ }^{\circ} \mathrm{C}$ was added and the resultant mixture was stirred at $-78{ }^{\circ} \mathrm{C}$ for $2 \mathrm{~h}$. Satd aq $\mathrm{NH}_{4} \mathrm{Cl}(0.5 \mathrm{~mL})$ was added and the reaction mixture was allowed to warm to $\mathrm{rt}$, then concentrated in vacuo. The residue was partitioned between $\mathrm{CH}_{2} \mathrm{Cl}_{2}(5 \mathrm{~mL})$ and $10 \%$ citric acid $(5 \mathrm{~mL})$, and the organic layer was washed with satd aq $\mathrm{NaHCO}_{3}(5 \mathrm{~mL})$ and brine $(5 \mathrm{~mL})$, then dried and concentrated in vacuo to give 21 in >95:5 dr. Purification via flash column chromatography (eluent $30-40{ }^{\circ} \mathrm{C}$ petrol/ $\left.\mathrm{Et}_{2} \mathrm{O} / \mathrm{NH}_{4} \mathrm{OH}, 100: 10: 1\right)$ gave 21 as a pale yellow oil $(449 \mathrm{mg}, 97 \%,>99: 1 \mathrm{dr}) ;[\alpha]_{\mathrm{D}}^{25}+23.8(c 1.0$ in $\left.\mathrm{CHCl}_{3}\right) ; v_{\max }(\mathrm{ATR}) 3025,2973,2931,2837(\mathrm{C}-\mathrm{H}), 1722(\mathrm{C}=\mathrm{O}), 1618(\mathrm{C}=\mathrm{C}) ; \delta_{\mathrm{H}}\left(400 \mathrm{MHz}, \mathrm{CDCl}_{3}\right) 1.28$ $\left(3 \mathrm{H}, \mathrm{d}, J\right.$ 6.8, C( $\left.\left(\alpha^{\prime}\right) M e\right), 1.29(3 \mathrm{H}, \mathrm{d}, J 6.9, \mathrm{C}(\alpha) M e), 1.32\left(9 \mathrm{H}, \mathrm{s}, \mathrm{C} M e_{3}\right), 1.39-1.50\left(2 \mathrm{H}, \mathrm{m}, \mathrm{C}(4) H_{\mathrm{A}}\right.$, $\left.\mathrm{C}\left(5^{\prime}\right) H_{\mathrm{A}}\right), 1.90-2.00\left(1 \mathrm{H}, \mathrm{m}, \mathrm{C}(4) H_{\mathrm{B}}\right), 2.06\left(1 \mathrm{H}, \mathrm{dd}, J 14.1,7.7, \mathrm{C}(2) H_{\mathrm{A}}\right), 2.03-2.19\left(1 \mathrm{H}, \mathrm{m}, \mathrm{C}\left(5^{\prime}\right) H_{\mathrm{B}}\right), 2.18$ $\left(1 \mathrm{H}, \mathrm{dd}, J 14.1,5.4, \mathrm{C}(2) H_{\mathrm{B}}\right), 2.48\left(1 \mathrm{H}\right.$, app dd, $\left.J 13.8,5.3, \mathrm{C}\left(6^{\prime}\right) H_{\mathrm{A}}\right), 2.81(1 \mathrm{H}, \mathrm{ddd}, J 13.8,11.0,4.7$, $\left.\mathrm{C}\left(6^{\prime}\right) H_{\mathrm{B}}\right), 3.47-3.59\left(2 \mathrm{H}, \mathrm{m}, \mathrm{C}(3) H, \mathrm{C}\left(2^{\prime}\right) H\right), 3.58\left(1 \mathrm{H}, \mathrm{d}, J 14.7, \mathrm{NCH}_{\mathrm{A}} \mathrm{H}_{\mathrm{B}} \mathrm{Ph}\right), 3.83(1 \mathrm{H}, \mathrm{d}, J 14.7$, $\left.\mathrm{NCH}_{\mathrm{A}} H_{\mathrm{B}} \mathrm{Ph}\right), 3.84\left(1 \mathrm{H}, \mathrm{q}, J\right.$ 6.8, C( $\left.\left.\alpha^{\prime}\right) H\right), 3.92(1 \mathrm{H}, \mathrm{q}, J 6.9, \mathrm{C}(\alpha) H), 5.40-5.47\left(1 \mathrm{H}, \mathrm{m}, \mathrm{C}\left(3^{\prime}\right) H\right), 5.71-5.77$ $\left(1 \mathrm{H}, \mathrm{m}, \mathrm{C}\left(4^{\prime}\right) H\right), 7.17-7.43(15 \mathrm{H}, \mathrm{m}, P h) ; \delta_{\mathrm{C}}\left(100 \mathrm{MHz}, \mathrm{CDCl}_{3}\right) 20.5(\mathrm{C}(\alpha) M e), 20.9\left(C\left(5^{\prime}\right)\right), 22.9$ $\left(\mathrm{C}\left(\alpha^{\prime}\right) M e\right), 28.2\left(\mathrm{CMe}_{3}\right), 38.3,38.5(C(2), C(4)), 40.3\left(C\left(6^{\prime}\right)\right), 50.4\left(\mathrm{NCH}_{2} \mathrm{Ph}\right), 50.9\left(C\left(2^{\prime}\right)\right), 52.7(C(3)), 58.2$ $\left(C\left(\alpha^{\prime}\right)\right)$, $58.6(C(\alpha)), 80.1\left(C \mathrm{Me}_{3}\right), 125.7\left(C\left(4^{\prime}\right)\right), 126.6,126.8,127.0(p-P h), 127.6,128.2,128.2,128.3$, 128.3, $128.6(o, m-P h), 129.6\left(C\left(3^{\prime}\right)\right), 142.1,144.2,146.2(i-P h), 172.1(C(1)) ; m / z\left(\mathrm{ESI}^{+}\right) 539\left([\mathrm{M}+\mathrm{H}]^{+}\right.$, 100\%); HRMS (ESI $\left.{ }^{+}\right) \mathrm{m} / z:[\mathrm{M}+\mathrm{H}]^{+}$Calcd for $\mathrm{C}_{36} \mathrm{H}_{47} \mathrm{~N}_{2} \mathrm{O}_{2}{ }^{+}$539.3632; Found 539.3648.

tert-Butyl $\quad\left(3 S, 2 ' S, \alpha S, \alpha^{\prime} R\right)-3-\left[N\right.$-benzyl- $N$-( $\alpha$-methylbenzyl)amino]-4-[ $N\left(1^{\prime}\right)$-( $\alpha^{\prime}$-methylbenzyl)-

1',2',5',6'-tetrahydropyridin-2'-yl]butanoate 22. BuLi (2.3 M in hexanes, $0.64 \mathrm{~mL}, 1.47 \mathrm{mmol}$ ) was added dropwise to a stirred solution of $(S)-N$-benzyl- $N$-( $\alpha$-methylbenzyl)amine (318 mg, $1.51 \mathrm{mmol},>99: 1$ er) in THF $(2 \mathrm{~mL})$ at $-78{ }^{\circ} \mathrm{C}$ and the resultant mixture was stirred at $-78{ }^{\circ} \mathrm{C}$ for $30 \mathrm{~min}$. A solution of $16(308 \mathrm{mg}$, $0.941 \mathrm{mmol},>99: 1 \mathrm{dr})$ in $\mathrm{THF}(1 \mathrm{~mL})$ at $-78^{\circ} \mathrm{C}$ was added and the resultant mixture was stirred at $-78{ }^{\circ} \mathrm{C}$ for 2 h. Satd aq $\mathrm{NH}_{4} \mathrm{Cl}(0.5 \mathrm{~mL})$ was added and the reaction mixture was allowed to warm to $\mathrm{rt}$, then concentrated in vacuo. The residue was partitioned between $\mathrm{CH}_{2} \mathrm{Cl}_{2}(5 \mathrm{~mL})$ and $10 \%$ citric acid $(5 \mathrm{~mL})$, and 
the organic layer was washed with satd aq $\mathrm{NaHCO}_{3}(5 \mathrm{~mL})$ and brine $(5 \mathrm{~mL})$, then dried and concentrated in vacuo to give 22 in >95:5 dr. Purification via flash column chromatography (eluent $30-40{ }^{\circ} \mathrm{C}$ petrol/ $\left.\mathrm{Et}_{2} \mathrm{O} / \mathrm{NH}_{4} \mathrm{OH}, 100: 10: 1\right)$ gave 22 as a pale yellow oil $(476 \mathrm{mg}, 94 \%,>99: 1 \mathrm{dr}) ;[\alpha]_{\mathrm{D}}^{25}+68.1(c 1.0 \mathrm{in}$ $\left.\mathrm{CHCl}_{3}\right) ; v_{\max }(\mathrm{ATR})$ 3064, 3025, 2973, $2932(\mathrm{C}-\mathrm{H}), 1722(\mathrm{C}=\mathrm{O}), 1609(\mathrm{C}=\mathrm{C}) ; \delta_{\mathrm{H}}\left(400 \mathrm{MHz}, \mathrm{CDCl}_{3}\right) 1.28$ $\left(3 \mathrm{H}, \mathrm{d}, J\right.$ 6.6, C( $\left.\left(\alpha^{\prime}\right) M e\right), 1.40(3 \mathrm{H}, \mathrm{d}, J 6.9, \mathrm{C}(\alpha) M e), 1.44\left(9 \mathrm{H}, \mathrm{s}, \mathrm{C} M e_{3}\right), 1.55-1.73\left(3 \mathrm{H}, \mathrm{m}, \mathrm{C}(4) H_{2}\right.$, $\left.\mathrm{C}\left(5^{\prime}\right) H_{\mathrm{A}}\right), 2.03-2.14\left(1 \mathrm{H}, \mathrm{m}, \mathrm{C}\left(5^{\prime}\right) H_{\mathrm{B}}\right), 2.17\left(1 \mathrm{H}, \mathrm{dd}, J 14.4,6.6, \mathrm{C}(2) H_{\mathrm{A}}\right), 2.30\left(1 \mathrm{H}, \mathrm{dd}, J 14.4,6.6, \mathrm{C}(2) H_{\mathrm{B}}\right)$, $2.41\left(1 \mathrm{H}\right.$, app dd, $\left.J 13.7,3.5, \mathrm{C}\left(6^{\prime}\right) H_{\mathrm{A}}\right), 2.76\left(1 \mathrm{H}, \mathrm{ddd}, J 13.7,10.4,4.8, \mathrm{C}\left(6^{\prime}\right) H_{\mathrm{B}}\right), 3.28-3.35(1 \mathrm{H}, \mathrm{m}$, $\left.\mathrm{C}\left(2^{\prime}\right) H\right), 3.55-3.63(1 \mathrm{H}, \mathrm{m}, \mathrm{C}(3) H), 3.63\left(1 \mathrm{H}, \mathrm{d}, J 15.0, \mathrm{NCH}_{\mathrm{A}} \mathrm{H}_{\mathrm{B}} \mathrm{Ph}\right), 3.76\left(1 \mathrm{H}, \mathrm{d}, J 15.0, \mathrm{NCH}_{\mathrm{A}} H_{\mathrm{B}} \mathrm{Ph}\right), 3.82$ $\left(1 \mathrm{H}, \mathrm{q}, J\right.$ 6.6, C( $\left.\left(\alpha^{\prime}\right) H\right), 4.01(1 \mathrm{H}, \mathrm{q}, J 6.9, \mathrm{C}(\alpha) H), 5.55-5.61\left(1 \mathrm{H}, \mathrm{m}, \mathrm{C}\left(3^{\prime}\right) H\right), 5.71-5.77\left(1 \mathrm{H}, \mathrm{m}, \mathrm{C}\left(4^{\prime}\right) H\right)$, 7.17-7.39 (15H, m, Ph); $\delta_{\mathrm{C}}\left(100 \mathrm{MHz}, \mathrm{CDCl}_{3}\right) 19.6(\mathrm{C}(\alpha) M e), 21.5\left(C\left(5^{\prime}\right)\right), 22.5\left(\mathrm{C}\left(\alpha^{\prime}\right) M e\right), 28.3\left(\mathrm{CMe}_{3}\right)$, $36.6(C(4)), 39.8,39.9\left(C(2), C\left(6^{\prime}\right)\right), 49.8\left(\mathrm{NCH}_{2} \mathrm{Ph}\right), 52.9\left(C\left(2^{\prime}\right)\right), 54.3(C(3)), 58.1\left(C\left(\alpha^{\prime}\right)\right), 58.9(C(\alpha)), 80.0$ $\left(C \mathrm{Me}_{3}\right), 125.3\left(C\left(4^{\prime}\right)\right), 126.6,126.8,126.9(p-P h), 127.7,128.1,128.2,128.3,128.3,128.5(o, m-P h), 130.4$ $\left(C\left(3^{\prime}\right)\right), 141.9,143.9,145.7(i-P h), 172.3(C(1)) ; m / z\left(\mathrm{ESI}^{+}\right) 539\left([\mathrm{M}+\mathrm{H}]^{+}, 100 \%\right)$; HRMS $\left(\mathrm{ESI}^{+}\right) \mathrm{m} / z:[\mathrm{M}+$ $\mathrm{H}]^{+}$Calcd for $\mathrm{C}_{36} \mathrm{H}_{47} \mathrm{~N}_{2} \mathrm{O}_{2}{ }^{+}$539.3632; Found 539.3631.

\section{$\left(2 S, 2 ' S, \alpha R, \alpha^{\prime} R\right)-1-\left[N\left(1^{\prime}\right)-(\alpha-M e t h y l b e n z y l)-1 ', 2 ', 5 ', 6 '-t e t r a h y d r o p y r i d i n-2 '-y l\right]-2-[N-b e n z y l-N-$}

( $\boldsymbol{\alpha}^{\prime}$-methylbenzyl)amino]pent-4-ene 23. DIBAL-H (1.0 M in PhMe, $\left.0.20 \mathrm{~mL}, 0.20 \mathrm{mmol}\right)$ was added dropwise to a stirred solution of $21(100 \mathrm{mg}, 0.19 \mathrm{mmol},>99: 1 \mathrm{dr})$ in $\mathrm{PhMe}(2 \mathrm{~mL})$ at $-78{ }^{\circ} \mathrm{C}$ and the resultant mixture was stirred at $-78{ }^{\circ} \mathrm{C}$ for $1 \mathrm{~h}$. $\mathrm{MeOH}(38 \mu \mathrm{L}, 0.93 \mathrm{mmol})$ and $\mathrm{Ph}_{3} \mathrm{P}=\mathrm{CH}_{2}(0.31 \mathrm{M}$ in PhMe/THF, $6.58 \mathrm{~mL}, 2.04 \mathrm{mmol})^{24}$ were added sequentially, and the resultant mixture was allowed to warm to $\mathrm{rt}$ and stirred at $\mathrm{rt}$ for $16 \mathrm{~h}$, then concentrated in vacuo. The residue was dissolved in $\mathrm{Et}_{2} \mathrm{O}(10 \mathrm{~mL})$ and the resultant solution was filtered through a short plug of Celite ${ }^{\circledR}$ (eluent $\mathrm{Et}_{2} \mathrm{O}$ ) and concentrated in vacuo. Purification via flash column chromatography (eluent 30-40 ${ }^{\circ} \mathrm{C}$ petrol/ $/ \mathrm{Et}_{2} \mathrm{O} / \mathrm{NH}_{4} \mathrm{OH}, 100: 5: 1$ ) gave 23 as a colourless oil $(54 \mathrm{mg}, 62 \%,>99: 1 \mathrm{dr}) ;[\alpha]_{\mathrm{D}}^{25}+28.2\left(c 1.0\right.$ in $\left.\mathrm{CHCl}_{3}\right) ; v_{\max }(\mathrm{ATR}) 3358,2961(\mathrm{C}-\mathrm{H}), 1596$

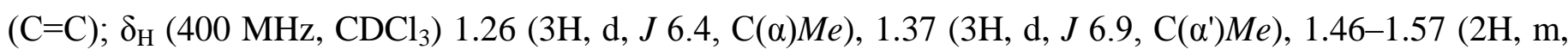
$\left.\mathrm{C}(1) H_{\mathrm{A}}, \mathrm{C}\left(5^{\prime}\right) H_{\mathrm{A}}\right), 1.89-2.01\left(2 \mathrm{H}, \mathrm{m}, \mathrm{C}(3) H_{\mathrm{A}}, \mathrm{C}(1) H_{\mathrm{B}}\right), 2.05-2.16\left(2 \mathrm{H}, \mathrm{m}, \mathrm{C}(3) H_{\mathrm{B}}, \mathrm{C}\left(5^{\prime}\right) H_{\mathrm{B}}\right), 2.49(1 \mathrm{H}$, app $\left.\mathrm{dd}, J 14.0,5.2, \mathrm{C}\left(6^{\prime}\right) H_{\mathrm{A}}\right), 2.86\left(1 \mathrm{H}, \mathrm{ddd}, J 14.0,11.2,4.6, \mathrm{C}\left(6^{\prime}\right) H_{\mathrm{B}}\right), 3.12(1 \mathrm{H}$, app quintet, $J$ 6.4, C(2)H), 3.38-3.46 (1H, m, C(2')H), $3.73\left(1 \mathrm{H}, \mathrm{d}, J 14.7, \mathrm{NCH}_{\mathrm{A}} \mathrm{H}_{\mathrm{B}} \mathrm{Ph}\right), 3.82(1 \mathrm{H}, \mathrm{q}, J$ 6.4, $\mathrm{C}(\alpha) H), 3.84(1 \mathrm{H}, \mathrm{d}, J 14.7$, $\left.\mathrm{NCH}_{\mathrm{A}} H_{\mathrm{B}} \mathrm{Ph}\right), 3.96\left(1 \mathrm{H}, \mathrm{q}, J\right.$ 6.9, C( $\left.\left.\alpha^{\prime}\right) H\right), 4.85-4.93\left(2 \mathrm{H}, \mathrm{m}, \mathrm{C}(5) H_{2}\right), 5.46-5.52\left(1 \mathrm{H}, \mathrm{m}, \mathrm{C}\left(3^{\prime}\right) H\right), 5.59(1 \mathrm{H}$, ddt, $J$ 17.0, 10.2, 7.0, C(4)H), 5.72-5.79 (1H, m, C(4')H), 7.18-7.34 (11H, m, Ph), 7.36-7.41 (2H, m, Ph), 7.42-7.46 (2H, m, Ph); $\delta_{\mathrm{C}}\left(100 \mathrm{MHz}, \mathrm{CDCl}_{3}\right) 17.9\left(\mathrm{C}\left(\alpha^{\prime}\right) M e\right), 20.6\left(C\left(5^{\prime}\right)\right), 22.9(\mathrm{C}(\alpha) M e), 36.4(C(3)), 37.9$ $(C(1)), 40.2\left(C\left(6^{\prime}\right)\right), 50.3\left(\mathrm{NCH}_{2} \mathrm{Ph}\right), 51.3\left(C\left(2^{\prime}\right)\right), 53.8(C(2)), 57.3\left(C\left(\alpha^{\prime}\right) \mathrm{Me}\right), 58.1(C(\alpha) \mathrm{Me}), 115.3(C(5))$, 
$125.5\left(C\left(4^{\prime}\right)\right), 126.5,126.6,126.6(p-P h), 127.4,127.9,128.1,128.2,128.2,128.5(o, m-P h), 130.0\left(C\left(3^{\prime}\right)\right)$, $\left.137.9(C(4)), 142.2,144.7,146.1(i-P h) ; m / z\left(\mathrm{ESI}^{+}\right) 465\left([\mathrm{M}+\mathrm{H}]^{+}, 100 \%\right) ; \mathrm{HRMS}_{(\mathrm{ESI}}{ }^{+}\right) m / z:[\mathrm{M}+\mathrm{H}]^{+}$ Calcd for $\mathrm{C}_{33} \mathrm{H}_{41} \mathrm{~N}_{2}{ }^{+}$465.3264; Found 465.3258.

$\left(2 S, 2 ' S, \alpha R, \alpha^{\prime} R, Z\right)-1-\left[N\left(1^{\prime}\right)-(\alpha-M e t h y l b e n z y l)-1 ', 2^{\prime}, 5^{\prime}, 6^{\prime}\right.$-tetrahydropyridin-2'-yl]-2-[N-benzyl- $N$ ( $\boldsymbol{\alpha}^{\prime}$-methylbenzyl)amino]hept-4-ene 24. DIBAL-H (1.0 M in PhMe, $\left.0.53 \mathrm{~mL}, 0.53 \mathrm{mmol}\right)$ was added dropwise to a stirred solution of $21(260 \mathrm{mg}, 0.483 \mathrm{mmol},>99: 1 \mathrm{dr})$ in $\mathrm{PhMe}(4 \mathrm{~mL})$ at $-78{ }^{\circ} \mathrm{C}$ and the resultant mixture was stirred at $-78^{\circ} \mathrm{C}$ for $1 \mathrm{~h}$. $\mathrm{MeOH}(98 \mu \mathrm{L}, 2.41 \mathrm{mmol})$ and $\mathrm{Ph}_{3} \mathrm{P}=\mathrm{CHCH}_{2} \mathrm{CH}_{3}(0.31 \mathrm{M}$ in $\mathrm{PhMe} / \mathrm{THF}, 15.6 \mathrm{~mL}, 4.83 \mathrm{mmol})^{25}$ were added sequentially, and the resultant mixture was allowed to warm to $\mathrm{rt}$ and stirred at $\mathrm{rt}$ for $16 \mathrm{~h}$, then concentrated in vacuo. The residue was dissolved in $\mathrm{Et}_{2} \mathrm{O}(20 \mathrm{~mL})$ and the

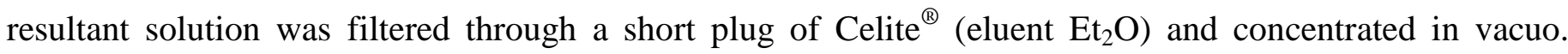
Purification via flash column chromatography (eluent $30-40{ }^{\circ} \mathrm{C}$ petrol/ $\mathrm{Et}_{2} \mathrm{O} / \mathrm{NH}_{4} \mathrm{OH}, 100: 5: 1$ ) gave 24 as a pale yellow oil $\left(148 \mathrm{mg}, 62 \%\right.$, >95:5 dr [(Z):(E)]); $[\alpha]_{\mathrm{D}}^{25}+36.5$ (c 1.0 in $\mathrm{CHCl}_{3}$ ); $v_{\max }$ (ATR) 3024, 2931, $2838(\mathrm{C}-\mathrm{H}), 1580,1493,1453(\mathrm{C}=\mathrm{C}) ; \delta_{\mathrm{H}}\left(400 \mathrm{MHz}, \mathrm{CDCl}_{3}\right) 0.91\left(3 \mathrm{H}, \mathrm{t}, J\right.$ 7.5, C(7)H $\left.H_{3}\right), 1.26(3 \mathrm{H}, \mathrm{d}, J$ 6.4, $\left.\mathrm{C}\left(\alpha^{\prime}\right) M e\right), 1.36(3 \mathrm{H}, \mathrm{d}, J 6.9, \mathrm{C}(\alpha) M e), 1.46-1.58\left(2 \mathrm{H}, \mathrm{m}, \mathrm{C}(1) H_{\mathrm{A}}, \mathrm{C}\left(5^{\prime}\right) H_{\mathrm{A}}\right), 1.89-2.16\left(6 \mathrm{H}, \mathrm{m}, \mathrm{C}(1) H_{\mathrm{B}}\right.$, $\left.\mathrm{C}(3) H_{2}, \mathrm{C}(6) H_{2}, \mathrm{C}\left(5^{\prime}\right) H_{\mathrm{B}}\right), 2.49\left(1 \mathrm{H}, \mathrm{dd}, J 14.0,5.2, \mathrm{C}\left(6^{\prime}\right) H_{\mathrm{A}}\right), 2.87\left(1 \mathrm{H}, \mathrm{ddd}, J 14.0,11.2,4.7, \mathrm{C}\left(6^{\prime}\right) H_{\mathrm{B}}\right)$, $3.06\left(1 \mathrm{H}\right.$, app quintet, $J$ 6.4, C(2)H), 3.40-3.47 (1H, m, C(2')H), $3.73\left(1 \mathrm{H}, \mathrm{d}, J 14.8, \mathrm{NCH}_{\mathrm{A}} \mathrm{H}_{\mathrm{B}} \mathrm{Ph}\right), 3.81(1 \mathrm{H}$, q, $J$ 6.4, C( $\left.\left(\alpha^{\prime}\right) H\right), 3.86\left(1 \mathrm{H}, \mathrm{d}, J 14.8, \mathrm{NCH}_{\mathrm{A}} H_{\mathrm{B}} \mathrm{Ph}\right), 3.98(1 \mathrm{H}, \mathrm{q}, J 6.9, \mathrm{C}(\alpha) H), 5.10-5.19(1 \mathrm{H}, \mathrm{m}, \mathrm{C}(4) H)$, 5.25-5.33 (1H, m, C(5)H), 5.44-5.50 (1H, m, C(3')H), 5.71-5.78 (1H, m, C(4')H), 7.19-7.35 (11H, m, Ph), 7.37-7.41 (2H, m, Ph), 7.42-7.46 (2H, m, Ph); $\delta_{\mathrm{C}}\left(100 \mathrm{MHz}, \mathrm{CDCl}_{3}\right) 14.3(C(7)), 18.5(\mathrm{C}(\alpha) M e), 20.8$ $\left(C\left(5^{\prime}\right)\right), 20.9(C(6)), 23.0\left(\mathrm{C}\left(\alpha^{\prime}\right) M e\right), 29.6(C(3)), 38.3(C(1)), 40.3\left(C\left(6^{\prime}\right)\right), 50.5\left(\mathrm{NCH}_{2} \mathrm{Ph}\right), 51.6\left(C\left(2^{\prime}\right)\right), 54.5$ $(C(2)), 57.7(C(\alpha)), 58.2\left(C\left(\alpha^{\prime}\right)\right), 125.5\left(C\left(4^{\prime}\right)\right), 126.5,126.7,126.7(p-P h), 127.6(o, m-P h), 128.0(C(4))$, 128.0, 128.2, 128.3, 128.3, $128.6(o, m-P h), 130.2\left(C\left(3^{\prime}\right)\right), 131.9(C(5)), 142.6,145.2,146.3(i-P h) ; m / z$ $\left(\mathrm{ESI}^{+}\right) 493\left([\mathrm{M}+\mathrm{H}]^{+}, 100 \%\right)$; HRMS $\left(\mathrm{ESI}^{+}\right) \mathrm{m} / z:[\mathrm{M}+\mathrm{H}]^{+}$Calcd for $\mathrm{C}_{35} \mathrm{H}_{45} \mathrm{~N}_{2}{ }^{+}$493.3577; Found 493.3568.

(2S,2'R)-1-(Piperidin-2'-yl)-2-aminopentane 25. Palladium black (15 mg, 20\% w/w) was added to a stirred, degassed solution of $23(75 \mathrm{mg}, 0.16 \mathrm{mmol})$ in $\mathrm{MeOH} / \mathrm{H}_{2} \mathrm{O} / \mathrm{AcOH}(10: 1: 1,2 \mathrm{~mL})$. The resultant mixture was stirred under an atmosphere of $\mathrm{H}_{2}(5 \mathrm{~atm})$ at $\mathrm{rt}$ for $48 \mathrm{~h}$. The reaction mixture was then filtered through a short plug of Celite ${ }^{\circledR}$ (eluent $\left.\mathrm{MeOH} / \mathrm{H}_{2} \mathrm{O} / \mathrm{AcOH}, 10: 1: 1\right)$ and concentrated in vacuo. Purification via flash column chromatography (eluent $\mathrm{CHCl}_{3} / \mathrm{MeOH} / \mathrm{NH}_{4} \mathrm{OH}$ 10:1:1) gave 25 as a colourless oil (20 mg, 73\%, >99:1 dr); $[\alpha]_{\mathrm{D}}^{25}-3.3\left(\right.$ c 1.0 in $\left.\mathrm{CHCl}_{3}\right) ; v_{\max }(\mathrm{ATR}) 3292(\mathrm{~N}-\mathrm{H}), 2930,2860(\mathrm{C}-\mathrm{H}) ; \delta_{\mathrm{H}}(400 \mathrm{MHz}$, $\left.\mathrm{CDCl}_{3}\right) 0.89\left(3 \mathrm{H}, \mathrm{t}, J 6.9, \mathrm{C}(5) H_{3}\right), 1.02-1.13\left(1 \mathrm{H}, \mathrm{m}, \mathrm{C}\left(3^{\prime}\right) H_{\mathrm{A}}\right), 1.17-1.46\left(8 \mathrm{H}, \mathrm{m}, \mathrm{C}(1) H_{2}, \mathrm{C}(3) H_{2}, \mathrm{C}(4) H_{2}\right.$, $\left.\mathrm{C}\left(4^{\prime}\right) H_{\mathrm{A}}, \mathrm{C}\left(5^{\prime}\right) H_{\mathrm{A}}\right), 1.53-1.68\left(2 \mathrm{H}, \mathrm{m}, \mathrm{C}\left(3^{\prime}\right) H_{\mathrm{B}}, \mathrm{C}\left(4^{\prime}\right) H_{\mathrm{B}}\right), 1.72-1.80\left(1 \mathrm{H}, \mathrm{m}, \mathrm{C}\left(5^{\prime}\right) H_{\mathrm{B}}\right), 2.55-2.66(2 \mathrm{H}, \mathrm{m}$, 
$\left.\mathrm{C}\left(2^{\prime}\right) H, \mathrm{C}\left(6^{\prime}\right) H_{\mathrm{A}}\right), 2.75-2.82(1 \mathrm{H}, \mathrm{m}, \mathrm{C}(2) H), 3.01-3.07\left(1 \mathrm{H}, \mathrm{m}, \mathrm{C}\left(6^{\prime}\right) H_{\mathrm{B}}\right) ; \delta_{\mathrm{C}}\left(100 \mathrm{MHz}, \mathrm{CDCl}_{3}\right) 14.3(C(5))$, $19.2(C(4)), 25.0\left(C\left(5^{\prime}\right)\right), 26.7\left(C\left(4^{\prime}\right)\right), 33.4\left(C\left(3^{\prime}\right)\right), 42.1(C(3)), 45.5(C(1)), 47.1\left(C\left(6^{\prime}\right)\right), 49.9(C(2)), 56.1$ $\left(C\left(2^{\prime}\right)\right) ; m / z\left(\mathrm{ESI}^{+}\right) 171\left([\mathrm{M}+\mathrm{H}]^{+}, 100 \%\right)$; HRMS $\left(\mathrm{ESI}^{+}\right) \mathrm{m} / z:[\mathrm{M}+\mathrm{H}]^{+}$Calcd for $\mathrm{C}_{10} \mathrm{H}_{23} \mathrm{~N}_{2}^{+}$171.1856; Found 171.1856.

(5S,6aR,11aS)-5-Propyldecahydro-5H-pyrido[1,2-c]pyrrolo[1,2-a]pyrimidine [(+)-tetraponerine

4] 27. Method A (from 25$): \mathrm{K}_{2} \mathrm{CO}_{3}(88 \mathrm{mg}, 0.63 \mathrm{mmol})$ and 4-bromobutanal (64 $\mathrm{mg}, 0.42 \mathrm{mmol}$ ) were added to a stirred solution of 25 (36 mg, $0.21 \mathrm{mmol},>99: 1 \mathrm{dr})$ in $\mathrm{CH}_{2} \mathrm{Cl}_{2}(2 \mathrm{~mL})$ at $\mathrm{rt}$, and the resultant mixture was stirred at $\mathrm{rt}$ for $2 \mathrm{~h} . \mathrm{H}_{2} \mathrm{O}(2 \mathrm{~mL})$ and satd aq $\mathrm{NaHCO}_{3}(10 \mathrm{~mL})$ were added, and the resultant mixture was extracted with $\mathrm{CH}_{2} \mathrm{Cl}_{2}(3 \times 5 \mathrm{~mL})$, then dried and concentrated in vacuo. Purification via flash column chromatography (eluent 30-40 ${ }^{\circ} \mathrm{C}$ petrol/ $\mathrm{Me}_{2} \mathrm{CO} / \mathrm{NH}_{4} \mathrm{OH}, 100: 10: 1$ ) gave (+)-tetraponerine 4 (27) as a colourless oil $(32 \mathrm{mg}, 68 \%$ from $\mathbf{2 5},>99: 1 \mathrm{dr}) ;[\alpha]_{\mathrm{D}}^{25}+90.8\left(c 1.0 \mathrm{in} \mathrm{CHCl}_{3}\right) ;\left\{\right.$ lit. ${ }^{4}$ for a sample isolated from the natural source $[\alpha]_{\mathrm{D}}^{20}+94\left(c 0.2\right.$ in $\left.\mathrm{CHCl}_{3}\right) ;$ lit. $^{9 \mathrm{~b}}[\alpha]_{\mathrm{D}}^{20}+96\left(c 2.0\right.$ in $\left.\left.\mathrm{CHCl}_{3}\right)\right\} ; v_{\max }$ (ATR) 2933, 2855, $2793(\mathrm{C}-\mathrm{H}) ;{ }^{26} \delta_{\mathrm{H}}\left(400 \mathrm{MHz}, \mathrm{C}_{6} \mathrm{D}_{6}\right) 0.89\left(3 \mathrm{H}, \mathrm{t}, J 7.1, \mathrm{C}\left(3^{\prime}\right) H_{3}\right), 1.13-1.29\left(2 \mathrm{H}, \mathrm{m}, \mathrm{C}(9) H_{\mathrm{A}}, \mathrm{C}\left(2^{\prime}\right) H_{\mathrm{A}}\right), 1.30-$ $1.56\left(9 \mathrm{H}, \mathrm{m}, \mathrm{C}(2) H_{\mathrm{A}}, \mathrm{C}(6) H_{2}, \mathrm{C}(7) H_{2}, \mathrm{C}(8) H_{\mathrm{A}}, \mathrm{C}\left(1^{\prime}\right) H_{2}, \mathrm{C}\left(2^{\prime}\right) H_{\mathrm{B}}\right), 1.59-1.77\left(7 \mathrm{H}, \mathrm{m}, \mathrm{C}(1) H_{2}, \mathrm{C}(2) H_{\mathrm{B}}\right.$, $\left.\mathrm{C}(6 \mathrm{a}) H, \mathrm{C}(8) H_{\mathrm{B}}, \mathrm{C}(9) H_{\mathrm{B}}, \mathrm{C}(10) H_{\mathrm{A}}\right), 2.02\left(1 \mathrm{H}, \mathrm{dd}, J 16.3,8.6, \mathrm{C}(3) H_{\mathrm{A}}\right), 2.11(1 \mathrm{H}, \mathrm{ddd}, J 14.6,7.4,3.6$, $\mathrm{C}(5) H), 2.31\left(1 \mathrm{H}, \mathrm{dd}, J\right.$ 7.8, 5.6, C(11a)H), 2.80-2.85 (1H, m, C(10)H $\left.H_{\mathrm{B}}\right), 3.13(1 \mathrm{H}$, app td, $J$ 8.6, 2.2, $\left.\mathrm{C}(3) H_{\mathrm{B}}\right) ; \delta_{\mathrm{C}}\left(100 \mathrm{MHz}, \mathrm{C}_{6} \mathrm{D}_{6}\right) 14.8\left(C\left(3^{\prime}\right)\right), 18.7\left(C\left(2^{\prime}\right)\right), 20.2(C(2)), 25.1(C(9)), 26.3(C(8)), 29.7(C(1))$, $33.0(C(7)), 36.9\left(C\left(1^{\prime}\right)\right), 38.0(C(6)), 49.0(C(3)), 51.6(C(10)), 61.1(C(5)), 62.7(C(6 a)), 85.6(C(11 \mathrm{a})) ; \mathrm{m} / \mathrm{z}$ $\left(\mathrm{ESI}^{+}\right) 223\left([\mathrm{M}+\mathrm{H}]^{+}, 100 \%\right)$; HRMS $\left(\mathrm{ESI}^{+}\right) \mathrm{m} / z:[\mathrm{M}+\mathrm{H}]^{+}$Calcd for $\mathrm{C}_{14} \mathrm{H}_{27} \mathrm{~N}_{2}{ }^{+}$223.2169; Found 223.2169. Method B (from 23): Palladium black (24 mg, 20\% w/w) was added to a stirred, degassed solution of 23 (121 $\mathrm{mg}, 0.26 \mathrm{mmol}$, >99:1 dr) in $\mathrm{MeOH} / \mathrm{H}_{2} \mathrm{O} / \mathrm{AcOH}(10: 1: 1,2 \mathrm{~mL})$. The resultant mixture was stirred under an atmosphere of $\mathrm{H}_{2}(5 \mathrm{~atm})$ at $\mathrm{rt}$ for $48 \mathrm{~h}$. The reaction mixture was then filtered through a short plug of Celite $^{\circledR}$ (eluent $\mathrm{MeOH} / \mathrm{H}_{2} \mathrm{O} / \mathrm{AcOH}, 10: 1: 1$ ), and concentrated in vacuo. The residue was dissolved in $\mathrm{CH}_{2} \mathrm{Cl}_{2}$ $(2 \mathrm{~mL})$, then $\mathrm{K}_{2} \mathrm{CO}_{3}(108 \mathrm{mg}, 0.78 \mathrm{mmol})$ and 4-bromobutanal $(79 \mathrm{mg}, 0.52 \mathrm{mmol})$ were added to the resultant solution. The reaction mixture was stirred at $\mathrm{rt}$ for $2 \mathrm{~h}$, then $\mathrm{H}_{2} \mathrm{O}(2 \mathrm{~mL})$ and satd aq $\mathrm{NaHCO}_{3}(10$ $\mathrm{mL})$ were added. The reaction mixture was then extracted with $\mathrm{CH}_{2} \mathrm{Cl}_{2}(3 \times 5 \mathrm{~mL})$ and the combined organic extracts were dried and concentrated in vacuo. Purification via flash column chromatography (eluent 30-40 ${ }^{\circ} \mathrm{C}$ petrol/ $\left.\mathrm{Me}_{2} \mathrm{CO} / \mathrm{NH}_{4} \mathrm{OH}, 100: 10: 1\right)$ gave (+)-tetraponerine $4(\mathbf{2 7})$ as a colourless oil (31 mg, 54\% from 23, $>99: 1 \mathrm{dr}) ;[\alpha]_{\mathrm{D}}^{25}+88.6\left(c 1.0\right.$ in $\left.\mathrm{CHCl}_{3}\right)$.

$(5 S, 6 a R, 11 a S)-5-P e n t y l d e c a h y d r o-5 H$-pyrido[1,2-c]pyrrolo[1,2-a]pyrimidine $[(+)$-tetraponerine

8] 28. Palladium black ( $26 \mathrm{mg}, 20 \% \mathrm{w} / \mathrm{w}$ ) was added to a stirred, degassed solution of 24 (130 mg, 0.26 
mmol, >95:5 dr) in $\mathrm{MeOH} / \mathrm{H}_{2} \mathrm{O} / \mathrm{AcOH}(10: 1: 1,2 \mathrm{~mL})$. The resultant mixture was stirred under an atmosphere of $\mathrm{H}_{2}(5 \mathrm{~atm})$ at $\mathrm{rt}$ for $48 \mathrm{~h}$. The reaction mixture was then filtered through a short plug of Celite $^{\circledR}$ (eluent $\mathrm{MeOH} / \mathrm{H}_{2} \mathrm{O} / \mathrm{AcOH}, 10: 1: 1$ ) and concentrated in vacuo. The residue was dissolved in $\mathrm{MeOH} / \mathrm{H}_{2} \mathrm{O} / \mathrm{AcOH}(10: 1: 1,2 \mathrm{~mL})$ and the resultant solution was degassed. $\mathrm{Pd}(\mathrm{OH})_{2} / \mathrm{C}(65 \mathrm{mg}, 50 \% \mathrm{w} / \mathrm{w})$ was added and the resultant mixture was stirred under an atmosphere of $\mathrm{H}_{2}(5 \mathrm{~atm})$ at $\mathrm{rt}$ for $24 \mathrm{~h}$. The reaction mixture was then filtered through a short plug of Celite ${ }^{\circledR}$ (eluent $\mathrm{MeOH} / \mathrm{H}_{2} \mathrm{O} / \mathrm{AcOH}, 10: 1: 1$ ) and concentrated in vacuo. The residue was dissolved in $\mathrm{CH}_{2} \mathrm{Cl}_{2}(2 \mathrm{~mL})$, then $\mathrm{K}_{2} \mathrm{CO}_{3}(110 \mathrm{mg}, 0.792 \mathrm{mmol})$ and 4-bromobutanal ( $80 \mathrm{mg}, 0.53 \mathrm{mmol}$ ) were added to the resultant solution. The reaction mixture was stirred at rt for $2 \mathrm{~h}$, then $\mathrm{H}_{2} \mathrm{O}(2 \mathrm{~mL})$ and satd aq $\mathrm{NaHCO}_{3}(10 \mathrm{~mL})$ were added. The reaction mixture was then extracted with $\mathrm{CH}_{2} \mathrm{Cl}_{2}(3 \times 5 \mathrm{~mL})$ and the combined organic extracts were dried and concentrated in vacuo. Purification via flash column chromatography (eluent $30-40{ }^{\circ} \mathrm{C}$ petrol/ $\mathrm{Me}_{2} \mathrm{CO} / \mathrm{NH}_{4} \mathrm{OH}, 100: 10: 1$ ) gave (+)tetraponerine $8(\mathbf{2 8})$ as a white solid $(44 \mathrm{mg}, 67 \%$ from $\mathbf{2 4},>99: 1 \mathrm{dr}) ; \mathrm{mp} 38-40{ }^{\circ} \mathrm{C} ;\left\{\right.$ lit. $\left.{ }^{5} \mathrm{mp} 40{ }^{\circ} \mathrm{C}\right\} ;[\alpha]_{\mathrm{D}}^{25}$ $+93.0\left(\right.$ c 1.0 in $\left.\mathrm{CHCl}_{3}\right) ;\left\{\right.$ lit. ${ }^{4}$ for a sample isolated from the natural source $[\alpha]_{\mathrm{D}}^{20}+102\left(c 0.2\right.$ in $\left.\mathrm{CHCl}_{3}\right)$; lit. ${ }^{9 \mathrm{~b}}$ $[\alpha]_{\mathrm{D}}^{20}+101\left(c 2.0\right.$ in $\left.\left.\mathrm{CHCl}_{3}\right)\right\} ; v_{\max }(\mathrm{ATR}) 2931,2857,2780(\mathrm{C}-\mathrm{H}) ;{ }^{26} \delta_{\mathrm{H}}\left(400 \mathrm{MHz}, \mathrm{C}_{6} \mathrm{D}_{6}\right) 0.90(3 \mathrm{H}, \mathrm{t}, J$ 7.0, $\left.\mathrm{C}\left(5^{\prime}\right) H_{3}\right), 1.16-1.67\left(17 \mathrm{H}, \mathrm{m}, \mathrm{C}(2) H_{\mathrm{A}}, \mathrm{C}(6) H_{2}, \mathrm{C}(7) H_{2}, \mathrm{C}(8) H_{2}, \mathrm{C}(9) H_{2}, \mathrm{C}\left(1^{\prime}\right) H_{2}, \mathrm{C}\left(2^{\prime}\right) H_{2}, \mathrm{C}\left(3^{\prime}\right) H_{2}, \mathrm{C}\left(4^{\prime}\right) H_{2}\right)$, 1.68-1.78 (5H, m, C(1)H $\left.H_{2}, \mathrm{C}(2) H_{\mathrm{B}}, \mathrm{C}(6 \mathrm{a}) H, \mathrm{C}(10) H_{\mathrm{A}}\right), 2.04\left(1 \mathrm{H}\right.$, app dd, $\left.J 16.3,8.5, \mathrm{C}(3) H_{\mathrm{A}}\right), 2.12(1 \mathrm{H}$, app ddd, $J$ 14.6, 7.3, 3.6, C(5)H), $2.31\left(1 \mathrm{H}, \mathrm{dd}, J\right.$ 7.9, 5.7, C(11a)H), 2.81-2.86 (1H, m, C(10)H $\left.H_{\mathrm{B}}\right), 3.15(1 \mathrm{H}$, app td, $J$ 8.5, 2.2, C(3)H $\left.H_{\mathrm{B}}\right) ; \delta_{\mathrm{C}}\left(100 \mathrm{MHz}, \mathrm{C}_{6} \mathrm{D}_{6}\right) 14.4\left(C\left(5^{\prime}\right)\right), 20.2(C(2)), 23.2\left(C\left(4^{\prime}\right)\right), 25.2(C(9)), 25.2\left(C\left(2^{\prime}\right)\right)$, $26.3(C(8)), 29.7(C(1)), 32.8\left(C\left(3^{\prime}\right)\right), 33.0(C(7)), 34.6\left(C\left(1^{\prime}\right)\right), 38.0(C(6)), 49.0(C(3)), 51.6(C(10)), 61.4$ $(C(5)), 62.7(C(6 a)), 85.6(C(11 \mathrm{a})) ; m / z\left(\mathrm{ESI}^{+}\right) 251\left([\mathrm{M}+\mathrm{H}]^{+}, 100 \%\right) ; \mathrm{HRMS}\left(\mathrm{ESI}^{+}\right) \mathrm{m} / z:[\mathrm{M}+\mathrm{H}]^{+}$Calcd for $\mathrm{C}_{16} \mathrm{H}_{31} \mathrm{~N}_{2}{ }^{+}$251.2482; Found 251.2472.

\section{$\left(2 R, 2^{\prime} S, \alpha S, \alpha^{\prime} R\right)-1-\left[N\left(1^{\prime}\right)-(\alpha-M e t h y l b e n z y l)-1 ', 2 ', 5 ', 6 '-t e t r a h y d r o p y r i d i n-2 '-y l\right]-2-[N-b e n z y l-N-$}

( $\boldsymbol{\alpha}^{\prime}$-methylbenzyl)amino]pent-4-ene 29. DIBAL-H (1.0 $\mathrm{M}$ in $\left.\mathrm{PhMe}, 0.78 \mathrm{~mL}, 0.78 \mathrm{mmol}\right)$ was added dropwise to a stirred solution of $22(383 \mathrm{mg}, 0.711 \mathrm{mmol},>99: 1 \mathrm{dr})$ in $\mathrm{PhMe}(6 \mathrm{~mL})$ at $-78{ }^{\circ} \mathrm{C}$ and the resultant mixture was stirred at $-78{ }^{\circ} \mathrm{C}$ for $1 \mathrm{~h}$. $\mathrm{MeOH}(144 \mu \mathrm{L}, 3.55 \mathrm{mmol})$ and $\mathrm{Ph}_{3} \mathrm{P}=\mathrm{CH}_{2}(0.31 \mathrm{M}$ in PhMe/THF, $22.9 \mathrm{~mL}, 7.11 \mathrm{mmol})^{24}$ were added sequentially, and the resultant mixture was allowed to warm to $\mathrm{rt}$ and stirred at $\mathrm{rt}$ for $16 \mathrm{~h}$, then concentrated in vacuo. The residue was dissolved in $\mathrm{Et}_{2} \mathrm{O}(30 \mathrm{~mL})$ and the resultant solution was filtered through a short plug of Celite ${ }^{\circledR}$ (eluent $\mathrm{Et}_{2} \mathrm{O}$ ) and concentrated in vacuo. Purification via flash column chromatography (eluent 30-40 ${ }^{\circ} \mathrm{C}$ petrol/Et ${ }_{2} \mathrm{O} / \mathrm{NH}_{4} \mathrm{OH}, 100: 5: 1$ ) gave 29 as a pale yellow oil $(180 \mathrm{mg}, 54 \%,>99: 1 \mathrm{dr}) ;[\alpha]_{\mathrm{D}}^{25}+52.5\left(c 1.0\right.$ in $\left.\mathrm{CHCl}_{3}\right) ; v_{\max }(\mathrm{ATR}) 3061,3025,2933,2836$ $(\mathrm{C}-\mathrm{H}), 1601,1492,1452(\mathrm{C}=\mathrm{C}) ; \delta_{\mathrm{H}}\left(400 \mathrm{MHz}, \mathrm{CDCl}_{3}\right) 1.26(3 \mathrm{H}, \mathrm{d}, J$ 6.6, $\mathrm{C}(\alpha) M e), 1.34(3 \mathrm{H}, \mathrm{d}, J$ 6.9, 
$\left.\mathrm{C}\left(\alpha^{\prime}\right) M e\right), 1.58-1.76\left(3 \mathrm{H}, \mathrm{d}, \mathrm{C}(1) H_{2}, \mathrm{C}\left(5^{\prime}\right) H_{\mathrm{A}}\right), 1.90-1.99\left(1 \mathrm{H}, \mathrm{m}, \mathrm{C}(3) H_{\mathrm{A}}\right), 2.00-2.15\left(2 \mathrm{H}, \mathrm{m}, \mathrm{C}(3) H_{\mathrm{B}}\right.$, $\left.\mathrm{C}\left(5^{\prime}\right) H_{\mathrm{B}}\right), 2.46\left(1 \mathrm{H}, \mathrm{ddd}, J 13.4,5.4,2.6, \mathrm{C}\left(6^{\prime}\right) H_{\mathrm{A}}\right), 2.83\left(1 \mathrm{H}, \mathrm{ddd}, J 13.4,9.9,4.7, \mathrm{C}\left(6^{\prime}\right) H_{\mathrm{B}}\right), 2.93(1 \mathrm{H}$, app quintet, $J$ 6.2, $\mathrm{C}(2) H), 3.24-3.30\left(1 \mathrm{H}, \mathrm{m}, \mathrm{C}\left(2^{\prime}\right) H\right), 3.73\left(1 \mathrm{H}, \mathrm{d}, J 14.8, \mathrm{NCH}_{\mathrm{A}} \mathrm{H}_{\mathrm{B}} \mathrm{Ph}\right), 3.81(1 \mathrm{H}, \mathrm{d}, J 14.8$, $\left.\mathrm{NCH}_{\mathrm{A}} H_{\mathrm{B}} \mathrm{Ph}\right), 3.82(1 \mathrm{H}, \mathrm{q}, J$ 6.6, C $(\alpha) H), 3.96\left(1 \mathrm{H}, \mathrm{q}, J\right.$ 6.9, C( $\left.\left.\alpha^{\prime}\right) H\right), 4.86-4.88\left(1 \mathrm{H}, \mathrm{m}, \mathrm{C}(5) H_{\mathrm{A}}\right), 4.89-4.92$ $\left(1 \mathrm{H}, \mathrm{m}, \mathrm{C}(5) H_{\mathrm{B}}\right), 5.54-5.65\left(2 \mathrm{H}, \mathrm{m}, \mathrm{C}(4) H, \mathrm{C}\left(3^{\prime}\right) H\right), 5.72-5.77\left(1 \mathrm{H}, \mathrm{m}, \mathrm{C}\left(4^{\prime}\right) H\right), 7.18-7.37(13 \mathrm{H}, \mathrm{m}, P h)$, 7.42-7.46 (2H, m, Ph); $\delta_{\mathrm{C}}\left(100 \mathrm{MHz}, \mathrm{CDCl}_{3}\right) 18.2\left(\mathrm{C}\left(\alpha^{\prime}\right) M e\right), 22.2\left(C\left(5^{\prime}\right)\right), 22.5(\mathrm{C}(\alpha) M e), 36.8(C(1)), 37.1$ $(C(3)), 40.1\left(C\left(6^{\prime}\right)\right), 50.1\left(\mathrm{NCH}_{2} \mathrm{Ph}\right), 53.1\left(C\left(2^{\prime}\right)\right), 55.1(C(2)), 57.6\left(C\left(\alpha^{\prime}\right)\right), 58.3(C(\alpha)), 115.2(C(5)), 125.2$ $\left(C\left(4^{\prime}\right)\right), 126.6,126.8,126.8(p-P h), 127.7,128.1,128.3,128.3,128.3,128.5(o, m-P h), 130.6\left(C\left(3^{\prime}\right)\right), 138.1$ (C(4)), 142.3, 144.4, $145.5(i-P h) ; m / z\left(\mathrm{ESI}^{+}\right) 465\left([\mathrm{M}+\mathrm{H}]^{+}, 100 \%\right) ; \mathrm{HRMS}\left(\mathrm{ESI}^{+}\right) \mathrm{m} / z:[\mathrm{M}+\mathrm{H}]^{+} \mathrm{Calcd}$ for $\mathrm{C}_{33} \mathrm{H}_{41} \mathrm{~N}_{2}{ }^{+}$465.3264; Found 465.3259.

$\left(2 R, 2^{\prime} S, \alpha S, \alpha^{\prime} R, Z\right)-1-\left[N\left(1^{\prime}\right)-(\alpha-M e t h y l b e n z y l)-1 ', 2 ', 5 ', 6\right.$ '-tetrahydropyridin-2'-yl]-2-[N-benzyl- $N$ -

( $\boldsymbol{\alpha}^{\prime}$-methylbenzyl)amino]hept-4-ene 30. DIBAL-H (1.0 M in PhMe, $\left.0.77 \mathrm{~mL}, 0.77 \mathrm{mmol}\right)$ was added dropwise to a stirred solution of $22(377 \mathrm{mg}, 0.700 \mathrm{mmol},>99: 1 \mathrm{dr})$ in $\mathrm{PhMe}(6 \mathrm{~mL})$ at $-78{ }^{\circ} \mathrm{C}$ and the resultant mixture was stirred at $-78{ }^{\circ} \mathrm{C}$ for $1 \mathrm{~h}$. $\mathrm{MeOH}(142 \mu \mathrm{L}, 3.50 \mathrm{mmol})$ and $\mathrm{Ph}_{3} \mathrm{P}=\mathrm{CHCH}_{2} \mathrm{CH}_{3}(0.31 \mathrm{M}$ in $\mathrm{PhMe} / \mathrm{THF}, 22.6 \mathrm{~mL}, 7.00 \mathrm{mmol})^{25}$ were added sequentially, and the resultant mixture was allowed to warm to $\mathrm{rt}$ and stirred at $\mathrm{rt}$ for $16 \mathrm{~h}$, then concentrated in vacuo. The residue was dissolved in $\mathrm{Et}_{2} \mathrm{O}(30 \mathrm{~mL})$ and the resultant solution was filtered through a short plug of Celite ${ }^{\circledR}$ (eluent $\mathrm{Et}_{2} \mathrm{O}$ ) and concentrated in vacuo. Purification via flash column chromatography (eluent $30-40{ }^{\circ} \mathrm{C}$ petrol/ $/ \mathrm{Et}_{2} \mathrm{O} / \mathrm{NH}_{4} \mathrm{OH}, 100: 5: 1$ ) gave 30 as a pale yellow oil $(162 \mathrm{mg}, 47 \%,>95: 5 \mathrm{dr}[(Z):(E)]) ;[\alpha]_{\mathrm{D}}^{25}+59.2\left(c 1.0\right.$ in $\left.\mathrm{CHCl}_{3}\right) ; v_{\max }(\mathrm{ATR}) 2970$ $(\mathrm{C}-\mathrm{H}), 1493,1453(\mathrm{C}=\mathrm{C}) ; \delta_{\mathrm{H}}\left(400 \mathrm{MHz}, \mathrm{CDCl}_{3}\right) 0.90(3 \mathrm{H}, \mathrm{t}, J$ 7.5, C(7)H $), 1.26(3 \mathrm{H}, \mathrm{d}, J$ 6.6, $\mathrm{C}(\alpha) M e)$, $1.34\left(3 \mathrm{H}, \mathrm{d}, J 6.9, \mathrm{C}\left(\alpha^{\prime}\right) M e\right), 1.60-1.80\left(3 \mathrm{H}, \mathrm{m}, \mathrm{C}(1) H_{2}, \mathrm{C}\left(5^{\prime}\right) H_{\mathrm{A}}\right), 1.91\left(2 \mathrm{H}\right.$, app quintet, $\left.J 7.5, \mathrm{C}(6) H_{2}\right), 1.97$ (2H, app t, $J$ 6.8, C(3)H $), 2.04-2.15\left(1 \mathrm{H}, \mathrm{m}, \mathrm{C}\left(5^{\prime}\right) H_{\mathrm{B}}\right), 2.47\left(1 \mathrm{H}, \mathrm{ddd}, J 13.1,5.3,3.1, \mathrm{C}\left(6^{\prime}\right) H_{\mathrm{A}}\right), 2.80-2.92$ $\left(2 \mathrm{H}, \mathrm{m}, \mathrm{C}(2) H, \mathrm{C}\left(6^{\prime}\right) H_{\mathrm{B}}\right), 3.20-3.26\left(1 \mathrm{H}, \mathrm{m}, \mathrm{C}\left(2^{\prime}\right) H\right), 3.73\left(1 \mathrm{H}, \mathrm{d}, J 14.8, \mathrm{NCH}_{\mathrm{A}} \mathrm{H}_{\mathrm{B}} \mathrm{Ph}\right), 3.81(1 \mathrm{H}, \mathrm{d}, J 14.8$, $\left.\mathrm{NCH}_{\mathrm{A}} H_{\mathrm{B}} \mathrm{Ph}\right), 3.83(1 \mathrm{H}, \mathrm{q}, J$ 6.6, C $(\alpha) H), 3.98\left(1 \mathrm{H}, \mathrm{q}, J\right.$ 6.9, $\left.\mathrm{C}\left(\alpha^{\prime}\right) H\right), 5.12-5.19(1 \mathrm{H}, \mathrm{m}, \mathrm{C}(4) H), 5.25-5.32$ $(1 \mathrm{H}, \mathrm{m}, \mathrm{C}(5) H), 5.56-5.61\left(1 \mathrm{H}, \mathrm{m}, \mathrm{C}\left(3^{\prime}\right) H\right), 5.71-5.77\left(1 \mathrm{H}, \mathrm{m}, \mathrm{C}\left(4^{\prime}\right) H\right), 7.17-7.44(15 \mathrm{H}, \mathrm{m}, P h) ; \delta_{\mathrm{C}}(100$ $\left.\mathrm{MHz}, \mathrm{CDCl}_{3}\right) 14.3(C(7)), 18.3\left(\mathrm{C}\left(\alpha^{\prime}\right) M e\right), 20.9(C(6)), 22.3(\mathrm{C}(\alpha) M e), 22.6\left(C\left(5^{\prime}\right)\right), 30.0(C(3)), 36.8(C(1))$, $40.2\left(C\left(6^{\prime}\right)\right), 50.0\left(\mathrm{NCH}_{2} \mathrm{Ph}\right), 53.2\left(C\left(2^{\prime}\right)\right), 55.5(C(2)), 57.6\left(C\left(\alpha^{\prime}\right)\right), 58.2(C(\alpha)), 125.1\left(C\left(4^{\prime}\right)\right)$, 126.6, 126.7, $126.8(p-P h), 127.8,128.0(o, m-P h), 128.1$ (C(4)), 128.2, 128.2, 128.3, $128.5(o, m-P h), 130.8\left(C\left(3^{\prime}\right)\right), 131.8$ $(C(5)), 142.4,144.7,145.2(i-P h) ; m / z\left(\mathrm{ESI}^{+}\right) 493\left([\mathrm{M}+\mathrm{H}]^{+}, 100 \%\right) ; \mathrm{HRMS}\left(\mathrm{ESI}^{+}\right) \mathrm{m} / z:[\mathrm{M}+\mathrm{H}]^{+}$Calcd for $\mathrm{C}_{35} \mathrm{H}_{45} \mathrm{~N}_{2}^{+}$493.3577; Found 493.3565. 
tetraponerine 3] 33. Palladium black ( $23 \mathrm{mg}, 20 \% \mathrm{w} / \mathrm{w}$ ) was added to a stirred, degassed solution of 29 (115 mg, $0.25 \mathrm{mmol}$, >99:1 dr) in $\mathrm{MeOH} / \mathrm{H}_{2} \mathrm{O} / \mathrm{AcOH}(10: 1: 1,2 \mathrm{~mL})$. The resultant mixture was stirred under an atmosphere of $\mathrm{H}_{2}(5 \mathrm{~atm})$ at $\mathrm{rt}$ for $48 \mathrm{~h}$. The reaction mixture was then filtered through a short plug of Celite ${ }^{\circledR}$ (eluent $\mathrm{MeOH} / \mathrm{H}_{2} \mathrm{O} / \mathrm{AcOH}, 10: 1: 1$ ) and concentrated in vacuo. The residue was dissolved in $\mathrm{CH}_{2} \mathrm{Cl}_{2}(2 \mathrm{~mL})$, then $\mathrm{K}_{2} \mathrm{CO}_{3}(102 \mathrm{mg}, 0.741 \mathrm{mmol})$ and 4-bromobutanal $(75 \mathrm{mg}, 0.49 \mathrm{mmol})$ were added to the resultant solution. The reaction mixture was stirred at $\mathrm{rt}$ for $2 \mathrm{~h}$, then $\mathrm{H}_{2} \mathrm{O}(2 \mathrm{~mL})$ and satd aq $\mathrm{NaHCO}_{3}$ $(10 \mathrm{~mL})$ were added. The reaction mixture was then extracted with $\mathrm{CH}_{2} \mathrm{Cl}_{2}(3 \times 5 \mathrm{~mL})$ and the combined organic extracts were dried and concentrated in vacuo. Purification via flash column chromatography (eluent 30-40 ${ }^{\circ} \mathrm{C}$ petrol/ $\left.\mathrm{Me}_{2} \mathrm{CO} / \mathrm{NH}_{4} \mathrm{OH}, 100: 10: 1\right)$ gave (+)-tetraponerine $3(33)$ as a colourless oil (33 mg, 60\% from 29, >99:1 dr); $[\alpha]_{\mathrm{D}}^{25}+30.9\left(c 1.0\right.$ in $\left.\mathrm{CHCl}_{3}\right)$; $\left\{\right.$ lit. $^{4}$ for a sample isolated from the natural source $[\alpha]_{\mathrm{D}}^{20}+27\left(c 0.07\right.$ in $\left.\mathrm{CHCl}_{3}\right) ;$ lit. $^{5}[\alpha]_{\mathrm{D}}^{20}+31$ ( 3.1 in $\left.\mathrm{CHCl}_{3}\right) ;$ lit. ${ }^{9 \mathrm{~d}}[\alpha]_{\mathrm{D}}^{20}+35\left(c 0.49\right.$ in $\left.\left.\mathrm{CHCl}_{3}\right)\right\} ; v_{\max }(\mathrm{ATR})$ 2954, 2928, 2859, $2802(\mathrm{C}-\mathrm{H}) ;{ }^{26} \delta_{\mathrm{H}}\left(400 \mathrm{MHz}, \mathrm{C}_{6} \mathrm{D}_{6}\right) 0.95(3 \mathrm{H}, \mathrm{t}, J$ 7.2, C(3')H $), 1.09$ (1H, app dt, $J$ 12.7, $\left.1.9, \mathrm{C}(6) H_{\mathrm{A}}\right), 1.15-1.28\left(1 \mathrm{H}, \mathrm{m}, \mathrm{C}(9) H_{\mathrm{A}}\right), 1.27-1.48\left(5 \mathrm{H}, \mathrm{m}, \mathrm{C}(7) H_{2}, \mathrm{C}\left(1^{\prime}\right) H_{\mathrm{A}}, \mathrm{C}\left(2^{\prime}\right) H_{2}\right), 1.48-1.84(9 \mathrm{H}, \mathrm{m}$, $\left.\mathrm{C}(1) H_{2}, \mathrm{C}(2) H_{2}, \mathrm{C}(8) H_{2}, \mathrm{C}(9) H_{\mathrm{B}}, \mathrm{C}(10) H_{\mathrm{A}}, \mathrm{C}\left(1^{\prime}\right) H_{\mathrm{B}}\right), 1.91\left(1 \mathrm{H}, \mathrm{ddd}, J 12.7,11.9,5.3, \mathrm{C}(6) H_{\mathrm{B}}\right), 1.99-2.07$ $(1 \mathrm{H}, \mathrm{m}, \mathrm{C}(6 \mathrm{a}) H), 2.72-2.86\left(3 \mathrm{H}, \mathrm{m}, \mathrm{C}(3) H_{\mathrm{A}}, \mathrm{C}(5) H, \mathrm{C}(10) H_{\mathrm{B}}\right), 3.17\left(1 \mathrm{H}\right.$, app q, $\left.J 7.5, \mathrm{C}(3) H_{\mathrm{B}}\right), 3.30(1 \mathrm{H}$, app t, $J 3.5, \mathrm{C}(11 \mathrm{a}) H) ; \delta_{\mathrm{C}}\left(100 \mathrm{MHz}, \mathrm{C}_{6} \mathrm{D}_{6}\right) 14.5\left(C\left(3^{\prime}\right)\right), 20.7\left(C\left(2^{\prime}\right)\right), 22.2(C(2)), 25.3(C(9)), 26.6(C(8))$, $30.6(C(1)), 32.2(C(6)), 33.4\left(C\left(1^{\prime}\right)\right), 34.3(C(7)), 50.6(C(3)), 51.0(C(10)), 53.0(C(5)), 56.7(C(6 a)), 75.4$ $(C(11 \mathrm{a})) ; \mathrm{m} / z\left(\mathrm{ESI}^{+}\right) 223\left([\mathrm{M}+\mathrm{H}]^{+}, 100 \%\right) ; \operatorname{HRMS}\left(\mathrm{ESI}^{+}\right) \mathrm{m} / z:[\mathrm{M}+\mathrm{H}]^{+}$Calcd for $\mathrm{C}_{14} \mathrm{H}_{27} \mathrm{~N}_{2}{ }^{+} 223.2169$; Found 223.2168.

$(5 R, 6 a R, 11 a S)-5-P e n t y l d e c a h y d r o-5 H$-pyrido[1,2-c]pyrrolo[1,2-a]pyrimidine $[(+)$-tetraponerine 7] 34. Palladium black ( $22 \mathrm{mg}, 20 \% \mathrm{w} / \mathrm{w}$ ) was added to a stirred, degassed solution of 30 (108 mg, 0.22 mmol, >95:5 dr) in $\mathrm{MeOH} / \mathrm{H}_{2} \mathrm{O} / \mathrm{AcOH}(10: 1: 1,2 \mathrm{~mL})$. The resultant mixture was stirred under an atmosphere of $\mathrm{H}_{2}(5 \mathrm{~atm})$ at $\mathrm{rt}$ for $48 \mathrm{~h}$. The reaction mixture was then filtered through a short plug of Celite ${ }^{\circledR}$ (eluent $\mathrm{MeOH} / \mathrm{H}_{2} \mathrm{O} / \mathrm{AcOH}, 10: 1: 1$ ) and concentrated in vacuo. The residue was dissolved in $\mathrm{CH}_{2} \mathrm{Cl}_{2}$ $(2 \mathrm{~mL})$, then $\mathrm{K}_{2} \mathrm{CO}_{3}(91 \mathrm{mg}, 0.66 \mathrm{mmol})$ and 4-bromobutanal $(66 \mathrm{mg}, 0.44 \mathrm{mmol})$ were added to the resultant solution. The reaction mixture was stirred at $\mathrm{rt}$ for $2 \mathrm{~h}$, then $\mathrm{H}_{2} \mathrm{O}(2 \mathrm{~mL})$ and satd aq $\mathrm{NaHCO}_{3}(10$ $\mathrm{mL})$ were added. The reaction mixture was then extracted with $\mathrm{CH}_{2} \mathrm{Cl}_{2}(3 \times 5 \mathrm{~mL})$ and the combined organic extracts were dried and concentrated in vacuo. Purification via flash column chromatography (eluent 30-40 ${ }^{\circ} \mathrm{C}$ petrol/ $\left.\mathrm{Me}_{2} \mathrm{CO} / \mathrm{NH}_{4} \mathrm{OH}, 100: 10: 1\right)$ gave (+)-tetraponerine 7 (34) as a colourless oil (24 mg, 44\% from 30, $>99: 1 \mathrm{dr}) ;[\alpha]_{\mathrm{D}}^{25}+30.4\left(c 0.8\right.$ in $\left.\mathrm{CHCl}_{3}\right) ;\left\{\right.$ lit. ${ }^{4}$ for a sample isolated from the natural source $[\alpha]_{\mathrm{D}}^{20}+30(c 0.22$ 
in $\mathrm{CHCl}_{3}$ ); lit. ${ }^{9 \mathrm{~b}}[\alpha]_{\mathrm{D}}^{20}+29.5\left(c 2.2\right.$ in $\left.\left.\mathrm{CHCl}_{3}\right)\right\} ; v_{\max }(\mathrm{ATR}) 2928,2856,2790(\mathrm{C}-\mathrm{H}) ;{ }^{26} \delta_{\mathrm{H}}\left(400 \mathrm{MHz}, \mathrm{C}_{6} \mathrm{D}_{6}\right)$ $0.93\left(3 \mathrm{H}, \mathrm{t}, J 6.9, \mathrm{C}\left(5^{\prime}\right) H_{3}\right), 1.13\left(1 \mathrm{H}\right.$, app dt, $\left.J 12.9,1.9, \mathrm{C}(6) H_{\mathrm{A}}\right), 1.17-1.86\left(19 \mathrm{H}, \mathrm{m}, \mathrm{C}(1) H_{2}, \mathrm{C}(2) H_{2}\right.$, $\left.\mathrm{C}(7) H_{2}, \mathrm{C}(8) H_{2}, \mathrm{C}(9) H_{2}, \mathrm{C}(10) H_{\mathrm{A}}, \mathrm{C}\left(1^{\prime}\right) H_{2}, \mathrm{C}\left(2^{\prime}\right) H_{2}, \mathrm{C}\left(3^{\prime}\right) H_{2}, \mathrm{C}\left(4^{\prime}\right) H_{2}\right), 1.93(1 \mathrm{H}, \mathrm{ddd}, J 12.9,11.9,5.4$, $\left.\mathrm{C}(6) H_{\mathrm{B}}\right), 2.02-2.09(1 \mathrm{H}, \mathrm{m}, \mathrm{C}(6 \mathrm{a}) H), 2.74-2.86\left(3 \mathrm{H}, \mathrm{m}, \mathrm{C}(3) H_{\mathrm{A}}, \mathrm{C}(5) H, \mathrm{C}(10) H_{\mathrm{B}}\right), 3.15-3.21(1 \mathrm{H}, \mathrm{m}$, $\left.\mathrm{C}(3) H_{\mathrm{B}}\right), 3.33(1 \mathrm{H}$, app t, $J 3.6, \mathrm{C}(11 \mathrm{a}) H) ; \delta_{\mathrm{C}}\left(100 \mathrm{MHz}, \mathrm{C}_{6} \mathrm{D}_{6}\right) 14.4\left(C\left(5^{\prime}\right)\right), 22.2(C(2)), 23.2\left(C\left(4^{\prime}\right)\right), 25.3$ $(C(8)), 26.6(C(9)), 27.4\left(C\left(2^{\prime}\right)\right), 30.6(C(1)), 31.1\left(C\left(1^{\prime}\right)\right), 32.3(C(6)), 32.5\left(C\left(3^{\prime}\right)\right), 34.3(C(7)), 50.7(C(3))$, $51.0(C(10)), 53.3(C(5)), 56.7(C(6 \mathrm{a})), 75.5(C(11 \mathrm{a})) ; \mathrm{m} / z\left(\mathrm{ESI}^{+}\right) 251\left([\mathrm{M}+\mathrm{H}]^{+}, 100 \%\right) ; \mathrm{HRMS}\left(\mathrm{ESI}^{+}\right) \mathrm{m} / z$ : $[\mathrm{M}+\mathrm{H}]^{+}$Calcd for $\mathrm{C}_{16} \mathrm{H}_{31} \mathrm{~N}_{2}{ }^{+}$251.2482; Found 251.2480.

tert-Butyl $(3 S, \alpha R, E)-3-[N$-allyl- $N$-( $\alpha$-methylbenzyl)amino]hex-4-enoate 35 . BuLi (2.30 $\mathrm{M}$ in hexanes, $14.0 \mathrm{~mL}, 32.3 \mathrm{mmol})$ was added dropwise to a stirred solution of $(R)-N$-benzyl- $N$ - $(\alpha-$ methylbenzyl)amine $(5.36 \mathrm{~g}, 33.3 \mathrm{mmol},>99: 1 \mathrm{er})$ in THF $(30 \mathrm{~mL})$ at $-78{ }^{\circ} \mathrm{C}$ and the resultant mixture was stirred at $-78{ }^{\circ} \mathrm{C}$ for $30 \mathrm{~min}$. A solution of $3(3.50 \mathrm{~g}, 20.8 \mathrm{mmol},>99: 1 \mathrm{dr})$ in $\mathrm{THF}(10 \mathrm{~mL})$ at $-78{ }^{\circ} \mathrm{C}$ was added dropwise via cannula and the resultant mixture was stirred at $-78{ }^{\circ} \mathrm{C}$ for $2 \mathrm{~h}$. $\mathrm{Satd}$ aq $\mathrm{NH}_{4} \mathrm{Cl}(10 \mathrm{~mL})$ was added and the reaction mixture was allowed to warm to $\mathrm{rt}$, then concentrated in vacuo. The residue was partitioned between $\mathrm{CH}_{2} \mathrm{Cl}_{2}(100 \mathrm{~mL})$ and $10 \%$ aq citric acid $(100 \mathrm{~mL})$, and the organic layer was washed with satd aq $\mathrm{NaHCO}_{3}(100 \mathrm{~mL})$ and brine $(100 \mathrm{~mL})$, then dried and concentrated in vacuo to give 35 in $>$ 95:5 dr. Purification via flash column chromatography (eluent 30-40 C petrol/EtOAc, 20:1) gave 35 as a pale yellow oil $(6.10 \mathrm{~g}, 89 \%,>99: 1 \mathrm{dr}) ;^{15 \mathrm{~b}}[\alpha]_{\mathrm{D}}^{25}-3.5\left(c 2.0\right.$ in $\left.\mathrm{CHCl}_{3}\right) ;\left\{\right.$ lit. ${ }^{15 \mathrm{c}}[\alpha]_{\mathrm{D}}^{20}-4.7\left(c 1.0\right.$ in $\left.\left.\mathrm{CHCl}_{3}\right)\right\}$; $\left.\delta_{\mathrm{H}}\left(400 \mathrm{MHz}, \mathrm{CDCl}_{3}\right) 1.36(3 \mathrm{H}, \mathrm{d}, J 6.8, \mathrm{C}(\alpha) M e), 1.40(9 \mathrm{H}, \mathrm{s}, \mathrm{CMe})_{3}\right), 1.69\left(3 \mathrm{H}, \mathrm{d}, J\right.$ 5.3, C(6)H $\left.H_{3}\right), 2.26(1 \mathrm{H}$, dd, $\left.J 14.2,8.4, \mathrm{C}(2) H_{\mathrm{A}}\right), 2.40\left(1 \mathrm{H}, \mathrm{dd}, J 14.2,6.4, \mathrm{C}(2) H_{\mathrm{B}}\right), 3.10-3.14\left(2 \mathrm{H}, \mathrm{m}, \mathrm{C}\left(1^{\prime}\right) H_{2}\right), 3.78-3.85(1 \mathrm{H}, \mathrm{m}$, $\mathrm{C}(3) H), 4.00(1 \mathrm{H}, \mathrm{q}, J$ 6.8, C $(\alpha) H), 4.99\left(1 \mathrm{H}, \mathrm{dd}, J 10.3,1.6, \mathrm{C}\left(3^{\prime}\right) H_{\mathrm{A}}\right), 5.07\left(1 \mathrm{H}, \mathrm{dd}, J 17.0,1.6, \mathrm{C}\left(3^{\prime}\right) H_{\mathrm{B}}\right)$, 5.43-5.58 (2H, m, C(4)H, C(5)H), $5.77(1 \mathrm{H}, \mathrm{ddt}, J$ 17.0, 10.3, 6.3, C(2')H), 7.17-7.22 (1H, m, Ph), 7.25$7.31(2 \mathrm{H}, \mathrm{m}, P h), 7.35-7.39(2 \mathrm{H}, \mathrm{m}, P h)$.

tert-Butyl (2'S, $\alpha R)-2$-[N(1')-( $\alpha$-methylbenzyl)-2',5'-dihydro-1H-pyrrol-2'-yl]acetate 36. Grubbs I catalyst (230 mg, $0.36 \mathrm{mmol}$ ) was added to a stirred, degassed solution of $\mathbf{3 5}$ (3.00 g, $9.11 \mathrm{mmol},>99: 1 \mathrm{dr})$ in anhydrous $\mathrm{CH}_{2} \mathrm{Cl}_{2}(\mathrm{EtOH}$ stabilised, $300 \mathrm{~mL})$ at $\mathrm{rt}$. The resultant mixture was stirred at $\mathrm{rt}$ for $16 \mathrm{~h}$, then concentrated in vacuo. The residue was dissolved in $\mathrm{CH}_{2} \mathrm{Cl}_{2}(80 \mathrm{~mL})$, then $\mathrm{P}\left(\mathrm{CH}_{2} \mathrm{OH}\right)_{3}(4.52 \mathrm{~g}, 36.4 \mathrm{mmol})$ and $\mathrm{Et}_{3} \mathrm{~N}(2.54 \mathrm{~mL}, 18.2 \mathrm{mmol})$ were added sequentially. The resultant mixture was stirred at rt for $5 \mathrm{~min}$, then excess silica gel $(10 \mathrm{~g})$ was added and the resultant mixture was stirred at $\mathrm{rt}$ for $12 \mathrm{~h}$ before being concentrated in vacuo. Purification via flash column chromatography (eluent $30-40{ }^{\circ} \mathrm{C}$ petrol/Et ${ }_{2} \mathrm{O} / \mathrm{NH}_{4} \mathrm{OH}$, 100:10:1) gave 36 as a pale yellow oil $(2.25 \mathrm{~g}, 86 \%,>99: 1 \mathrm{dr}){ }^{15 \mathrm{a}}[\alpha]_{\mathrm{D}}^{25}+99.0\left(c 1.0\right.$ in $\left.\mathrm{CHCl}_{3}\right)$; $\left\{\right.$ lit. ${ }^{15 \mathrm{~b}}$ for 
ent-36: $[\alpha]_{\mathrm{D}}^{24}-132.0\left(\right.$ c 0.99 in $\left.\left.\mathrm{CHCl}_{3}\right)\right\} ; \delta_{\mathrm{H}}\left(400 \mathrm{MHz}, \mathrm{CDCl}_{3}\right) 1.43(3 \mathrm{H}, \mathrm{d}, J 6.7, \mathrm{C}(\alpha) M e), 1.46(9 \mathrm{H}, \mathrm{s}$, $\left.\mathrm{CMe}_{3}\right), 2.33\left(1 \mathrm{H}, \mathrm{dd}, J 14.5,8.9, \mathrm{C}(2) H_{\mathrm{A}}\right), 2.59\left(1 \mathrm{H}, \mathrm{dd}, J 14.5,4.0, \mathrm{C}(2) H_{\mathrm{B}}\right), 3.34-3.41\left(1 \mathrm{H}, \mathrm{m}, \mathrm{C}\left(5^{\prime}\right) H_{\mathrm{A}}\right)$, 3.57-3.63 (1H, m, C(5') $\left.H_{\mathrm{B}}\right), 3.85(1 \mathrm{H}, \mathrm{q}, J$ 6.7, C $(\alpha) H), 4.10-4.18\left(1 \mathrm{H}, \mathrm{m}, \mathrm{C}\left(2^{\prime}\right) H\right), 5.68-5.76(2 \mathrm{H}, \mathrm{m}$, $\left.\mathrm{C}\left(3^{\prime}\right) H, \mathrm{C}\left(4^{\prime}\right) H\right), 7.19-7.34(5 \mathrm{H}, \mathrm{m}, P h)$.

tert-Butyl $(\boldsymbol{R}, \boldsymbol{R})-2-\left[N\left(\mathbf{1}^{\prime}\right)-\left(\boldsymbol{\alpha}\right.\right.$-methylbenzyl)pyrrolidin-2'-yl]acetate $37 . \mathrm{RhCl}\left(\mathrm{PPh}_{3}\right)_{3}(100 \mathrm{mg}, 0.11$ $\mathrm{mmol})$ was added to a degassed solution of $36(1.00 \mathrm{~g}, 3.48 \mathrm{mmol},>99: 1 \mathrm{dr})$ in EtOAc $(5 \mathrm{~mL})$ and the resultant mixture was stirred under $\mathrm{H}_{2}(2 \mathrm{~atm})$ at $\mathrm{rt}$ for $16 \mathrm{~h}$. The reaction mixture was then filtered through Celite $^{\circledR}$ (eluent EtOAc) and concentrated in vacuo. Purification via flash column chromatography (eluent 30$40{ }^{\circ} \mathrm{C}$ petrol/ $\left./ \mathrm{Et}_{2} \mathrm{O}, 10: 1\right)$ gave 37 as a colourless oil $(860 \mathrm{mg}, 86 \%,>99: 1 \mathrm{dr}){ }^{.15 \mathrm{~b}}[\alpha]_{\mathrm{D}}^{25}+57.0(c 1.0 \mathrm{in}$

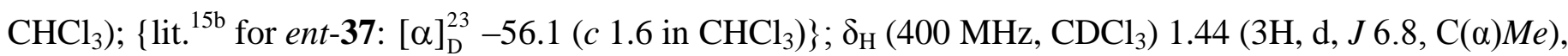
$1.44\left(9 \mathrm{H}, \mathrm{s}, \mathrm{C} M e_{3}\right), 1.55-1.64\left(2 \mathrm{H}, \mathrm{m}, \mathrm{C}\left(3^{\prime}\right) H_{\mathrm{A}}, \mathrm{C}\left(4^{\prime}\right) H_{\mathrm{A}}\right), 1.65-1.77\left(1 \mathrm{H}, \mathrm{m}, \mathrm{C}\left(4^{\prime}\right) H_{\mathrm{B}}\right), 1.79-1.89(1 \mathrm{H}, \mathrm{m}$, $\left.\mathrm{C}\left(3^{\prime}\right) H_{\mathrm{B}}\right), 2.18\left(1 \mathrm{H}, \mathrm{dd}, J 14.2,9.9, \mathrm{C}(2) H_{\mathrm{A}}\right), 2.39\left(1 \mathrm{H}, \mathrm{td}, J 8.8,6.9, \mathrm{C}\left(5^{\prime}\right) H_{\mathrm{A}}\right), 2.58(1 \mathrm{H}, \mathrm{dd}, J 14.2,3.5$, $\left.\mathrm{C}(2) H_{\mathrm{B}}\right), 2.76-2.82\left(1 \mathrm{H}, \mathrm{m}, \mathrm{C}\left(5^{\prime}\right) H_{\mathrm{B}}\right), 3.00-3.09\left(1 \mathrm{H}, \mathrm{m}, \mathrm{C}\left(2^{\prime}\right) H\right), 3.76(1 \mathrm{H}, \mathrm{q}, J 6.8, \mathrm{C}(\alpha) H), 7.21-7.35(5 \mathrm{H}$, $\mathrm{m}, P h)$.

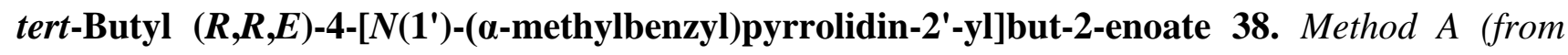
37): DIBAL-H (1.0 M in PhMe, $0.57 \mathrm{~mL}, 0.57 \mathrm{mmol})$ was added dropwise to a stirred solution of 37 (151 $\mathrm{mg}, 0.522 \mathrm{mmol},>99: 1 \mathrm{dr})$ in $\mathrm{PhMe}(2 \mathrm{~mL})$ at $-78{ }^{\circ} \mathrm{C}$ and the resultant mixture was stirred at $-78{ }^{\circ} \mathrm{C}$ for $1 \mathrm{~h}$. $\mathrm{MeOH}(0.11 \mathrm{~mL}, 2.61 \mathrm{mmol})$ and $\mathrm{Ph}_{3} \mathrm{P}=\mathrm{CHCO}_{2}{ }^{\mathrm{B}} \mathrm{Bu}(196 \mathrm{mg}, 0.522 \mathrm{mmol})$ were added sequentially, and the resultant mixture was allowed to warm to $\mathrm{rt}$ and stirred at $\mathrm{rt}$ for $16 \mathrm{~h}$. The reaction mixture was then concentrated in vacuo to give a 75:25 mixture of $\mathbf{3 8}$ and $\mathbf{3 9}$, respectively. Purification via flash column chromatography (eluent 30-40 ${ }^{\circ} \mathrm{C}$ petrol/ $\mathrm{Et}_{2} \mathrm{O} / \mathrm{NH}_{4} \mathrm{OH}, 100: 20: 1$ ) gave a 9:91 mixture of 38 and 39, respectively, as a pale yellow oil $(22 \mathrm{mg}, 13 \%)$. Data for 39: $\delta_{\mathrm{H}}\left(400 \mathrm{MHz}, \mathrm{CDCl}_{3}\right) 1.44(3 \mathrm{H}, \mathrm{d}, J 6.8$, $\mathrm{C}(\alpha) M e), 1.46-1.60\left(2 \mathrm{H}, \mathrm{m}, \mathrm{C}\left(3^{\prime}\right) H_{\mathrm{A}}, \mathrm{C}\left(4^{\prime}\right) H_{\mathrm{A}}\right), 1.50\left(9 \mathrm{H}, \mathrm{s}, \mathrm{C} M e_{3}\right), 1.65-1.77\left(2 \mathrm{H}, \mathrm{m}, \mathrm{C}\left(3^{\prime}\right) H_{\mathrm{B}}, \mathrm{C}\left(4^{\prime}\right) H_{\mathrm{B}}\right)$, $2.37\left(1 \mathrm{H}\right.$, app q, $J$ 8.2, C(5') $\left.H_{\mathrm{A}}\right), 2.65-2.79\left(2 \mathrm{H}, \mathrm{m}, \mathrm{C}(4) H_{\mathrm{A}}, \mathrm{C}\left(2^{\prime}\right) H\right), 2.84-2.90\left(1 \mathrm{H}, \mathrm{m}, \mathrm{C}\left(5^{\prime}\right) H_{\mathrm{B}}\right), 2.94-3.03$ $\left(1 \mathrm{H}, \mathrm{m}, \mathrm{C}(4) H_{\mathrm{B}}\right), 3.87(1 \mathrm{H}, \mathrm{q}, J$ 6.8, C $(\alpha) H), 5.75(1 \mathrm{H}, \mathrm{dt}, J 11.6,1.7, \mathrm{C}(2) H), 6.18(1 \mathrm{H}, \mathrm{dt}, J 11.6,7.2$, $\mathrm{C}(3) H), 7.21-7.37(5 \mathrm{H}, \mathrm{m}, \mathrm{Ph}) ; \delta_{\mathrm{C}}\left(100 \mathrm{MHz}, \mathrm{CDCl}_{3}\right) 22.0(\mathrm{C}(\alpha) M e), 22.6\left(C\left(4^{\prime}\right)\right), 28.4\left(\mathrm{CMe}_{3}\right), 29.9$ $\left(C\left(3^{\prime}\right)\right), 32.6(C(4)), 49.0\left(C\left(5^{\prime}\right)\right), 58.9\left(C\left(2^{\prime}\right)\right), 59.2(C(\alpha)), 80.1\left(C \mathrm{Me}_{3}\right), 122.5(C(2)), 126.9(p-P h), 128.1$, $128.2(o, m-P h), 142.4(i-P h), 146.2(C(3)), 166.2(C(1))$. Data for mixture: $v_{\max }(\mathrm{ATR}) 2972,2873(\mathrm{C}-\mathrm{H})$, $1713(\mathrm{C}=\mathrm{O}), 1651(\mathrm{C}=\mathrm{C}) ; \mathrm{m} / z\left(\mathrm{ESI}^{+}\right) 316\left([\mathrm{M}+\mathrm{H}]^{+}, 100 \%\right)$ HRMS $\left(\mathrm{ESI}^{+}\right) \mathrm{m} / z:[\mathrm{M}+\mathrm{H}]^{+}$Calcd for $\mathrm{C}_{20} \mathrm{H}_{30} \mathrm{NO}_{2}{ }^{+}$316.2271; Found 316.2269. Further elution (eluent 30-40 ${ }^{\circ} \mathrm{C}$ petrol/Et $2 \mathrm{O} / \mathrm{NH}_{4} \mathrm{OH}, 100: 20: 1$ ) gave an 86:14 mixture of $\mathbf{3 8}$ and $\mathbf{3 9}$, respectively, as a colourless oil (108 $\mathrm{mg}, 66 \%)$. 
Method B (from 36) - Step 1: $\mathrm{RhCl}\left(\mathrm{PPh}_{3}\right)_{3}(320 \mathrm{mg}, 0.348 \mathrm{mmol})$ was added to a degassed solution of 36 $(5.00 \mathrm{~g}, 17.4 \mathrm{mmol})$ in EtOAc $(100 \mathrm{~mL})$ and the resultant mixture was stirred under $\mathrm{H}_{2}(2 \mathrm{~atm})$ at $\mathrm{rt}$ for $16 \mathrm{~h}$. The reaction mixture was then filtered through Celite ${ }^{\circledR}$ (eluent EtOAc) and concentrated in vacuo to give 37. Method B (from 36) - Step 2: DIBAL-H (1.0 M in PhMe, 19.1 mL, $19.1 \mathrm{mmol})$ was added dropwise to a stirred solution of the residue of 37 from the previous step in $\mathrm{PhMe}(70 \mathrm{~mL})$ at $-78{ }^{\circ} \mathrm{C}$ and the resultant mixture was stirred at $-78{ }^{\circ} \mathrm{C}$ for $1 \mathrm{~h}$. $\mathrm{MeOH}(3.57 \mathrm{~mL}, 87.0 \mathrm{mmol})$ and $\mathrm{Ph}_{3} \mathrm{P}=\mathrm{CHCO}_{2}{ }^{\mathrm{t}} \mathrm{Bu}(6.55 \mathrm{~g}, 17.4$ mmol) were added sequentially, and the resultant mixture was allowed to warm to rt and stirred at rt for 16 h. The reaction mixture was then concentrated in vacuo to give a 75:25 mixture of $\mathbf{3 8}$ and 39, respectively. Purification via exhaustive flash column chromatography (eluent $30-40{ }^{\circ} \mathrm{C}$ petrol/ $\mathrm{Et}_{2} \mathrm{O} / \mathrm{NH}_{4} \mathrm{OH}, 100: 20: 1$ ) gave 38 as a pale yellow oil $\left(2.76 \mathrm{~g}, 50 \%\right.$ from 36, 98:2 dr); $[\alpha]_{\mathrm{D}}^{25}+82.7$ (c 1.0 in $\left.\mathrm{CHCl}_{3}\right) ; v_{\max }(\mathrm{ATR}) 2972$, $2873(\mathrm{C}-\mathrm{H}), 1713(\mathrm{C}=\mathrm{O}) ; \delta_{\mathrm{H}}\left(400 \mathrm{MHz}, \mathrm{CDCl}_{3}\right) 1.44(3 \mathrm{H}, \mathrm{d}, J$ 6.8, C( $\left.\alpha) M e\right), 1.48(9 \mathrm{H}, \mathrm{s}, \mathrm{CMe})_{3}, 1.48-1.81$ $\left(4 \mathrm{H}, \mathrm{m}, \mathrm{C}\left(3^{\prime}\right) H_{2}, \mathrm{C}\left(4^{\prime}\right) H_{2}\right), 2.18\left(1 \mathrm{H}\right.$, app dt, $\left.J 14.2,9.0, \mathrm{C}(4) H_{\mathrm{A}}\right), 2.40\left(1 \mathrm{H}\right.$, app td, $\left.J 8.7,7.2, \mathrm{C}\left(5^{\prime}\right) H_{\mathrm{A}}\right), 2.50$ $\left(1 \mathrm{H}\right.$, dddd, $J$ 14.2, 7.0, 3.3, 1.5, C $\left.(4) H_{\mathrm{B}}\right), 2.70-2.78\left(1 \mathrm{H}, \mathrm{m}, \mathrm{C}\left(2^{\prime}\right) H\right), 2.80-2.86\left(1 \mathrm{H}, \mathrm{m}, \mathrm{C}\left(5^{\prime}\right) H_{\mathrm{B}}\right), 3.80(1 \mathrm{H}$, q, $J$ 6.8, C( $\alpha) H), 5.76(1 \mathrm{H}, \mathrm{dt}, J 15.6,1.5, \mathrm{C}(2) H), 6.83(1 \mathrm{H}$, app dt, $J$ 15.6, 7.0, C(3)H), 7.21-7.34 (5H, m, $P h) ; \delta_{\mathrm{C}}\left(100 \mathrm{MHz}, \mathrm{CDCl}_{3}\right) 22.2(\mathrm{C}(\alpha) M e), 22.7\left(C\left(4^{\prime}\right)\right), 28.3\left(\mathrm{CMe}_{3}\right), 30.2\left(C\left(3^{\prime}\right)\right), 37.6(C(4)), 49.9\left(C\left(5^{\prime}\right)\right)$, $58.7\left(C\left(2^{\prime}\right)\right), 60.3(C(\alpha)), 80.2\left(C \mathrm{Me}_{3}\right), 124.4(C(2)), 127.0(p-P h), 128.0,128.2(o, m-P h), 142.9(i-P h)$, $145.9(C(3)), 166.1(C(1)) ; m / z\left(\mathrm{ESI}^{+}\right) 316\left([\mathrm{M}+\mathrm{H}]^{+}, 100 \%\right) ; \mathrm{HRMS}\left(\mathrm{ESI}^{+}\right) \mathrm{m} / z:[\mathrm{M}+\mathrm{H}]^{+}$Calcd for $\mathrm{C}_{20} \mathrm{H}_{30} \mathrm{NO}_{2}^{+}$316.2271; Found 316.2270.

tert-Butyl $\quad(R, R, R)-3-[N$-benzyl- $N$-isopropylamino]-4-[N(1')-( $\alpha$-methylbenzyl)pyrrolidin-2'yl]butanoate and tert-butyl $(3 S, 2 ' R, \alpha R)-3-[N$-benzyl- $N$-isopropylamino]-4-[N(1')-( $\alpha-$ methylbenzyl)pyrrolidin-2'-yl]butanoate 40 and 41. BuLi (2.3 $\mathrm{M}$ in hexanes, $0.11 \mathrm{~mL}, 0.253 \mathrm{mmol})$ was added dropwise to a stirred solution of $N$-benzyl- $N$-isopropylamine (38 mg, $0.25 \mathrm{mmol}$ ) in THF (0.5 mL) at $-78{ }^{\circ} \mathrm{C}$ and the resultant mixture was stirred at $-78{ }^{\circ} \mathrm{C}$ for $30 \mathrm{~min}$. A solution of $38(50 \mathrm{mg}, 0.159 \mathrm{mmol}$, 98:2 $\mathrm{dr}[(E):(Z)])$ in $\mathrm{THF}(0.5 \mathrm{~mL})$ at $-78^{\circ} \mathrm{C}$ was added via cannula and the resultant mixture was stirred at $-78{ }^{\circ} \mathrm{C}$ for $2 \mathrm{~h}$. Satd aq $\mathrm{NH}_{4} \mathrm{Cl}(0.2 \mathrm{~mL})$ was added and the reaction mixture was allowed to warm to $\mathrm{rt}$, then concentrated in vacuo. The residue was partitioned between $\mathrm{CH}_{2} \mathrm{Cl}_{2}(5 \mathrm{~mL})$ and $10 \%$ citric acid $(5 \mathrm{~mL})$, and the organic layer was washed with satd aq $\mathrm{NaHCO}_{3}(5 \mathrm{~mL})$ and brine $(5 \mathrm{~mL})$, then dried and concentrated in vacuo to give a 50:50 mixture of $\mathbf{4 0}$ and $\mathbf{4 1}$. Purification via flash column chromatography (eluent $30-40{ }^{\circ} \mathrm{C}$ petrol/Et $\left.{ }_{2} \mathrm{O} / \mathrm{NH}_{4} \mathrm{OH}, 100: 20: 1\right)$ gave a 50:50 mixture of $\mathbf{4 0}$ and $\mathbf{4 1}$ as a colourless oil (52 mg, 70\%). Data for 40: $\delta_{\mathrm{H}}\left(400 \mathrm{MHz}, \mathrm{CDCl}_{3}\right)$ 0.96-1.06 (6H, m, NCHMe $), 1.07-1.31\left(2 \mathrm{H}, \mathrm{m}, \mathrm{C}(4) H_{\mathrm{A}}, \mathrm{C}\left(3^{\prime}\right) H_{\mathrm{A}}\right), 1.34-1.41$ $\left(1 \mathrm{H}, \mathrm{m}, \mathrm{C}\left(3^{\prime}\right) H_{\mathrm{B}}\right), 1.39(3 \mathrm{H}, \mathrm{d}, J$ 6.7, C( $\left.\alpha) M e\right), 1.45(9 \mathrm{H}, \mathrm{s}, \mathrm{CMe}), 1.48-1.62\left(2 \mathrm{H}, \mathrm{m}, \mathrm{C}\left(4^{\prime}\right) H_{2}\right), 1.87(1 \mathrm{H}$, 
ddd, $J$ 13.5, 10.2, 3.8, C(4) $\left.H_{\mathrm{B}}\right), 2.10-2.18\left(1 \mathrm{H}, \mathrm{m}, \mathrm{C}(2) H_{\mathrm{A}}\right), 2.33-2.42\left(1 \mathrm{H}, \mathrm{m}, \mathrm{C}\left(5^{\prime}\right) H_{\mathrm{A}}\right), 2.64(1 \mathrm{H}, \mathrm{dd}, J$ 13.8, 3.8, C(2)HB $), 2.70\left(1 \mathrm{H}, \mathrm{ddd}, J 9.2,7.4,3.7, \mathrm{C}\left(5^{\prime}\right) H_{\mathrm{B}}\right), 2.87-2.97\left(2 \mathrm{H}, \mathrm{m}, \mathrm{C}\left(2^{\prime}\right) H, \mathrm{NCHMe}\right), 3.11(1 \mathrm{H}$, app tt, $J 14.5,3.8, \mathrm{C}(3) H), 3.43\left(1 \mathrm{H}, \mathrm{d}, J 14.1, \mathrm{NCH}_{\mathrm{A}} \mathrm{H}_{\mathrm{B}} \mathrm{Ph}\right), 3.70\left(1 \mathrm{H}, \mathrm{d}, J 14.1, \mathrm{NCH}_{\mathrm{A}} H_{\mathrm{B}} \mathrm{Ph}\right), 3.74(1 \mathrm{H}, \mathrm{q}, J$ 6.7, $\mathrm{C}(\alpha) H), 7.17-7.40(10 \mathrm{H}, \mathrm{m}, P h)$. Data for 41: $\delta_{\mathrm{H}}\left(400 \mathrm{MHz}, \mathrm{CDCl}_{3}\right)$ 0.96-1.06 (6H, m, NCHMe $), 1.45$ $(3 \mathrm{H}, \mathrm{d}, J 6.9, \mathrm{C}(\alpha) M e), 1.45\left(9 \mathrm{H}, \mathrm{s}, \mathrm{C} M e_{3}\right), 1.46-1.63\left(3 \mathrm{H}, \mathrm{m}, \mathrm{C}(4) H_{\mathrm{A}}, \mathrm{C}\left(3^{\prime}\right) H_{\mathrm{A}}, \mathrm{C}\left(4^{\prime}\right) H_{\mathrm{A}}\right), 1.67-1.87(3 \mathrm{H}$, m, C(4) $\left.H_{\mathrm{B}}, \mathrm{C}\left(3^{\prime}\right) H_{\mathrm{B}}, \mathrm{C}\left(4^{\prime}\right) H_{\mathrm{B}}\right), 2.10-2.18\left(1 \mathrm{H}, \mathrm{m}, \mathrm{C}(2) H_{\mathrm{A}}\right), 2.27-2.43\left(3 \mathrm{H}, \mathrm{m}, \mathrm{C}(2) H_{\mathrm{B}}, \mathrm{C}\left(2^{\prime}\right) H, \mathrm{C}\left(5^{\prime}\right) H_{\mathrm{A}}\right)$, 2.82-2.97 (2H, m, C(5') $\left.H_{\mathrm{B}}, \mathrm{NCHMe}\right), 3.22-3.29(1 \mathrm{H}, \mathrm{m}, \mathrm{C}(3) H), 3.62\left(1 \mathrm{H}, \mathrm{d}, J\right.$ 14.6, $\left.\mathrm{NCH}_{\mathrm{A}} \mathrm{H}_{\mathrm{B}} \mathrm{Ph}\right), 3.75$ $\left(1 \mathrm{H}, \mathrm{d}, J 14.6, \mathrm{NCH}_{\mathrm{A}} H_{\mathrm{B}} \mathrm{Ph}\right), 3.84(1 \mathrm{H}, \mathrm{q}, J 6.9, \mathrm{C}(\alpha) H), 7.17-7.40(10 \mathrm{H}, \mathrm{m}, P h)$. Data for mixture of 40 and 41: $v_{\max }(\mathrm{ATR}) 3026,2966,2932,2871(\mathrm{C}-\mathrm{H}), 1724(\mathrm{C}=\mathrm{O}) ; \delta_{\mathrm{C}}\left(100 \mathrm{MHz}, \mathrm{CDCl}_{3}\right)$ 18.8, 19.8, 21.4, 21.7, 22.5, 22.8, 22.9, 23.0, 28.2, 28.2, 29.6, 30.5, 37.4, 37.8, 39.2, 40.0, 46.8, 48.7, 48.9, 49.1, 49.3, 50.4, 52.6, 52.9, 57.5, 58.2, 59.4, 61.0, 80.1, 80.2, 126.6, 126.6, 126.7, 127.0, 127.9, 128.0, 128.0, 128.1, 128.1, 128.2, 128.6, 129.0, 141.1, 141.8, 142.1, 144.2, 172.4, 172.4; $\mathrm{m} / \mathrm{z}\left(\mathrm{ESI}^{+}\right) 465\left([\mathrm{M}+\mathrm{H}]^{+}, 100 \%\right) ; \mathrm{HRMS}\left(\mathrm{ESI}^{+}\right) \mathrm{m} / z$ : $[\mathrm{M}+\mathrm{H}]^{+}$Calcd for $\mathrm{C}_{30} \mathrm{H}_{45} \mathrm{~N}_{2} \mathrm{O}_{2}{ }^{+}$465.3476; Found 465.3475.

tert-Butyl

$(R, R, R, R)-3-[N-b e n z y l-N-(\alpha-m e t h y l b e n z y l) a m i n o]-4-\left[N\left(1^{\prime}\right)-\left(\alpha^{\prime}-\right.\right.$

methylbenzyl)pyrrolidin-2'-yl]butanoate 42. Method A: BuLi (2.3 M in hexanes, $0.85 \mathrm{~mL}, 1.96 \mathrm{mmol})$ was added dropwise to a stirred solution of $(R)$ - $N$-benzyl- $N$-( $\alpha$-methylbenzyl)amine (429 mg, $2.03 \mathrm{mmol}$, $>99: 1 \mathrm{er})$ in $\mathrm{THF}(3 \mathrm{~mL})$ at $-78{ }^{\circ} \mathrm{C}$ and the resultant mixture was stirred at $-78{ }^{\circ} \mathrm{C}$ for $30 \mathrm{~min}$. A solution of 38 (400 mg, $1.27 \mathrm{mmol}, 98: 2 \mathrm{dr}[(E):(Z)])$ in $\mathrm{THF}(2 \mathrm{~mL})$ at $-78{ }^{\circ} \mathrm{C}$ was added and the resultant mixture was stirred at $-78{ }^{\circ} \mathrm{C}$ for $2 \mathrm{~h}$. Satd aq $\mathrm{NH}_{4} \mathrm{Cl}(1 \mathrm{~mL})$ was added and the reaction mixture was allowed to warm to $\mathrm{rt}$, then concentrated in vacuo. The residue was partitioned between $\mathrm{CH}_{2} \mathrm{Cl}_{2}(10 \mathrm{~mL})$ and $10 \%$ citric acid (10 $\mathrm{mL})$, and the organic layer was washed with satd aq $\mathrm{NaHCO}_{3}(10 \mathrm{~mL})$ and brine $(10 \mathrm{~mL})$, then dried and concentrated in vacuo to give $\mathbf{4 2}$ in >95:5 dr. Purification via flash column chromatography (eluent 30-40 ${ }^{\circ} \mathrm{C}$ petrol/ $\left./ \mathrm{Et}_{2} \mathrm{O} / \mathrm{NH}_{4} \mathrm{OH}, 100: 20: 1\right)$ gave 42 as a pale yellow oil $(655 \mathrm{mg}, 98 \%,>99: 1 \mathrm{dr}) ;[\alpha]_{\mathrm{D}}^{25}+45.1(c 1.0$ in $\left.\mathrm{CHCl}_{3}\right) ; v_{\max }(\mathrm{ATR}) 3027,2971,2932,2872(\mathrm{C}-\mathrm{H}), 1723(\mathrm{C}=\mathrm{O}) ; \delta_{\mathrm{H}}\left(400 \mathrm{MHz}, \mathrm{CDCl}_{3}\right) 1.13(1 \mathrm{H}, \mathrm{ddd}, J$ 13.7, 10.9, 2.7, C(4)H $\left.H_{\mathrm{A}}\right), 1.32(3 \mathrm{H}, \mathrm{d}, J 7.0, \mathrm{C}(\alpha) M e), 1.38\left(3 \mathrm{H}\right.$, obsc d, C( $\left.\left.\alpha^{\prime}\right) M e\right), 1.40\left(9 \mathrm{H}, \mathrm{s}, \mathrm{CM} e_{3}\right), 1.42-$ $1.50\left(1 \mathrm{H}, \mathrm{m}, \mathrm{C}\left(3^{\prime}\right) H_{\mathrm{A}}\right), 1.53-1.74\left(3 \mathrm{H}, \mathrm{m}, \mathrm{C}\left(3^{\prime}\right) H_{\mathrm{B}}, \mathrm{C}\left(4^{\prime}\right) H_{2}\right), 1.75-1.83\left(2 \mathrm{H}, \mathrm{m}, \mathrm{C}(2) H_{2}\right), 1.92(1 \mathrm{H}, \mathrm{ddd}, J$ 13.7, 10.8, 2.7, C(4)HB $), 2.42\left(1 \mathrm{H}\right.$, app q, $J$ 8.1, C (5') $\left.H_{\mathrm{A}}\right), 2.73-2.79\left(1 \mathrm{H}, \mathrm{m}, \mathrm{C}\left(5^{\prime}\right) H_{\mathrm{B}}\right), 3.10-3.18(1 \mathrm{H}, \mathrm{m}$, $\left.\mathrm{C}\left(2^{\prime}\right) H\right), 3.34-3.41(1 \mathrm{H}, \mathrm{m}, \mathrm{C}(3) H), 3.44\left(1 \mathrm{H}, \mathrm{d}, J 14.8, \mathrm{NCH}_{\mathrm{A}} \mathrm{H}_{\mathrm{B}} \mathrm{Ph}\right), 3.73-3.81\left(2 \mathrm{H}, \mathrm{m}, \mathrm{C}(\alpha) H, \mathrm{C}\left(\alpha^{\prime}\right) H\right)$, $3.87\left(1 \mathrm{H}, \mathrm{d}, J\right.$ 14.8, $\left.\mathrm{NCH}_{\mathrm{A}} H_{\mathrm{B}} \mathrm{Ph}\right), 7.16-7.40(15 \mathrm{H}, \mathrm{m}, P h) ; \delta_{\mathrm{C}}\left(100 \mathrm{MHz}, \mathrm{CDCl}_{3}\right) 20.8(\mathrm{C}(\alpha) M e), 23.0$ $\left(\mathrm{C}\left(\alpha^{\prime}\right) M e\right), 23.1\left(C\left(4^{\prime}\right)\right), 28.2\left(\mathrm{CMe}_{3}\right), 29.8\left(C\left(3^{\prime}\right)\right), 37.9(C(2)), 38.8(C(4)), 50.4\left(\mathrm{NCH}_{2} \mathrm{Ph}\right), 50.7\left(C\left(5^{\prime}\right)\right)$, $51.8(C(3)), 57.1(C(\alpha)), 57.5\left(C\left(2^{\prime}\right)\right), 61.0\left(C\left(\alpha^{\prime}\right)\right), 80.1\left(C \mathrm{Me}_{3}\right), 126.7,126.8,127.1(p-P h), 127.9,128.2$, 
128.2, 128.3, 128.4, $128.5(o, m-P h), 141.1,142.5,144.2(i-P h), 172.1(C(1)) ; m / z\left(\mathrm{ESI}^{+}\right) 527\left([\mathrm{M}+\mathrm{H}]^{+}\right.$, 100\%); HRMS $\left(\mathrm{ESI}^{+}\right) \mathrm{m} / z:[\mathrm{M}+\mathrm{H}]^{+}$Calcd for $\mathrm{C}_{35} \mathrm{H}_{47} \mathrm{~N}_{2} \mathrm{O}_{2}{ }^{+}$527.3632; Found 527.3626.

Method B: BuLi (2.3 M in hexanes, $0.22 \mathrm{~mL}, 0.50 \mathrm{mmol}$ ) was added dropwise to a stirred solution of $(R)-N$ benzyl- $N$-( $\alpha$-methylbenzyl)amine $(109 \mathrm{mg}, 0.52 \mathrm{mmol},>99: 1 \mathrm{er})$ in THF $(2 \mathrm{~mL})$ at $-78^{\circ} \mathrm{C}$ and the resultant mixture was stirred at $-78{ }^{\circ} \mathrm{C}$ for $30 \mathrm{~min}$. A solution of $\mathbf{3 8}$ and $39(102 \mathrm{mg}, 0.32 \mathrm{mmol}, 86: 14 \mathrm{dr}[(E):(Z)])$ in THF $(1 \mathrm{~mL})$ at $-78{ }^{\circ} \mathrm{C}$ was added and the resultant mixture was stirred at $-78{ }^{\circ} \mathrm{C}$ for $2 \mathrm{~h}$. Satd aq $\mathrm{NH}_{4} \mathrm{Cl}(0.5$ $\mathrm{mL}$ ) was added and the reaction mixture was allowed to warm to rt, then concentrated in vacuo. The residue was partitioned between $\mathrm{CH}_{2} \mathrm{Cl}_{2}(5 \mathrm{~mL})$ and $10 \%$ citric acid $(5 \mathrm{~mL})$, and the organic layer was washed with satd aq $\mathrm{NaHCO}_{3}(5 \mathrm{~mL})$ and brine $(5 \mathrm{~mL})$, then dried and concentrated in vacuo to give an 86:14 mixture of 42 and 44, respectively. Purification via flash column chromatography (eluent $30-40 \quad{ }^{\circ} \mathrm{C}$ petrol/Et $\left.{ }_{2} \mathrm{O} / \mathrm{NH}_{4} \mathrm{OH}, 100: 20: 1\right)$ gave a 90:10 mixture of $\mathbf{4 2}$ and 44, respectively, as a pale yellow oil (143 mg). Data for 44: $\delta_{\mathrm{H}}\left(400 \mathrm{MHz}, \mathrm{CDCl}_{3}\right)$ [selected peaks] 2.28-2.36 $\left(1 \mathrm{H}, \mathrm{m}, \mathrm{C}\left(5^{\prime}\right) H_{\mathrm{A}}\right)$ 2.85-2.95 (2H, m, $\left.\mathrm{C}\left(2^{\prime}\right) H, \mathrm{C}\left(5^{\prime}\right) H_{\mathrm{B}}\right), 2.99\left(1 \mathrm{H}, \mathrm{dd}, J 15.9,6.9, \mathrm{C}(2) H_{\mathrm{A}}\right), 3.05\left(1 \mathrm{H}, \mathrm{dd}, J 15.9,6.9, \mathrm{C}(2) H_{\mathrm{B}}\right), 5.52(1 \mathrm{H}, \mathrm{dd}, J 15.5$, 8.3, C(4)H), $5.65(1 \mathrm{H}$, app dt, $J 15.5,6.9, \mathrm{C}(3) H) ; \mathrm{m} / z\left(\mathrm{ESI}^{+}\right) 527\left([\mathrm{M}(\mathbf{4 2})+\mathrm{H}]^{+}, 100 \%\right) ; 316\left([\mathrm{M}(\mathbf{4 4})+\mathrm{H}]^{+}\right.$, $32 \%)$.

tert-Butyl $\left(3 S, 2 ' R, \alpha S, \alpha^{\prime} R\right)-3-\left[N-\right.$ benzyl- $N-(\alpha-m e t h y l b e n z y l)$ amino]-4-[N(1')-( $\alpha^{\prime}-$ methylbenzyl)pyrrolidin-2'-yl]butanoate 43. BuLi (2.3 $\mathrm{M}$ in hexanes, $0.85 \mathrm{~mL}, 1.97 \mathrm{mmol})$ was added dropwise to a stirred solution of $(S)-N$-benzyl- $N$-( $\alpha$-methlbenzyl)amine (429 mg, $2.03 \mathrm{mmol}$, >99:1 er) in THF ( $3 \mathrm{~mL})$ at $-78{ }^{\circ} \mathrm{C}$ and the resultant mixture was stirred at $-78{ }^{\circ} \mathrm{C}$ for $30 \mathrm{~min}$. A solution of 38 (400 $\mathrm{mg}$, $1.27 \mathrm{mmol}, 98: 2 \mathrm{dr}[(E):(Z)])$ in THF $(2 \mathrm{~mL})$ at $-78{ }^{\circ} \mathrm{C}$ was added and the resultant mixture was stirred at $78{ }^{\circ} \mathrm{C}$ for $2 \mathrm{~h}$. Satd aq $\mathrm{NH}_{4} \mathrm{Cl}(1 \mathrm{~mL})$ was added and the reaction mixture was allowed to warm to $\mathrm{rt}$, then concentrated in vacuo. The residue was partitioned between $\mathrm{CH}_{2} \mathrm{Cl}_{2}(10 \mathrm{~mL})$ and $10 \%$ citric acid $(10 \mathrm{~mL})$, and the organic layer was washed with satd aq $\mathrm{NaHCO}_{3}(10 \mathrm{~mL})$ and brine $(10 \mathrm{~mL})$, then dried and concentrated in vacuo to give $\mathbf{4 3}$ in >95:5 dr. Purification via flash column chromatography (eluent 30-40 ${ }^{\circ} \mathrm{C}$ petrol/ $\left.\mathrm{Et}_{2} \mathrm{O} / \mathrm{NH}_{4} \mathrm{OH}, 100: 20: 1\right)$ gave 43 as a pale yellow oil $(615 \mathrm{mg}, 92 \%,>99: 1 \mathrm{dr}) ;[\alpha]_{\mathrm{D}}^{25}+72.8(c 1.0$ in $\left.\mathrm{CHCl}_{3}\right) ; v_{\max }(\mathrm{ATR}) 3027,2972,2932,2801(\mathrm{C}-\mathrm{H}), 1725(\mathrm{C}=\mathrm{O}) ; \delta_{\mathrm{H}}\left(400 \mathrm{MHz}, \mathrm{CDCl}_{3}\right) 1.34(3 \mathrm{H}, \mathrm{d}, J$ 6.9, $\mathrm{C}(\alpha) M e), 1.38\left(9 \mathrm{H}, \mathrm{s}, \mathrm{C} M e_{3}\right), 1.42\left(3 \mathrm{H}, \mathrm{d}, J\right.$ 6.9, C( $\left.\left.\alpha^{\prime}\right) M e\right), 1.41-1.47\left(1 \mathrm{H}, \mathrm{m}, \mathrm{C}\left(3^{\prime}\right) H_{\mathrm{A}}\right), 1.52-1.78(4 \mathrm{H}$, m, C(4) $\left.H_{\mathrm{A}}, \mathrm{C}\left(3^{\prime}\right) H_{\mathrm{B}}, \mathrm{C}\left(4^{\prime}\right) H_{2}\right), 1.84\left(1 \mathrm{H}, \mathrm{ddd}, J 13.0,9.8,2.5, \mathrm{C}(4) H_{\mathrm{B}}\right), 1.93\left(1 \mathrm{H}, \mathrm{dd}, J 15.1,6.6, \mathrm{C}(2) H_{\mathrm{A}}\right)$, $2.10\left(1 \mathrm{H}, \mathrm{dd}, J 15.1,5.8, \mathrm{C}(2) H_{\mathrm{B}}\right), 2.32-2.47\left(2 \mathrm{H}, \mathrm{m}, \mathrm{C}\left(2^{\prime}\right) H, \mathrm{C}\left(5^{\prime}\right) H_{\mathrm{A}}\right), 2.79-2.85\left(1 \mathrm{H}, \mathrm{m}, \mathrm{C}\left(5^{\prime}\right) H_{\mathrm{B}}\right), 3.31-$ $3.39(1 \mathrm{H}, \mathrm{m}, \mathrm{C}(3) H), 3.62\left(1 \mathrm{H}, \mathrm{d}, J 14.8, \mathrm{NCH}_{\mathrm{A}} \mathrm{H}_{\mathrm{B}} \mathrm{Ph}\right), 3.75\left(1 \mathrm{H}, \mathrm{d}, J 14.8, \mathrm{NCH}_{\mathrm{A}} H_{\mathrm{B}} \mathrm{Ph}\right), 3.80(1 \mathrm{H}, \mathrm{q}, J$ 6.9, $\left.\mathrm{C}\left(\alpha^{\prime}\right) H\right), 3.91(1 \mathrm{H}, \mathrm{q}, J$ 6.9, $\mathrm{C}(\alpha) H), 7.17-7.40(15 \mathrm{H}, \mathrm{m}, P h) ; \delta_{\mathrm{C}}\left(100 \mathrm{MHz}, \mathrm{CDCl}_{3}\right) 19.8(\mathrm{C}(\alpha) M e), 21.9$ 
(C( $\left.\left(\alpha^{\prime}\right) M e\right), 22.7\left(C\left(4^{\prime}\right)\right), 28.2\left(\mathrm{CMe}_{3}\right), 30.9\left(C\left(3^{\prime}\right)\right), 38.8(C(4)), 39.0(C(2)), 49.2\left(C\left(5^{\prime}\right)\right), 50.4\left(\mathrm{NCH}_{2} \mathrm{Ph}\right)$, $53.4(C(3)), 58.2\left(C\left(2^{\prime}\right)\right), 58.6(C(\alpha)), 59.8\left(C\left(\alpha^{\prime}\right)\right), 80.1\left(C \mathrm{Me}_{3}\right), 126.6,126.9,127.0(p-P h), 128.1,128.1$, 128.1, 128.2, 128.2, $128.5(o, m-P h), 141.8,142.5,144.1(i-P h), 172.1(C(1)) ; m / z\left(\mathrm{ESI}^{+}\right) 527\left([\mathrm{M}+\mathrm{H}]^{+}\right.$, 100\%); HRMS (ESI $\left.{ }^{+}\right) m / z:[\mathrm{M}+\mathrm{H}]^{+}$Calcd for $\mathrm{C}_{35} \mathrm{H}_{47} \mathrm{~N}_{2} \mathrm{O}_{2}{ }^{+}$527.3632; Found 527.3627.

$\left(2 S, 2 ' R, \alpha R, \alpha^{\prime} R\right)-1-\left[N\left(1^{\prime}\right)-(\alpha-M e t h y l b e n z y l) p y r r o l i d i n-2 '-y l\right]-2-\left[N-b e n z y l-N-\left(\alpha^{\prime}-\right.\right.$

methylbenzyl)amino]pent-4-ene 45. DIBAL-H (1.0 M in PhMe, $0.59 \mathrm{~mL}, 0.59 \mathrm{mmol})$ was added dropwise to a stirred solution of $42(284 \mathrm{mg}, 0.539 \mathrm{mmol},>99: 1 \mathrm{dr})$ in $\mathrm{PhMe}(5 \mathrm{~mL})$ at $-78{ }^{\circ} \mathrm{C}$ and the resultant mixture was stirred at $-78{ }^{\circ} \mathrm{C}$ for $1 \mathrm{~h}$. $\mathrm{MeOH}(109 \mu \mathrm{L}, 2.70 \mathrm{mmol})$ and $\mathrm{Ph}_{3} \mathrm{P}=\mathrm{CH}_{2}(0.31 \mathrm{M}$ suspension in PhMe/THF $17.4 \mathrm{~mL}, 5.39 \mathrm{mmol})^{24}$ were added sequentially, and the resultant mixture was allowed to warm to $\mathrm{rt}$ and stirred at $\mathrm{rt}$ for $16 \mathrm{~h}$, then concentrated in vacuo. The residue was dissolved in $\mathrm{Et}_{2} \mathrm{O}(40 \mathrm{~mL})$ and the

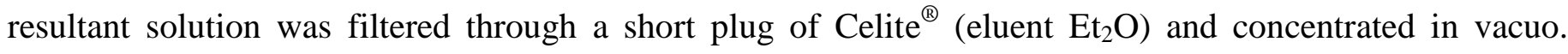
Purification via flash column chromatography (eluent 30-40 ${ }^{\circ} \mathrm{C}$ petrol/Et $\mathrm{E}_{2} \mathrm{O} / \mathrm{NH}_{4} \mathrm{OH}, 100: 10: 1$ ) gave 45 as a colourless oil (185 mg, 76\%, >99:1 dr); $[\alpha]_{\mathrm{D}}^{25}+76.9$ (c 1.0 in $\left.\mathrm{CHCl}_{3}\right) ; v_{\max }$ (ATR) 3026, 2970, 2933, 2870, $2803(\mathrm{C}-\mathrm{H}), 1638,1601,1493,1452(\mathrm{C}=\mathrm{C}) ; \delta_{\mathrm{H}}\left(400 \mathrm{MHz}, \mathrm{CDCl}_{3}\right) 1.21-1.31\left(2 \mathrm{H}, \mathrm{m}, \mathrm{C}(1) H_{\mathrm{A}}, \mathrm{C}\left(3^{\prime}\right) H_{\mathrm{A}}\right)$, $1.27\left(3 \mathrm{H}, \mathrm{d}, J\right.$ 7.0, C( $\left.\left(\alpha^{\prime}\right) M e\right), 1.37(3 \mathrm{H}, \mathrm{d}, J 6.8, \mathrm{C}(\alpha) M e), 1.48-1.72\left(4 \mathrm{H}, \mathrm{m}, \mathrm{C}(3) H_{\mathrm{A}}, \mathrm{C}\left(3^{\prime}\right) H_{\mathrm{B}}, \mathrm{C}\left(4^{\prime}\right) H_{2}\right)$, 1.72-1.80 (1H, m, C(3)H $\left.H_{\mathrm{B}}\right), 1.85\left(1 \mathrm{H}, \mathrm{ddd}, J 13.7,9.9,3.3, \mathrm{C}(1) H_{\mathrm{B}}\right), 2.36\left(1 \mathrm{H}\right.$, app q, $J$ 8.2, $\left.\mathrm{C}\left(5^{\prime}\right) H_{\mathrm{A}}\right), 2.73-$ $2.81\left(2 \mathrm{H}, \mathrm{m}, \mathrm{C}(2) H, \mathrm{C}\left(5^{\prime}\right) H_{\mathrm{B}}\right), 2.99-3.08\left(1 \mathrm{H}, \mathrm{m}, \mathrm{C}\left(2^{\prime}\right) H\right), 3.57\left(1 \mathrm{H}, \mathrm{d}, J 15.0, \mathrm{NCH}_{\mathrm{A}} \mathrm{H}_{\mathrm{B}} \mathrm{Ph}\right), 3.79(1 \mathrm{H}, \mathrm{q}, J$ 6.8, $\mathrm{C}(\alpha) H), 3.83\left(1 \mathrm{H}, \mathrm{q}, J 7.0, \mathrm{C}\left(\alpha^{\prime}\right) H\right), 3.92\left(1 \mathrm{H}, \mathrm{d}, J 15.0, \mathrm{NCH}_{\mathrm{A}} H_{\mathrm{B}} \mathrm{Ph}\right), 4.81\left(1 \mathrm{H}, \mathrm{d}, J 17.1, \mathrm{C}(5) H_{\mathrm{A}}\right), 4.87$ $\left(1 \mathrm{H}, \mathrm{d}, J 10.3, \mathrm{C}(5) H_{\mathrm{B}}\right), 5.46-5.57(1 \mathrm{H}, \mathrm{m}, \mathrm{C}(4) H), 7.16-7.36(13 \mathrm{H}, \mathrm{m}, P h), 7.39-7.43(2 \mathrm{H}, \mathrm{m}, P h) ; \delta_{\mathrm{C}}(100$ $\left.\mathrm{MHz}, \mathrm{CDCl}_{3}\right) 20.8\left(\mathrm{C}\left(\alpha^{\prime}\right) M e\right), 22.7(\mathrm{C}(\alpha) M e), 22.9\left(C\left(4^{\prime}\right)\right), 30.3\left(C\left(3^{\prime}\right)\right), 35.9(C(3)), 37.6(C(1)), 50.1$ $\left(C\left(5^{\prime}\right)\right), 50.4\left(\mathrm{NCH}_{2} \mathrm{Ph}\right), 55.0(C(2)), 57.4\left(C\left(\alpha^{\prime}\right)\right), 57.9\left(C\left(2^{\prime}\right)\right), 60.5(C(\alpha)), 116.1(C(5)), 126.6,126.8,127.0$ $(p-P h), 128.1,128.1,128.1,128.2,128.4,128.4(o, m-P h), 137.4(C(4)), 141.8,143.6,143.7(i-P h) ; m / z$ $\left(\mathrm{ESI}^{+}\right) 453\left([\mathrm{M}+\mathrm{H}]^{+}, 100 \%\right)$; HRMS $\left(\mathrm{ESI}^{+}\right) \mathrm{m} / z:[\mathrm{M}+\mathrm{H}]^{+}$Calcd for $\mathrm{C}_{32} \mathrm{H}_{41} \mathrm{~N}_{2}{ }^{+}$453.3264; Found 453.3259 .

$\left(2 S, 2^{\prime} R, \alpha R, \alpha^{\prime} R, Z\right)-1-\left[N\left(1^{\prime}\right)-(\alpha-M e t h y l b e n z y l) p y r r o l i d i n-2 '-y l\right]-2-\left[N-b e n z y l-N-\left(\alpha^{\prime}-\right.\right.$

methylbenzyl)amino]hept-4-ene 46. DIBAL-H (1.0 M in PhMe, $0.53 \mathrm{~mL}, 0.53 \mathrm{mmol})$ was added dropwise to a stirred solution of $42(255 \mathrm{mg}, 0.484 \mathrm{mmol},>99: 1 \mathrm{dr})$ in $\mathrm{PhMe}(5 \mathrm{~mL})$ at $-78{ }^{\circ} \mathrm{C}$ and the resultant mixture was stirred at $-78{ }^{\circ} \mathrm{C}$ for $1 \mathrm{~h}$. $\mathrm{MeOH}(98 \mu \mathrm{L}, 2.42 \mathrm{mmol})$ and $\mathrm{Ph}_{3} \mathrm{P}=\mathrm{CHCH}_{2} \mathrm{CH}_{3}(0.31 \mathrm{M}$ suspension in $\mathrm{PhMe} / \mathrm{THF} 15.6 \mathrm{~mL}, 4.84 \mathrm{mmol})^{25}$ were added sequentially, and the resultant mixture was allowed to warm to $\mathrm{rt}$ and stirred at $\mathrm{rt}$ for $16 \mathrm{~h}$, then concentrated in vacuo. The residue was dissolved in $\mathrm{Et}_{2} \mathrm{O}(40 \mathrm{~mL})$ and the resultant solution was filtered through a short plug of Celite ${ }^{\circledR}$ (eluent $\mathrm{Et}_{2} \mathrm{O}$ ) and concentrated in vacuo. Purification via flash column chromatography (eluent $30-40{ }^{\circ} \mathrm{C}$ petrol/ $\mathrm{Et}_{2} \mathrm{O} / \mathrm{NH}_{4} \mathrm{OH}, 100: 10: 1$ ) gave 
46 as a colourless oil $(174 \mathrm{mg}, 75 \%$, >95:5 dr $[(Z):(E)]) ;[\alpha]_{\mathrm{D}}^{25}+78.7\left(c 1.0\right.$ in $\left.\mathrm{CHCl}_{3}\right)$; $v_{\max }$ (ATR) 3061, 3026, 2966, 2932, $2872(\mathrm{C}-\mathrm{H}), 1602,1493,1453(\mathrm{C}=\mathrm{C})$; $\delta_{\mathrm{H}}\left(400 \mathrm{MHz}, \mathrm{CDCl}_{3}\right) 0.90(3 \mathrm{H}, \mathrm{t}, J$ 7.5, C(7)H $)$, 1.16-1.30 (2H, m, C $\left.(1) H_{\mathrm{A}}, \mathrm{C}\left(3^{\prime}\right) H_{\mathrm{A}}\right), 1.27\left(3 \mathrm{H}, \mathrm{d}, J\right.$ 7.0, $\left.\mathrm{C}\left(\alpha^{\prime}\right) M e\right), 1.38(3 \mathrm{H}, \mathrm{d}, J 6.8, \mathrm{C}(\alpha) M e), 1.51-1.65$ $\left(3 \mathrm{H}, \mathrm{m}, \mathrm{C}\left(3^{\prime}\right) H_{\mathrm{B}}, \mathrm{C}\left(4^{\prime}\right) H_{2}\right), 1.72-1.95\left(5 \mathrm{H}, \mathrm{m}, \mathrm{C}(1) H_{\mathrm{B}}, \mathrm{C}(3) H_{2}, \mathrm{C}(6) H_{2}\right), 2.39\left(1 \mathrm{H}\right.$, app q, $J$ 7.9, C(5') $\left.H_{\mathrm{A}}\right)$, 2.69-2.81 (2H, m, C(2)H, C(5') H $\left.H_{\mathrm{B}}\right), 2.98-3.06\left(1 \mathrm{H}, \mathrm{m}, \mathrm{C}\left(2^{\prime}\right) H\right), 3.60\left(1 \mathrm{H}, \mathrm{d}, J 15.1, \mathrm{NCH}_{\mathrm{A}} \mathrm{H}_{\mathrm{B}} \mathrm{Ph}\right), 3.80(1 \mathrm{H}$, q, $J$ 6.8, C( $\alpha) H), 3.86\left(1 \mathrm{H}, \mathrm{q}, J\right.$ 7.0, C( $\left.\left.\alpha^{\prime}\right) H\right), 3.96\left(1 \mathrm{H}, \mathrm{d}, J 15.1, \mathrm{NCH}_{\mathrm{A}} H_{\mathrm{B}} \mathrm{Ph}\right), 5.03-5.12(1 \mathrm{H}, \mathrm{m}, \mathrm{C}(4) H)$, 5.24-5.32 (1H, m, C(5)H), 7.18-7.37 (13H, m, Ph), 7.40-7.43 (2H, m, Ph); $\delta_{\mathrm{C}}\left(100 \mathrm{MHz}, \mathrm{CDCl}_{3}\right) 14.3$ $(C(7)), 20.8(C(6)), 21.1\left(\mathrm{C}\left(\alpha^{\prime}\right) M e\right), 22.6(\mathrm{C}(\alpha) M e), 22.8\left(C\left(4^{\prime}\right)\right), 28.7(C(3)), 30.2\left(C\left(3^{\prime}\right)\right), 37.5(C(1)), 50.0$ $\left(C\left(5^{\prime}\right)\right), 50.5\left(\mathrm{NCH}_{2} \mathrm{Ph}\right), 55.8(C(2)), 57.8\left(C\left(\alpha^{\prime}\right)\right), 57.8\left(C\left(2^{\prime}\right)\right), 60.3(C(\alpha)), 126.5,126.8,126.9(p-P h), 127.5$ (C(4)), 128.0, 128.1, 128.1, 128.2, 128.3, 128.4 (o,m-Ph), 132.5 (C(5)), 142.1, 143.7, $144.3(i-P h) ; \mathrm{m} / \mathrm{z}$ $\left(\mathrm{ESI}^{+}\right) 481\left([\mathrm{M}+\mathrm{H}]^{+}, 100 \%\right)$; HRMS $\left(\mathrm{ESI}^{+}\right) \mathrm{m} / z:[\mathrm{M}+\mathrm{H}]^{+}$Calcd for $\mathrm{C}_{34} \mathrm{H}_{45} \mathrm{~N}_{2}{ }^{+}$481.3577; Found 481.3574.

$(5 S, 6 \mathrm{a} R, 10 \mathrm{a} S)$-5-Propyldecahydrodipyrrolo[1,2-a:1',2'-c]pyrimidine [(+)-tetraponerine 2$] 49$. Palladium black (37 mg, 20\% w/w) was added to a stirred, degassed solution of 45 (183 mg, $0.404 \mathrm{mmol}$, $>99: 1 \mathrm{dr})$ in $\mathrm{MeOH} / \mathrm{H}_{2} \mathrm{O} / \mathrm{AcOH}(10: 1: 1,2 \mathrm{~mL})$. The resultant mixture was stirred under an atmosphere of $\mathrm{H}_{2}$ $(5 \mathrm{~atm})$ at $\mathrm{rt}$ for $48 \mathrm{~h}$. The reaction mixture was then filtered through a short plug of Celite ${ }^{\circledR}$ (eluent $\left.\mathrm{MeOH} / \mathrm{H}_{2} \mathrm{O} / \mathrm{AcOH}, 10: 1: 1\right)$ and concentrated in vacuo. The residue was dissolved in $\mathrm{CH}_{2} \mathrm{Cl}_{2}(2 \mathrm{~mL})$, then $\mathrm{K}_{2} \mathrm{CO}_{3}(168 \mathrm{mg}, 1.21 \mathrm{mmol})$ and 4-bromobutanal $(123 \mathrm{mg}, 0.81 \mathrm{mmol})$ were added to the resultant solution. The reaction mixture was stirred at $\mathrm{rt}$ for $2 \mathrm{~h}$, then $\mathrm{H}_{2} \mathrm{O}(2 \mathrm{~mL})$ and satd aq $\mathrm{NaHCO}_{3}(10 \mathrm{~mL})$ were added. The reaction mixture was then extracted with $\mathrm{CH}_{2} \mathrm{Cl}_{2}(3 \times 5 \mathrm{~mL})$ and the combined organic extracts were dried and concentrated in vacuo. Purification via flash column chromatography (eluent $30-40{ }^{\circ} \mathrm{C}$ petrol/ $\left.\mathrm{Me}_{2} \mathrm{CO} / \mathrm{NH}_{4} \mathrm{OH}, 100: 10: 1\right)$ gave (+)-tetraponerine 2 (49) as a colourless oil (31 mg, 37\% from 45, >99:1 dr); $[\alpha]_{\mathrm{D}}^{25}+44.2\left(c \quad 1.0\right.$ in $\left.\mathrm{CHCl}_{3}\right) ;\left\{\right.$ lit. $^{5}[\alpha]_{\mathrm{D}}^{20}+36$ (c 1.79 in $\left.\mathrm{CHCl}_{3}\right)$; lit. ${ }^{6}[\alpha]_{\mathrm{D}}^{20}+47(c \quad 0.232$ in $\left.\left.\mathrm{CHCl}_{3}\right)\right\} ; v_{\max }(\mathrm{ATR}) 2957,2931,2871,2786(\mathrm{C}-\mathrm{H}) ;{ }^{26} \delta_{\mathrm{H}}\left(400 \mathrm{MHz}, \mathrm{C}_{6} \mathrm{D}_{6}\right) 0.92\left(3 \mathrm{H}, \mathrm{t}, J\right.$ 7.2, C(3') $\left.H_{3}\right)$, 1.27-1.83 (14H, m, C(1) $\left.H_{2}, \mathrm{C}(2) H_{2}, \mathrm{C}(6) H_{2}, \mathrm{C}(7) H_{2}, \mathrm{C}(8) H_{2}, \mathrm{C}\left(1^{\prime}\right) H_{2}, \mathrm{C}\left(2^{\prime}\right) H_{2}\right), 1.83-1.97\left(2 \mathrm{H}, \mathrm{m}, \mathrm{C}(9) H_{\mathrm{A}}\right.$, $\mathrm{C}(6 \mathrm{a}) H), 2.29-2.36\left(1 \mathrm{H}, \mathrm{m}, \mathrm{C}(3) H_{\mathrm{A}}\right), 2.39-2.46(1 \mathrm{H}, \mathrm{m}, \mathrm{C}(5) H), 2.86(1 \mathrm{H}$, app t, $J$ 5.3, C(10a)H), $2.93(1 \mathrm{H}$, app td, $J$ 8.5, 2.5, C(9) $\left.H_{\mathrm{B}}\right), 3.01-3.07\left(1 \mathrm{H}, \mathrm{m}, \mathrm{C}(3) H_{\mathrm{B}}\right)$; $\delta_{\mathrm{C}}\left(100 \mathrm{MHz}, \mathrm{C}_{6} \mathrm{D}_{6}\right) 14.7\left(C\left(3^{\prime}\right)\right), 19.3\left(C\left(2^{\prime}\right)\right), 21.0$ $(C(2)), 21.3(C(8)), 29.3(C(1)), 30.6(C(7)), 33.4(C(6)), 37.0\left(C\left(1^{\prime}\right)\right), 45.8(C(3)), 49.2(C(9)), 59.4(C(5))$, $64.2(C(6 a)), 83.4(C(10 \mathrm{a})) ; m / z\left(\mathrm{ESI}^{+}\right) 209\left([\mathrm{M}+\mathrm{H}]^{+}, 100 \%\right) ; H R M S\left(\mathrm{ESI}^{+}\right) \mathrm{m} / z:[\mathrm{M}+\mathrm{H}]^{+}$Calcd for $\mathrm{C}_{13} \mathrm{H}_{25} \mathrm{~N}_{2}^{+}$209.2012; Found 209.2012.

$(5 S, 6 \mathrm{a} R, 10 \mathrm{a} S)-5$-Pentyldecahydrodipyrrolo[1,2-a:1',2'-c]pyrimidine $[(+)$-tetraponerine 6$] 50$. Palladium black (31 mg, 20\% w/w) was added to a stirred, degassed solution of 46 (155 mg, $0.322 \mathrm{mmol}$, 
$>95: 5$ dr $[(E):(Z)])$ in $\mathrm{MeOH} / \mathrm{H}_{2} \mathrm{O} / \mathrm{AcOH}(10: 1: 1,2 \mathrm{~mL})$. The resultant mixture was stirred under an atmosphere of $\mathrm{H}_{2}(5 \mathrm{~atm})$ at $\mathrm{rt}$ for $48 \mathrm{~h}$. The reaction mixture was then filtered through a short plug of Celite ${ }^{\circledR}$ (eluent $\mathrm{MeOH} / \mathrm{H}_{2} \mathrm{O} / \mathrm{AcOH}, 10: 1: 1$ ) and concentrated in vacuo. The residue was dissolved in $\mathrm{CH}_{2} \mathrm{Cl}_{2}$ $(2 \mathrm{~mL})$, then $\mathrm{K}_{2} \mathrm{CO}_{3}(134 \mathrm{mg}, 0.97 \mathrm{mmol})$ and 4-bromobutanal $(98 \mathrm{mg}, 0.65 \mathrm{mmol})$ were added to the resultant solution. The reaction mixture was stirred at $\mathrm{rt}$ for $2 \mathrm{~h}$, then $\mathrm{H}_{2} \mathrm{O}(2 \mathrm{~mL})$ and satd aq $\mathrm{NaHCO}_{3}(10$ $\mathrm{mL})$ were added. The reaction mixture was then extracted with $\mathrm{CH}_{2} \mathrm{Cl}_{2}(3 \times 5 \mathrm{~mL})$ and the combined organic extracts were dried and concentrated in vacuo. Purification via flash column chromatography (eluent 30-40 ${ }^{\circ} \mathrm{C}$ petrol/ $\left.\mathrm{Me}_{2} \mathrm{CO} / \mathrm{NH}_{4} \mathrm{OH}, 100: 10: 1\right)$ gave (+)-tetraponerine $6(\mathbf{5 0})$ as a pale yellow oil (41 mg, 54\% from 46, >99:1 dr); $[\alpha]_{\mathrm{D}}^{25}+66.3\left(c 1.0\right.$ in $\left.\mathrm{CHCl}_{3}\right) ;\left\{\right.$ lit. ${ }^{4}$ for a sample isolated from the natural source $[\alpha]_{\mathrm{D}}^{20}+35(c$ 0.15 in $\left.\mathrm{CHCl}_{3}\right)$; lit. $^{6}[\alpha]_{\mathrm{D}}^{20}+40\left(c 0.75\right.$ in $\left.\left.\mathrm{CHCl}_{3}\right)\right\} ; v_{\max }(\mathrm{ATR}) 2955,2928,2857,2785(\mathrm{C}-\mathrm{H}){ }^{26} \delta_{\mathrm{H}}(400$ $\left.\mathrm{MHz}, \mathrm{C}_{6} \mathrm{D}_{6}\right) 0.91\left(3 \mathrm{H}, \mathrm{t}, J\right.$ 7.0, C(5') $\left.H_{3}\right), 1.23-1.54\left(11 \mathrm{H}, \mathrm{m}, \mathrm{C}(6) H_{2}, \mathrm{C}(7) H_{\mathrm{A}}, \mathrm{C}(8) H_{\mathrm{A}}, \mathrm{C}\left(1^{\prime}\right) H_{\mathrm{A}}, \mathrm{C}\left(2^{\prime}\right) H_{2}\right.$, $\left.\mathrm{C}\left(3^{\prime}\right) H_{2}, \mathrm{C}\left(4^{\prime}\right) H_{2}\right), 1.54-1.98\left(9 \mathrm{H}, \mathrm{m}, \mathrm{C}(1) H_{2}, \mathrm{C}(2) H_{2}, \mathrm{C}(6 \mathrm{a}) H, \mathrm{C}(7) H_{\mathrm{B}}, \mathrm{C}(8) H_{\mathrm{B}}, \mathrm{C}(9) H_{\mathrm{A}}, \mathrm{C}\left(1^{\prime}\right) H_{\mathrm{B}}\right), 2.31-$ $2.38\left(1 \mathrm{H}, \mathrm{m}, \mathrm{C}(3) H_{\mathrm{A}}\right), 2.39-2.46(1 \mathrm{H}, \mathrm{m}, \mathrm{C}(5) H), 2.87(1 \mathrm{H}$, app t, $J$ 5.3, C(10a)H), $2.94(1 \mathrm{H}$, app td, $J$ 8.5, 2.5, C(9) $\left.H_{\mathrm{B}}\right), 3.03-3.10\left(1 \mathrm{H}, \mathrm{m}, \mathrm{C}(3) H_{\mathrm{B}}\right) ; \delta_{\mathrm{C}}\left(100 \mathrm{MHz}, \mathrm{C}_{6} \mathrm{D}_{6}\right) 14.4\left(C\left(5^{\prime}\right)\right), 21.0(C(2)), 21.3(C(8)), 23.2$ $\left(C\left(4^{\prime}\right)\right), 25.9\left(C\left(2^{\prime}\right)\right), 29.3(C(1)), 30.6(C(7)), 32.7\left(C\left(3^{\prime}\right)\right), 33.5(C(6)), 34.8\left(C\left(1^{\prime}\right)\right), 45.9(C(3)), 49.2(C(9))$, $59.7(C(5)), 64.2(C(6 \mathrm{a})), 83.5(C(10 \mathrm{a})) ; \mathrm{m} / z\left(\mathrm{ESI}^{+}\right) 237\left([\mathrm{M}+\mathrm{H}]^{+}, 100 \%\right) ; \mathrm{HRMS}\left(\mathrm{ESI}^{+}\right) \mathrm{m} / z:[\mathrm{M}+\mathrm{H}]^{+}$ Calcd for $\mathrm{C}_{15} \mathrm{H}_{29} \mathrm{~N}_{2}{ }^{+}$237.2325; Found 237.2326.

$\left(2 R, 2^{\prime} R, \alpha S, \alpha^{\prime} R\right)-1-\left[N\left(1^{\prime}\right)-(\alpha-M e t h y l b e n z y l) p y r r o l i d i n-2 '-y l\right]-2-\left[N-\right.$ benzyl- $N-\left(\alpha^{\prime}-\right.$

methylbenzyl)amino]pent-4-ene 51. DIBAL-H (1.0 M in PhMe, $0.65 \mathrm{~mL}, 0.65 \mathrm{mmol})$ was added dropwise to a stirred solution of $43(200 \mathrm{mg}, 0.380 \mathrm{mmol},>99: 1 \mathrm{dr})$ in $\mathrm{PhMe}(5 \mathrm{~mL})$ at $-78{ }^{\circ} \mathrm{C}$ and the resultant mixture was stirred at $-78{ }^{\circ} \mathrm{C}$ for $1 \mathrm{~h}$. $\mathrm{MeOH}(46 \mu \mathrm{L}, 1.1 \mathrm{mmol})$ and $\mathrm{Ph}_{3} \mathrm{P}=\mathrm{CH}_{2}(0.31 \mathrm{M}$ suspension in $\mathrm{PhMe} / \mathrm{THF}, 6.1 \mathrm{~mL}, 1.9 \mathrm{mmol})^{24}$ were added sequentially, and the resultant mixture was allowed to warm to $\mathrm{rt}$ and stirred at $\mathrm{rt}$ for $16 \mathrm{~h}$, then concentrated in vacuo. The residue was dissolved in $\mathrm{Et}_{2} \mathrm{O}(40 \mathrm{~mL})$ and the resultant solution was filtered through a short plug of $\mathrm{Celite}^{\circledR}$ (eluent $\mathrm{Et}_{2} \mathrm{O}$ ) and concentrated in vacuo. Purification via flash column chromatography (eluent 30-40 ${ }^{\circ} \mathrm{C}$ petrol/Et $\mathrm{E}_{2} \mathrm{O} / \mathrm{NH}_{4} \mathrm{OH}, 100: 20: 1$ ) gave 51 as a colourless oil $(68 \mathrm{mg}, 40 \%,>99: 1 \mathrm{dr})$; $[\alpha]_{\mathrm{D}}^{25}+56.3\left(\right.$ c 1.0 in $\left.\mathrm{CHCl}_{3}\right) ; v_{\max }(\mathrm{ATR}) 3026,2968,2932,2872(\mathrm{C}-$ $\mathrm{H}), 1638,1602,1493,1452(\mathrm{C}=\mathrm{C}) ; \delta_{\mathrm{H}}\left(400 \mathrm{MHz}, \mathrm{CDCl}_{3}\right) 1.31(3 \mathrm{H}, \mathrm{d}, J 6.9, \mathrm{C}(\alpha) M e), 1.43(3 \mathrm{H}, \mathrm{d}, J 6.8$, $\left.\mathrm{C}\left(\alpha^{\prime}\right) M e\right), 1.42-1.48\left(1 \mathrm{H}, \mathrm{m}, \mathrm{C}\left(3^{\prime}\right) H_{\mathrm{A}}\right), 1.52-1.76\left(4 \mathrm{H}, \mathrm{m}, \mathrm{C}(1) H_{\mathrm{A}}, \mathrm{C}\left(3^{\prime}\right) H_{\mathrm{B}}, \mathrm{C}\left(4^{\prime}\right) H_{2}\right), 1.82-1.90(2 \mathrm{H}, \mathrm{m}$, $\left.\mathrm{C}(1) H_{\mathrm{B}}, \mathrm{C}(3) H_{\mathrm{A}}\right), 1.99-2.08\left(1 \mathrm{H}, \mathrm{m}, \mathrm{C}(3) H_{\mathrm{B}}\right), 2.36-2.48\left(2 \mathrm{H}, \mathrm{m}, \mathrm{C}\left(2^{\prime}\right) H, \mathrm{C}\left(5^{\prime}\right) H_{\mathrm{A}}\right), 2.62-2.70(1 \mathrm{H}, \mathrm{m}$, $\mathrm{C}(2) H), 2.81-2.87\left(1 \mathrm{H}, \mathrm{m}, \mathrm{C}\left(5^{\prime}\right) H_{\mathrm{B}}\right), 3.74\left(1 \mathrm{H}, \mathrm{d}, J 14.7, \mathrm{NCH}_{\mathrm{A}} \mathrm{H}_{\mathrm{B}} \mathrm{Ph}\right), 3.79\left(1 \mathrm{H}, \mathrm{d}, J 14.7, \mathrm{NCH}_{\mathrm{A}} H_{\mathrm{B}} \mathrm{Ph}\right)$, $3.83\left(1 \mathrm{H}, \mathrm{q}, J 6.8, \mathrm{C}\left(\alpha^{\prime}\right) H\right), 3.97(1 \mathrm{H}, \mathrm{q}, J 6.9, \mathrm{C}(\alpha) H), 4.80-4.86\left(2 \mathrm{H}, \mathrm{m}, \mathrm{C}(5) H_{2}\right), 5.52(1 \mathrm{H}, \mathrm{ddt}, J 15.8$, 
11.4, 7.0, C(4)H), 7.16-7.35 (13H, m, Ph), 7.39-7.43 (2H, m, Ph); $\delta_{\mathrm{C}}\left(100 \mathrm{MHz}, \mathrm{CDCl}_{3}\right) 17.9(\mathrm{C}(\alpha) M e)$, $21.9\left(\mathrm{C}\left(\alpha^{\prime}\right) M e\right), 22.4\left(C\left(4^{\prime}\right)\right), 30.5\left(C\left(3^{\prime}\right)\right), 36.7(C(3)), 37.6(C(1)), 48.8\left(C\left(5^{\prime}\right)\right), 50.2\left(\mathrm{NCH}_{2} \mathrm{Ph}\right), 55.4(C(2))$, $57.1(C(\alpha)), 58.5\left(C\left(2^{\prime}\right)\right), 59.3\left(C\left(\alpha^{\prime}\right)\right), 115.3(C(5)), 126.6,126.7,127.0(p-P h), 128.0,128.0,128.1,128.2$, 128.3, 128.5 (o,m-Ph), $138.1(C(4)), 142.1,142.3,144.8(i-P h) ; m / z\left(\mathrm{ESI}^{+}\right) 453\left([\mathrm{M}+\mathrm{H}]^{+}, 100 \%\right)$; HRS $\left(\mathrm{ESI}^{+}\right) \mathrm{m} / z:[\mathrm{M}+\mathrm{H}]^{+}$Calcd for $\mathrm{C}_{32} \mathrm{H}_{41} \mathrm{~N}_{2}{ }^{+}$453.3264; Found 453.3259.

\section{$\left(2 R, 2^{\prime} R, \alpha S, \alpha^{\prime} R, Z\right)-1-\left[N\left(1^{\prime}\right)-(\alpha-M e t h y l b e n z y l) p y r r o l i d i n-2 '-y l\right]-2-\left[N-b e n z y l-N-\left(\alpha^{\prime}-\right.\right.$}

methylbenzyl)amino]hept-4-ene 52. DIBAL-H (1.0 M in PhMe, $1.0 \mathrm{~mL}, 1.0 \mathrm{mmol})$ was added dropwise to a stirred solution of $\mathbf{4 3}(242 \mathrm{mg}, 0.459 \mathrm{mmol},>99: 1 \mathrm{dr})$ in $\mathrm{PhMe}(5 \mathrm{~mL})$ at $-78{ }^{\circ} \mathrm{C}$ and the resultant mixture was stirred at $-78{ }^{\circ} \mathrm{C}$ for $1 \mathrm{~h}$. $\mathrm{MeOH}(93 \mu \mathrm{L}, 2.3 \mathrm{mmol})$ and $\mathrm{Ph}_{3} \mathrm{P}=\mathrm{CHCH}_{2} \mathrm{CH}_{3}(0.31 \mathrm{M}$ suspension in $\mathrm{PhMe} / \mathrm{THF}, 14.8 \mathrm{~mL}, 4.59 \mathrm{mmol})^{25}$ were added sequentially, and the resultant mixture was allowed to warm to $\mathrm{rt}$ and stirred at $\mathrm{rt}$ for $16 \mathrm{~h}$, then concentrated in vacuo. The residue was dissolved in $\mathrm{Et}_{2} \mathrm{O}(40 \mathrm{~mL})$ and the resultant solution was filtered through a short plug of Celite ${ }^{\circledR}$ (eluent $\mathrm{Et}_{2} \mathrm{O}$ ) and concentrated in vacuo. Purification via flash column chromatography (eluent 30-40 ${ }^{\circ} \mathrm{C}$ petrol/ $\mathrm{Et}_{2} \mathrm{O} / \mathrm{NH}_{4} \mathrm{OH}, 100: 20: 1$ ) gave 52 as a pale yellow oil (106 mg, 48\%, >95:5 dr [(Z):(E)]); $[\alpha]_{\mathrm{D}}^{25}+53.9\left(c 1.0\right.$ in $\left.\mathrm{CHCl}_{3}\right) ; v_{\max }$ (ATR) 3025, 2964, 2932, $2872(\mathrm{C}-\mathrm{H}), 1602,1493,1453(\mathrm{C}=\mathrm{C}) ; \delta_{\mathrm{H}}\left(400 \mathrm{MHz}, \mathrm{CDCl}_{3}\right) 0.86(3 \mathrm{H}, \mathrm{t}, J$ 7.5, C(7)H $), 1.32(3 \mathrm{H}, \mathrm{d}$, $J$ 6.8, C( $\alpha) M e), 1.41-1.47\left(1 \mathrm{H}, \mathrm{m}, \mathrm{C}(1) H_{\mathrm{A}}\right), 1.44\left(3 \mathrm{H}, \mathrm{d}, J\right.$ 6.8, C $\left.\left(\alpha^{\prime}\right) M e\right), 1.53-1.77\left(4 \mathrm{H}, \mathrm{m}, \mathrm{C}(1) H_{\mathrm{B}}\right.$, $\left.\mathrm{C}\left(3^{\prime}\right) H_{\mathrm{A}}, \mathrm{C}\left(4^{\prime}\right) H_{2}\right), 1.79-1.92\left(4 \mathrm{H}, \mathrm{m}, \mathrm{C}(3) H_{\mathrm{A}}, \mathrm{C}(6) H_{2}, \mathrm{C}\left(3^{\prime}\right) H_{\mathrm{B}}\right), 1.93-2.03\left(1 \mathrm{H}, \mathrm{m}, \mathrm{C}(3) H_{\mathrm{B}}\right), 2.36-2.45(2 \mathrm{H}$, m, C(2')H, C(4') $\left.H_{\mathrm{A}}\right), 2.58-2.66(1 \mathrm{H}, \mathrm{m}, \mathrm{C}(2) H), 2.84\left(1 \mathrm{H}, \mathrm{ddd}, J 9.1,7.8,2.4, \mathrm{C}\left(4^{\prime}\right) H_{\mathrm{B}}\right), 3.73(1 \mathrm{H}, \mathrm{d}, J 14.8$, $\left.\mathrm{NCH}_{\mathrm{A}} \mathrm{H}_{\mathrm{B}} \mathrm{Ph}\right), 3.77\left(1 \mathrm{H}, \mathrm{d}, J 14.8, \mathrm{NCH}_{\mathrm{A}} H_{\mathrm{B}} \mathrm{Ph}\right), 3.85\left(1 \mathrm{H}, \mathrm{q}, J\right.$ 6.8, $\left.\mathrm{C}\left(\alpha^{\prime}\right) H\right), 3.99(1 \mathrm{H}, \mathrm{q}, J$ 6.8, $\mathrm{C}(\alpha) H), 5.09$ $(1 \mathrm{H}$, app dtt, $J 10.8,6.8,1.4, \mathrm{C}(4) H), 5.23(1 \mathrm{H}$, app dtt, $J 10.8,7.1,1.6, \mathrm{C}(5) H), 7.15-7.41(15 \mathrm{H}, \mathrm{m}, P h) ; \delta_{\mathrm{C}}$ $\left(100 \mathrm{MHz}, \mathrm{CDCl}_{3}\right) 14.2(C(7)), 18.0(\mathrm{C}(\alpha) M e), 20.9(C(6)), 21.9\left(\mathrm{C}\left(\alpha^{\prime}\right) M e\right), 22.4\left(C\left(4^{\prime}\right)\right), 29.7(C(3)), 30.6$ $(C(1)), 37.4\left(C\left(3^{\prime}\right)\right), 48.7\left(C\left(5^{\prime}\right)\right), 50.1\left(\mathrm{NCH}_{2} \mathrm{Ph}\right), 55.9(C(2)), 57.0(C(\alpha)), 58.6\left(C\left(2^{\prime}\right)\right), 59.2\left(C\left(\alpha^{\prime}\right)\right)$, 126.6, 126.6, $127.0(p-P h), 128.0,128.1(o, m-P h), 128.2(C(4)), 128.2,128.2,128.3,128.6(o, m-P h), 131.7(C(5))$, 142.2, 142.3, $145.0(i-P h) ; m / z\left(\mathrm{ESI}^{+}\right) 481\left([\mathrm{M}+\mathrm{H}]^{+}, 100 \%\right) ; \operatorname{HRMS}\left(\mathrm{ESI}^{+}\right) \mathrm{m} / z:[\mathrm{M}+\mathrm{H}]^{+} \mathrm{Calcd}$ for $\mathrm{C}_{34} \mathrm{H}_{45} \mathrm{~N}_{2}^{+} 481.3577$; Found 481.3571 .

$(5 R, 6 a R, 10 a S)-5-P r o p y l d e c a h y d r o d i p y r r o l o\left[1,2-a: 1^{\prime}, 2^{\prime}-c\right]$ pyrimidine [(+)-tetraponerine 1$] 55$. Palladium black (29 mg, 20\% w/w) was added to a stirred, degassed solution of 51 (145 mg, $0.320 \mathrm{mmol}$, >99:1 dr) in $\mathrm{MeOH} / \mathrm{H}_{2} \mathrm{O} / \mathrm{AcOH}(10: 1: 1,2 \mathrm{~mL})$. The resultant mixture was stirred under an atmosphere of $\mathrm{H}_{2}$ $(5 \mathrm{~atm})$ at $\mathrm{rt}$ for $48 \mathrm{~h}$. The reaction mixture was then filtered through a short plug of Celite ${ }^{\circledR}$ (eluent $\left.\mathrm{MeOH} / \mathrm{H}_{2} \mathrm{O} / \mathrm{AcOH}, 10: 1: 1\right)$ and concentrated in vacuo. The residue was dissolved in $\mathrm{CH}_{2} \mathrm{Cl}_{2}(2 \mathrm{~mL})$, then $\mathrm{K}_{2} \mathrm{CO}_{3}$ (133 mg, $0.96 \mathrm{mmol}$ ) and 4-bromobutanal (96 mg, $0.64 \mathrm{mmol}$ ) were added to the resultant solution. 
The reaction mixture was stirred at $\mathrm{rt}$ for $2 \mathrm{~h}$, then $\mathrm{H}_{2} \mathrm{O}(2 \mathrm{~mL})$ and satd aq $\mathrm{NaHCO}_{3}(10 \mathrm{~mL})$ were added. The reaction mixture was then extracted with $\mathrm{CH}_{2} \mathrm{Cl}_{2}(3 \times 5 \mathrm{~mL})$ and the combined organic extracts were dried and concentrated in vacuo. Purification via flash column chromatography (eluent $30-40{ }^{\circ} \mathrm{C}$ petrol/ $\left.\mathrm{Me}_{2} \mathrm{CO} / \mathrm{NH}_{4} \mathrm{OH}, 100: 10: 1\right)$ gave (+)-tetraponerine 1 (55) as a colourless oil $(10 \mathrm{mg}$, 15\% from 51, >99: $1 \mathrm{dr}) ;[\alpha]_{\mathrm{D}}^{25}+14.4\left(c 0.13\right.$ in $\left.\mathrm{CHCl}_{3}\right) ;\left\{\right.$ lit. $^{5}[\alpha]_{\mathrm{D}}^{20}+11\left(c 0.14\right.$ in $\left.\mathrm{CHCl}_{3}\right)$; lit. ${ }^{6}[\alpha]_{\mathrm{D}}^{20}+14(c 0.498$ in $\left.\left.\mathrm{CHCl}_{3}\right)\right\} ; v_{\max }(\mathrm{ATR}) 2927,2801(\mathrm{C}-\mathrm{H}) ;{ }^{26} \delta_{\mathrm{H}}\left(400 \mathrm{MHz}, \mathrm{C}_{6} \mathrm{D}_{6}\right) 0.96\left(3 \mathrm{H}, \mathrm{t}, J 7.3, \mathrm{C}\left(3^{\prime}\right) H_{3}\right), 1.23-1.45(6 \mathrm{H}$, m, C $\left.(1) H_{\mathrm{A}}, \mathrm{C}(6) H_{\mathrm{A}}, \mathrm{C}(7) H_{\mathrm{A}}, \mathrm{C}(8) H_{\mathrm{A}}, \mathrm{C}\left(1^{\prime}\right) H_{\mathrm{A}}, \mathrm{C}\left(2^{\prime}\right) H_{\mathrm{A}}\right), 1.49-1.69\left(3 \mathrm{H}, \mathrm{m}, \mathrm{C}(7) H_{\mathrm{B}}, \mathrm{C}(8) H_{\mathrm{B}}, \mathrm{C}\left(2^{\prime}\right) H_{\mathrm{B}}\right)$, 1.69-1.91 (6H, m, C(1) $\left.H_{\mathrm{B}}, \mathrm{C}(2) H_{2}, \mathrm{C}(6) H_{\mathrm{B}}, \mathrm{C}(9) H_{\mathrm{A}}, \mathrm{C}\left(1^{\prime}\right) H_{\mathrm{B}}\right), 1.91-2.02(1 \mathrm{H}, \mathrm{m}, \mathrm{C}(6 \mathrm{a}) H), 2.82-2.91(3 \mathrm{H}$, m, C(3) $\left.H_{\mathrm{A}}, \mathrm{C}(5) H, \mathrm{C}(9) H_{\mathrm{B}}\right), 3.21\left(1 \mathrm{H}\right.$, app q, $J$ 7.7, C(3)H $\left.H_{\mathrm{B}}\right), 3.48$, (1H, app t, $\left.J 2.3, \mathrm{C}(10 \mathrm{a}) H\right)$; $\delta_{\mathrm{H}}(400$ $\left.\mathrm{MHz}, \mathrm{CDCl}_{3}\right) 0.90\left(3 \mathrm{H}, \mathrm{t}, J\right.$ 7.3, C(3') $\left.H_{3}\right), 1.24-1.43\left(3 \mathrm{H}, \mathrm{m}, \mathrm{C}(7) H_{\mathrm{A}}, \mathrm{C}\left(2^{\prime}\right) H_{2}\right), 1.43-1.96\left(12 \mathrm{H}, \mathrm{m}, \mathrm{C}(1) H_{2}\right.$, $\left.\mathrm{C}(2) H_{2}, \mathrm{C}(6) H_{2}, \mathrm{C}(7) H_{\mathrm{B}}, \mathrm{C}(8) H_{2}, \mathrm{C}(9) H_{\mathrm{A}}, \mathrm{C}\left(1^{\prime}\right) H_{2}\right), 2.00-2.10(1 \mathrm{H}, \mathrm{m}, \mathrm{C}(6 \mathrm{a}) H), 2.82(1 \mathrm{H}, \mathrm{td}, J$ 7.9, 3.0, $\left.\mathrm{C}(3) H_{\mathrm{A}}\right), 2.91-3.01\left(2 \mathrm{H}, \mathrm{m}, \mathrm{C}(5) H, \mathrm{C}(9) H_{\mathrm{B}}\right), 3.08\left(1 \mathrm{H}\right.$, app q, $\left.J 7.9, \mathrm{C}(3) H_{\mathrm{B}}\right), 3.39(1 \mathrm{H}$, app d, $J 3.7$, $\mathrm{C}(10 \mathrm{a}) H) ; \delta_{\mathrm{C}}\left(100 \mathrm{MHz}, \mathrm{C}_{6} \mathrm{D}_{6}\right) 14.5\left(C\left(3^{\prime}\right)\right), 20.4(C(8)), 20.8\left(C\left(2^{\prime}\right)\right), 22.0(C(2)), 30.1,30.1(C(1), C(6))$, $31.6(C(7)), 35.4\left(C\left(1^{\prime}\right)\right), 50.0(C(9)), 51.0(C(3)), 53.7(C(5)), 58.3(C(6 \mathrm{a})), 76.5(C(10 \mathrm{a}))$; $\delta_{\mathrm{C}}(100 \mathrm{MHz}$, $\left.\mathrm{CDCl}_{3}\right) 14.3\left(C\left(3^{\prime}\right)\right), 20.1(C(8)), 20.6\left(C\left(2^{\prime}\right)\right), 21.3(C(2)), 29.4(C(6)), 29.7(C(1)), 31.0(C(7)), 34.0\left(C\left(1^{\prime}\right)\right)$, $50.1(C(9)), 50.3(C(3)), 53.6(C(5)), 58.3(C(6 \mathrm{a})), 76.9(C(10 \mathrm{a})),{ }^{27} \mathrm{~m} / \mathrm{z}\left(\mathrm{ESI}^{+}\right) 209\left([\mathrm{M}+\mathrm{H}]^{+}, 100 \%\right)$; HRMS $\left(\mathrm{ESI}^{+}\right) \mathrm{m} / z:[\mathrm{M}+\mathrm{H}]^{+}$Calcd for $\mathrm{C}_{13} \mathrm{H}_{25} \mathrm{~N}_{2}{ }^{+}$209.2012; Found 209.2013.

$(5 R, 6 a R, 10 \mathrm{a} S)-5-P e n t y l d e c a h y d r o d i p y r r o l o\left[1,2-a: 1^{\prime}, 2^{\prime}-c\right]$ pyrimidine [(+)-tetraponerine 5$] 56$. Palladium black (31 mg, 20\% w/w) was added to a stirred, degassed solution of $\mathbf{5 2}(154 \mathrm{mg}, 0.330 \mathrm{mmol})$ in $\mathrm{MeOH} / \mathrm{H}_{2} \mathrm{O} / \mathrm{AcOH}(10: 1: 1,2 \mathrm{~mL})$. The resultant mixture was stirred under an atmosphere of $\mathrm{H}_{2}(5 \mathrm{~atm})$ at $\mathrm{rt}$ for $48 \mathrm{~h}$. The reaction mixture was then filtered through a short plug of Celite ${ }^{\circledR}$ (eluent $\mathrm{MeOH} / \mathrm{H}_{2} \mathrm{O} / \mathrm{AcOH}$, 10:1:1) and concentrated in vacuo. The residue was dissolved in $\mathrm{CH}_{2} \mathrm{Cl}_{2}(2 \mathrm{~mL})$, then $\mathrm{K}_{2} \mathrm{CO}_{3}(137 \mathrm{mg}, 0.99$ $\mathrm{mmol})$ and 4-bromobutanal $(99 \mathrm{mg}, 0.66 \mathrm{mmol})$ were added to the resultant solution. The reaction mixture was stirred at $\mathrm{rt}$ for $2 \mathrm{~h}$, then $\mathrm{H}_{2} \mathrm{O}(2 \mathrm{~mL})$ and satd aq $\mathrm{NaHCO}_{3}(10 \mathrm{~mL})$ were added. The reaction mixture was then extracted with $\mathrm{CH}_{2} \mathrm{Cl}_{2}(3 \times 5 \mathrm{~mL})$ and the combined organic extracts were dried and concentrated in vacuo. Purification via flash column chromatography (eluent $30-40{ }^{\circ} \mathrm{C}$ petrol/ $\mathrm{Me}_{2} \mathrm{CO} / \mathrm{NH}_{4} \mathrm{OH}, 100: 10: 1$ ) gave (+)-tetraponerine $5(\mathbf{5 6})$ as a colourless oil $(11 \mathrm{mg}, 14 \%$ from $\mathbf{5 2},>99: 1 \mathrm{dr})$; $[\alpha]_{\mathrm{D}}^{25}+12.4(c 0.13$ in $\left.\mathrm{CHCl}_{3}\right) ;\left\{\right.$ lit. ${ }^{4}$ for a sample isolated from the natural source $[\alpha]_{\mathrm{D}}^{20}+10\left(c 0.2\right.$ in $\left.\mathrm{CHCl}_{3}\right) ;$ lit. $^{5}[\alpha]_{\mathrm{D}}^{20}+10(c$ 0.24 in $\left.\mathrm{CHCl}_{3}\right)$; lit. ${ }^{6}[\alpha]_{\mathrm{D}}^{20}+14\left(c 1.6\right.$ in $\left.\left.\mathrm{CHCl}_{3}\right)\right\} ; v_{\max }(\mathrm{ATR}) 2980,2930,2794(\mathrm{C}-\mathrm{H}){ }^{26} \delta_{\mathrm{H}}(400 \mathrm{MHz}$, $\left.\mathrm{C}_{6} \mathrm{D}_{6}\right) 0.93\left(3 \mathrm{H}, \mathrm{t}, J 6.8, \mathrm{C}\left(5^{\prime}\right) H_{3}\right), 1.28-1.46\left(10 \mathrm{H}, \mathrm{m}, \mathrm{C}(1) H_{\mathrm{A}}, \mathrm{C}(6) H_{\mathrm{A}}, \mathrm{C}(7) H_{\mathrm{A}}, \mathrm{C}(8) H_{\mathrm{A}}, \mathrm{C}\left(1^{\prime}\right) H_{\mathrm{A}}, \mathrm{C}\left(2^{\prime}\right) H_{\mathrm{A}}\right.$, $\left.\mathrm{C}\left(3^{\prime}\right) H_{2}, \mathrm{C}\left(4^{\prime}\right) H_{2}\right), 1.50-1.69\left(3 \mathrm{H}, \mathrm{m}, \mathrm{C}(7) H_{\mathrm{B}}, \mathrm{C}(8) H_{\mathrm{B}}, \mathrm{C}\left(2^{\prime}\right) H_{\mathrm{B}}\right), 1.69-1.92\left(6 \mathrm{H}, \mathrm{m}, \mathrm{C}(1) H_{\mathrm{B}}, \mathrm{C}(2) H_{2}\right.$, 
$\left.\mathrm{C}(6) H_{\mathrm{B}}, \mathrm{C}(9) H_{\mathrm{A}}, \mathrm{C}\left(1^{\prime}\right) H_{\mathrm{B}}\right), 1.94-2.01(1 \mathrm{H}, \mathrm{m}, \mathrm{C}(6 \mathrm{a}) H), 2.83-2.92\left(3 \mathrm{H}, \mathrm{m}, \mathrm{C}(3) H_{\mathrm{A}}, \mathrm{C}(5) H, \mathrm{C}(9) H_{\mathrm{B}}\right), 3.23$ $\left(1 \mathrm{H}\right.$, app q, $J$ 7.8, C(3) $\left.H_{\mathrm{B}}\right), 3.51(1 \mathrm{H}$, app t, $J 2.1, \mathrm{C}(10 \mathrm{a}) H) ; \delta_{\mathrm{H}}\left(400 \mathrm{MHz}, \mathrm{CDCl}_{3}\right) 0.88(3 \mathrm{H}, \mathrm{t}, J 6.8$, $\left.\mathrm{C}\left(5^{\prime}\right) H_{3}\right), 1.22-1.42\left(7 \mathrm{H}, \mathrm{m}, \mathrm{C}(7) H_{\mathrm{A}}, \mathrm{C}\left(2^{\prime}\right) H_{2}, \mathrm{C}\left(3^{\prime}\right) H_{2}, \mathrm{C}\left(4^{\prime}\right) H_{2}\right), 1.43-1.96\left(12 \mathrm{H}, \mathrm{m}, \mathrm{C}(1) H_{2}, \mathrm{C}(2) H_{2}\right.$, $\left.\mathrm{C}(6) H_{2}, \mathrm{C}(7) H_{\mathrm{B}}, \mathrm{C}(8) H_{2}, \mathrm{C}(9) H_{\mathrm{A}}, \mathrm{C}\left(1^{\prime}\right) H_{2}\right), 2.01-2.10(1 \mathrm{H}, \mathrm{m}, \mathrm{C}(6 \mathrm{a}) H), 2.82(1 \mathrm{H}$, app td, $J$ 8.0, 2.8, $\left.\mathrm{C}(3) H_{\mathrm{A}}\right), 2.89-3.01\left(2 \mathrm{H}, \mathrm{m}, \mathrm{C}(5) H, \mathrm{C}(9) H_{\mathrm{B}}\right), 3.08\left(1 \mathrm{H}\right.$, app q, $J$ 8.0, C(3)H $\left.H_{\mathrm{B}}\right), 3.39(1 \mathrm{H}$, app d, $J 3.5$, $\mathrm{C}(10 \mathrm{a}) H) ; \delta_{\mathrm{C}}\left(100 \mathrm{MHz}, \mathrm{C}_{6} \mathrm{D}_{6}\right) 14.4\left(C\left(5^{\prime}\right)\right), 20.4(C(8)), 22.0(C(2)), 23.2\left(C\left(4^{\prime}\right)\right), 27.5\left(C\left(2^{\prime}\right)\right), 30.1,30.2$ $(C(1), C(6)), 31.6(C(7)), 32.5\left(C\left(3^{\prime}\right)\right), 33.2\left(C\left(1^{\prime}\right)\right), 50.0(C(9)), 51.1(C(3)), 54.1(C(5)), 58.3(C(6 a)), 76.5$ $(C(10 \mathrm{a})) ; \delta_{\mathrm{C}}\left(100 \mathrm{MHz}, \mathrm{CDCl}_{3}\right) 14.3\left(C\left(5^{\prime}\right)\right), 20.2(C(8)), 21.3(C(2)), 22.8\left(C\left(4^{\prime}\right)\right), 27.2\left(C\left(2^{\prime}\right)\right), 29.4(C(6))$, $29.7(C(1)), 31.0(C(7)), 31.7\left(C\left(1^{\prime}\right)\right), 32.1\left(C\left(3^{\prime}\right)\right), 50.0(C(9)), 50.3(C(3)), 53.9(C(5)), 58.3(C(6 a)), 76.8$ $(C(10 \mathrm{a})){ }^{27} \mathrm{~m} / z\left(\mathrm{ESI}^{+}\right) 237\left([\mathrm{M}+\mathrm{H}]^{+}, 100 \%\right) ; \operatorname{HRMS}\left(\mathrm{ESI}^{+}\right) \mathrm{m} / z:[\mathrm{M}+\mathrm{H}]^{+}$Calcd for $\mathrm{C}_{15} \mathrm{H}_{29} \mathrm{~N}_{2}{ }^{+} 237.2325$; Found 237.2324.

\section{ASSOCIATED CONTENT}

Supporting Information Available: Copies of ${ }^{1} \mathrm{H}$ and ${ }^{13} \mathrm{C}$ NMR spectra. This material is available free of charge via the Internet at http://pubs.acs.org.

\section{AUTHOR INFORMATION}

\section{Corresponding Author}

*E-mail: steve.davies@chem.ox.ac.uk.

\section{Notes}

The authors declare no competing financial interest.

\section{References and Notes}

${ }^{1}$ Brakman, J. C.; Daloze, D.; Pasteels, J. M.; Van Hecke, P.; Declercq, J. M.; Stinwell, V.; Francke W. Z. Naturforsch. 1987, 42c, 627.

${ }^{2}$ Kem, W. R.; Wildeboer, K.; LeFrancois, S.; Raja, M.; Marszalec, W.; Braekman, J.-C. Cell. Mol. Neurobiol. 2004, 24, 535.

${ }^{3}$ Yue, C.; Royer, J.; Husson, H.-P. J. Org. Chem. 1990, 55, 1140.

${ }^{4}$ Macours, P.; Braekman, J.-C.; Daloze, D. Tetrahedron 1995, 51, 1415.

${ }^{5}$ Yue, C.; Gauthier, I.; Royer, J.; Husson, H.-P. J. Org. Chem. 1996, 61, 4949. 
${ }^{6}$ Bosque, I.; Gonzalez-Gomez, J. C.; Loza, M. I.; Brea, J. J. Org. Chem. 2014, 79, 3982.

${ }^{7}$ Merlin, P.; Braekman, J.-C.; Daloze, D.; Pasteels, J. M. J. Chem. Ecol. 1988, 14, 517.

8 (a) Merlin, P.; Braekman, J.-C.; Daloze, D. Tetrahedron Lett. 1988, 29, 1691. (b) Jones, T. H. Tetrahedron Lett. 1990, 31, 1535. (c) Jones, T. H. Tetrahedron Lett. 1990, 31, 4543. (d) Merlin, P.; Braekman, J.-C.; Daloze, D. Tetrahedron 1991, 47, 3805. (e) Barluenga, J.; Tomás, M.; Kouznetsov, V.; Rubio, E. J. Org. Chem. 1994, 59, 3699. (f) Devijver, C.; Macours, P.; Braekman, J.-C.; Daloze, D.; Pasteels, J. M. Tetrahedron 1995, 51, 10913. (g) Kim, J. T.; Gevorgyan, V. Org. Lett. 2002, 4, 4697. (h) Kim, J. T.; Butt, J.; Gevorgyan, V. J. Org. Chem. 2004, 69, 5638. (i) Charette, A. B.; Mathieu, S.; Martel, J. Org. Lett. 2005, 7, 5401.

${ }^{9}$ (a) Takahata, H.; Kubota, M.; Ikota, N. J. Org. Chem. 1999, 64, 8594. (b) Stragies, R.; Blechert, S. J. Am. Chem. Soc. 2000, 122, 9584. (c) Airiau, E.; Girard, N.; Pizzeti, M.; Salvadori, J.; Taddei, M.; Mann, A. J. Org. Chem. 2010, 75, 8670. (d) Bosque, I.; González-Gómez, J. C.; Guijarro, A.; Foubelo, F.; Yus, M. J. Org. Chem. 2012, 77, 10340. (e) Strassnig, I.; Körber, K.; Hünger, U.; Kunz, H. Synthesis 2015, 47, 2299. (f) Pacheco, J. C. O.; Lipp, A.; Nauth, A. M.; Acke, F.; Dietz, J.-P.; Opatz, T. Chem. Eur. J. 2016, 22, 5409. ${ }^{10}$ Several formal syntheses of the tetraponerine alkaloids have also been reported; see: (a) Plehiers, M.; Heilporn, S.; Ekelmans, D.; Leclercq, S.; Sangermano, M.; Braekman, J.-C.; Daloze, D. Can. J. Chem. 2000, 78, 1030. (b) Bisai, A.; Singh, V. K. Tetrahedron Lett. 2007, 48, 1907. (c) Liu, J.-D.; Chen, Y.-C.; Zhang, G.-B.; Li, Z.-Q.; Chen, P.; Du, J.-Y.; Tu, Y.-Q.; Fan, C.-A. Adv. Synth. Catal. 2011, 353, 2721.

${ }^{11}$ Various structural analogues of the tetraponerine alkaloids have also been prepared; see: (a) Zhu, Z; Quirion, J.-C.; Husson, H.-P. Tetrahedron Lett. 1989, 30, 5137. (b) Rouchaud, A.; Braekman, J.-C. Eur. J. Org. Chem. 2009, 2666. See also: Ref 8 h.

${ }^{12}$ For a recent review of this methodology, see: Davies, S. G.; Fletcher, A. M.; Roberts, P. M.; Thomson, J. E. Tetrahedron: Asymmetry 2012, 23, 1111.

${ }^{13}$ Davies, S. G.; Fletcher, A. M.; Roberts, P. M.; Smith, A. D. Tetrahedron 2008, 65, 10192.

${ }^{14}$ Csatayová, K.; Davies, S. G.; Fletcher, A. M.; Ford, J. G.; Klauber, D. J.; Roberts, P. M.; Thomson, J. E. J. Org. Chem. 2014, 79, 10932.

15 (a) Davies, S. G.; Iwamoto, K.; Smethurst, C. A. P.; Smith, A. D.; Rodríguez-Solla, H. Synlett 2002, 1146. (b) Chippindale, A. M.; Davies, S. G.; Iwamoto, K.; Parkin, R. M.; Smethurst, C. A. P.; Smith, A. D.; Rodríguez-Solla, H. Tetrahedron 2003, 59, 3253. (c) Csatayová, K.; Davies, S. G.; Figuccia, A. L. A.; Fletcher, A. M.; Ford, J. G.; Lee, J. A.; Roberts, P. M.; Saward, B. G.; Song, H.; Thomson, J. E. Tetrahedron 2015, 71, 9131. 
${ }^{16}$ tert-Butyl sorbate 3 was prepared in $91 \%$ yield upon esterification of commercially available sorbic acid with isobutene; see: Ref 14.

${ }^{17}$ For example, see: (a) Davies, S. G.; Durbin, M. J.; Goddard, E. C.; Kelly, P. M.; Kurosawa, W.; Lee, J. A.; Nicholson, R. L.; Price, P. D.; Roberts, P. M.; Russell, A. J.; Scott, P. M.; Smith, A. D. Org. Biomol. Chem. 2009, 7, 761. (b) Davies, S. G.; Lee, J. A.; Roberts, P. M.; Thomson, J. E.; Yin, J. Tetrahedron 2011, $67,6382$.

${ }^{18}$ For example, see: (a) Magano, J.; Bowles, D.; Conway, B.; Nanninga, T. N.; Winkle, D. D. Tetrahedron Lett. 2009, 50, 6325. (b) Davies, S. G.; Ichihara, O.; Roberts, P. M.; Thomson, J. E. Tetrahedron 2011, 67, 216. (c) Davies, S. G.; Lee, J. A.; Roberts, P. M.; Stonehouse, J. P.; Thomson, J. E. J. Org. Chem. 2012, 77, 9724.

${ }^{19}$ The application of an alternative olefination procedure gave a 75:25 $\mathrm{dr}[(Z):(E)]$ mixture of olefins, which was analysed by ${ }^{13} \mathrm{C}$ NMR spectroscopy. The configuration of the double bond within $(Z)-\mathbf{2 4}$ was then assigned from the diagnostic differences in chemical shift for the allylic carbon atoms within the two diastereoisomeric olefin products: for the $(E)$-diastereoisomer $\delta_{\mathrm{C}}=35.4(C(3)), 25.8(C(6))$ and for the $(\mathrm{Z})$ diatereoisomer $\delta_{\mathrm{C}}=29.6(C(3)), 20.9(C(6))$. (Z)-Olefins typically appear $\sim 6 \mathrm{ppm}$ lower in their ${ }^{13} \mathrm{C}$ NMR spectra than the corresponding $(E)$-diastereoisomers; for a discussion of this " $\gamma$-steric effect" in the ${ }^{13} \mathrm{C}$ NMR spectra of cis- and trans-olefins, see: Jacobsen, N. E. In NMR Data Interpretation Explained: Understanding $1 D$ and 2D NMR Spectra of Organic Compounds and Natural Products; Wiley, Hoboken, New Jersey, USA, 2017; pp 212.

${ }^{20}$ The $(Z)$-configurations of the $\mathrm{C}=\mathrm{C}$ double bonds within $\mathbf{3 0 , 4 6}$ and $\mathbf{5 2}$ were assigned by analogy to that within $(Z)$-24 in the absence of authentic samples of the corresponding $(E)$-diastereoisomers.

${ }^{21}$ Oxidation of ent-36 to give the corresponding pyrrole was previously noted upon attempted Pd-mediated tandem hydrogenation/hydrogenolysis; see: Ref 15 a.

${ }^{22}$ As (+)-tetraponerine $2(49)$ and (+)-tetraponerine $6(50)$ were derived from the common precursor $(R, R, R, R)-\mathbf{4 2}$, the configurations of $\mathbf{4 6}, \mathbf{4 8}$ and $\mathbf{5 0}$ remain secure.

${ }^{23}$ Pangborn, A. B.; Giardello, M. A.; Grubbs, R. H.; Rosen, R. K.; Timmers, F. J. Organometallics 1996, 15, 1518.

${ }^{24}$ The solution of $\mathrm{Ph}_{3} \mathrm{P}=\mathrm{CH}_{2}$ was prepared on the requisite scale by the addition of KHMDS $(0.5 \mathrm{M}$ in $\mathrm{PhMe}, 1.0$ equiv) to stirred suspension of methyltriphenylphosponium iodide (1.0 $\mathrm{M}$ in THF, 1.0 equiv) at $\mathrm{rt}$ and the resultant suspension was stirred at $\mathrm{rt}$ for $1 \mathrm{~h}$. 
${ }^{25}$ The solution of $\mathrm{Ph}_{3} \mathrm{P}=\mathrm{CHCH}_{2} \mathrm{CH}_{3}$ was prepared on the requisite scale by the addition of KHMDS $(0.5 \mathrm{M}$ in PhMe, 1.0 equiv) to stirred suspension of propyltriphenylphosponium bromide (1.0 M in THF, 1.0 equiv) at $\mathrm{rt}$ and the resultant suspension was stirred at $\mathrm{rt}$ for $1 \mathrm{~h}$.

26 The Bolmann band frequencies and intensities in the IR spectra of the tetraponerine alkaloids have previously been used to assign the configuration at the $\mathrm{C}(5)$-position. The even numbered alkaloids (i.e., T2, T4, T6 and T8) typically have more intense Bohlmann bands at $\sim 2790 \mathrm{~cm}^{-1}$, whereas the odd numbered alkaloids (i.e., T1, T3, T5 and T7) display weaker Bohlmann bands at $\sim 2805 \mathrm{~cm}^{-1}$; for further discussion of this analysis, see: Garraffo, H. M.; Spande, T. F.; Jain, P.; Kaneko, T.; Jones, T. H.; Blum, M. S.; Ali, T. M. M.; Snelling, R. R.; Isbell, L. A.; Robertson, H. G.; Daly, J. W. Rapid Commun. Mass Spectrom. 2001, 15, 1409.

${ }^{27}$ For both (+)-tetraponerine $1(\mathbf{5 5})$ and (+)-tetraponerine $5(\mathbf{5 6})$, the resonance corresponding to $C(10 \mathrm{a})$ in their ${ }^{13} \mathrm{C}$ NMR spectra (in $\mathrm{CDCl}_{3}$ ) was obscured by the solvent peak. 